\title{
Stability analysis of stationary light transmission in nonlinear photonic structures
}

\author{
Dmitry E. Pelinovsky ${ }^{\dagger}$, and Arnd Scheel ${ }^{\dagger \dagger}$ \\ $\dagger$ Department of Mathematics, McMaster University, \\ 1280 Main Street West, Hamilton, Ontario, Canada, L8S 4K1 \\ †† School of Mathematics, University of Minnesota, \\ 206 Church Street, S.E., Minneapolis, MN 55455, USA
}

February 24, 2003

\begin{abstract}
We study optical bistability of stationary light transmission in nonlinear periodic structures of finite and semi-infinite length. For finite-length structures, the system exhibits instability mechanisms typical for dissipative dynamical systems. We construct a LeraySchauder stability index and show that it equals the sign of the Evans function in $\lambda=0$. As a consequence, stationary solutions with negative-slope transmission function are always unstable. In semi-infinite structures, the system may have stationary localized solutions with non-monotonically decreasing amplitudes. We show that the localized solution with a positive-slope amplitude at the input is always unstable. We also derive expansions for finite size effects and show that the bifurcation diagram stabilizes in the limit of the infinite domain size.
\end{abstract}

\section{Introduction}

This paper addresses optical bistability in nonlinear periodic structures of finite and semiinfinite length, referred to as the photonic gratings. Photonic gratings can be fabricated with a periodical concatenation of optical layers of different linear and nonlinear refractive indices. When these structures are illuminated with incident light, a sequence of frequency intervals in the photonic band spectrum are prohibited. They are referred to as the photonic band gaps [9] and center at frequencies of parametric resonance between the light waves and the periodic structure.

The first band gap is called the Bragg resonance gap. It corresponds to a light wavelength matching the double period of the structure. Light waves with frequencies in the Bragg 
resonance gap are strongly reflected, but light transmission is still possible in finite length structures. Light transmission is generally intensity-dependent in nonlinear photonic gratings, such that transmission of light waves of small intensities is typically observed in a stable stationary regime, but light transmission might undergo instabilities and bifurcations for larger incident intensities.

The light transmission in the first Bragg resonance gap is modeled by coupled-mode equations for the complex amplitudes of incident and reflected light. The equations can be derived as a coupled-mode approximation to the spatially one-dimensional, time-dependent Maxwell equations for the electric field of light waves, with nonlinear refractive index, $n=n\left(z,|E|^{2}\right)$. In this framework, the light waves are decomposed into forward $(A)$ and backward $(B)$ waves $[6,20]$

$$
E(z, t)=A(z, t) \mathrm{e}^{\mathrm{i}\left(k_{0} z-\omega_{0} t\right)}+B(z, t) \mathrm{e}^{-\mathrm{i}\left(k_{0} z+\omega_{0} t\right)}+\text { higher-order Fourier terms. }
$$

To leading order, the time-evolution of the complex amplitudes $A$ and $B$ is governed by a system of semilinear, hyperbolic equations of the general form

$$
\begin{aligned}
\mathrm{i}\left(\frac{\partial A}{\partial t}+\frac{\partial A}{\partial z}\right)+\delta B & =\frac{\partial W}{\partial \bar{A}}(A, B, \bar{A}, \bar{B}), \\
\mathrm{i}\left(\frac{\partial B}{\partial t}-\frac{\partial B}{\partial z}\right)+\delta A & =\frac{\partial W}{\partial \bar{B}}(A, B, \bar{A}, \bar{B}) .
\end{aligned}
$$

Here, $(A, B) \in \mathbb{C}^{2}, z \in[0, L]$ and $t \geq 0$. The potential function $W$ represents the cubic (Kerr) and higher-order nonlinear terms, i.e. $W=\mathrm{O}(4)$ in a Taylor series at $A=B=0$. We assume that $W$ is invariant under the gauge symmetry of $(1.1):(A, B) \mapsto \mathrm{e}^{\mathrm{i} \varphi}(A, B)$. Spatial reflection in (1.1) exhibits the symmetry $A \rightarrow B, B \rightarrow A, z \rightarrow-z$ and time inversion yields the symmetry $A \rightarrow \bar{B}, B \rightarrow \bar{A}, t \rightarrow-t$. The parameter $\delta \geq 0$ measures the standard deviation of the linear refractive index, that is, $\delta^{2}=\overline{n_{0}^{2}(z)}-\left(\overline{n_{0}(z)}\right)^{2}$, where $n_{0}=n(z, 0)$ and the bar denotes the average of a function on a period of the grating, here.

One specific form of the potential function is

$$
W=-\frac{n}{2}\left(|A|^{4}+4|A|^{2}|B|^{2}+|B|^{4}\right)-m\left(|A|^{2}+|B|^{2}\right)(B \bar{A}+\bar{B} A) .
$$

It occurs when $n\left(z,|E|^{2}\right)=n_{0}(z)+n_{2}(z)|E|^{2}[15]$. The constants $n$ and $m$ measure the average nonlinear index $n=\overline{n_{2}(z)}$ and the standard deviation of the nonlinear index $m^{2}=$ $\overline{n_{2}^{2}(z)}-\left(\overline{n_{2}(z)}\right)^{2}$.

When considered on the entire real line, or when equipped with periodic boundary conditions, the system (1.2) can be viewed as an infinite-dimensional Hamiltonian dynamical system. However, we consider the system (1.2) on finite and semi-infinite intervals $z \in[0, L], L \leq \infty$, with separated boundary conditions. The system then behaves like a dissipative dynamical 
system. In particular, we are interested in solutions of (1.2) with the following boundary conditions:

$$
A(0, t)=I_{\text {in }}^{1 / 2} \mathrm{e}^{\mathrm{i} \theta_{\text {in }}}, \quad B(L, t)=0 .
$$

The specific boundary conditions (1.4) correspond to a physical situation of a uni-directional optical device when the forward (right-travelling) wave $A(z, t)$ is injected at $z=0$, and the backward (left-travelling) wave $B(z, t)$ is inhibited at the right boundary $z=L$, such that $I_{\text {in }}$ and $\theta_{\text {in }}$ are intensity and phase of the incident right-travelling wave at the left boundary.

In case of infinite $L$, we only impose one boundary condition

$$
A(0, t)=I_{\text {in }}^{1 / 2} \mathrm{e}^{\mathrm{i} \theta_{\text {in }}}
$$

and incorporate decay as $z \rightarrow \infty$ in the function space.

The specific boundary conditions (1.4) and (1.5) correspond to transparent boundary conditions for the wave equation, with an inhomogeneous Dirichlet term at the left boundary $z=0$. Most of our methods can be adapted to the case of weak reflection at the boundary, although some of the computations will be much more complicated.

The main question addressed in this paper is: what are the equilibrium structures of (1.2) equipped with the boundary conditions (1.4) or (1.5), and what are their stability properties, for finite, for large, and for infinite length $L$.

Our main results can be summarized as follows. Consider $L<\infty$, first. We show that the coupled-mode system generates a smooth nonlinear semiflow. Spectral stability of stationary light transmission implies asymptotic stability of the equilibrium state for the nonlinear system. Bifurcations can be described on smooth, finite-dimensional center-manifolds. We therefore focus on spectral stability properties of equilibria. Denote by $I_{\text {out }}$ the output intensity, $I_{\text {out }}=|A(L, t)|^{2}$. We first show that for $I_{\text {in }}$ and $I_{\text {out }}$ small, there is a unique, small-amplitude, stationary solution, which is asymptotically stable. Under suitable growth assumptions on the potential $W$, for each value of the output intensity $I_{\text {out }} \geq 0$ there exists a unique value $I_{\text {in }}=\mathcal{I}_{L}\left(I_{\text {out }}\right)$ for a stationary solution of the problem (1.2) with (1.4). Whereas for small amplitudes we have $\mathcal{I}_{L}^{\prime}\left(I_{\text {out }}\right)>0$, there may exist values of $I_{\text {out }}$ with negative-slope transmission function $\mathcal{I}_{L}^{\prime}\left(I_{\text {out }}\right)<0$. We show that stationary solutions with negative slopes of $I_{\text {in }}=\mathcal{I}_{L}\left(I_{\text {out }}\right)$ are always unstable.

In the semi-infinite domain, we consider stationary solutions that decay at infinity, $|A(z)|^{2}=$ $|B(z)|^{2} \sim Q_{\infty} \exp (-\delta z)$. Again, for each value of $Q_{\infty}$ there exists a unique input intensity $I_{\text {in }}=\mathcal{I}_{\infty}\left(Q_{\infty}\right)$ such that there exists a corresponding stationary solution of the problem (1.2) with (1.5). Solutions with small $I_{\text {in }}$ and $Q_{\infty}$ are spectrally stable. Let $Q(z)=|A(z)|^{2}=|B(z)|^{2}$ be the amplitude of a stationary solution of (1.2) on $z \in \mathbb{R}_{+}$. We show that solutions with 
negative-slope transmission characteristic $\mathcal{I}_{\infty}^{\prime}<0$ (or, equivalently, $Q^{\prime}(0)>0$ ) are always unstable.

In the limit when the size of the structure tends to infinity, we show that the renormalized transmission function $\mathcal{I}_{L}\left(\mathrm{e}^{2 \delta L} Q_{\infty} / 2\right)$ converges to the transmission function in the infinite domain, i.e. $\lim _{L \rightarrow \infty} \mathcal{I}_{L}\left(\mathrm{e}^{2 \delta L} Q_{\infty} / 2\right)=\mathcal{I}_{\infty}\left(Q_{\infty}\right)$. The spectrum of the linearized operator in a finite structure converges to that of the linearized operator in a semi-infinite structure as the size of the structure tends to infinity. We give first order expansions for the part of the spectrum approximating the essential spectrum and for the fold point. In particular, we show that in the low-intensity limit, $I_{\text {in }} \ll 1$, the spectrum is confined to $\operatorname{Re} \lambda \leq \delta<0$, uniformly in the size of the domain $L$ and the incident intensity $I_{\text {in }}$. We then give criteria when this spectral stability, uniform with respect to the size of the domain, holds for finite-size intensities.

The paper is concluded with applications of our results to the specific potential in (1.3) arising from the Maxwell equations with Kerr nonlinearities. We show that optical bistability does not occur with the potential $(1.3)$ with $n=0$, i.e. $I_{\text {in }}=\mathcal{I}_{L}\left(I_{\text {out }}\right)$ is one-to-one and the spectrum of the linearized operator is in the left half-plane of $\lambda$. For the potential (1.3) with $m=0$, we analyze numerically real and complex unstable eigenvalues of the linearized operator for optically bistable stationary solutions with negative and positive slopes of the transmission function $I_{\text {in }}=\mathcal{I}_{L}\left(I_{\text {out }}\right)$. We show that the spectrum of the linearized operator for stationary solutions on a negative-slope branch of $\mathcal{I}_{L}\left(I_{\text {out }}\right)$ possesses exactly one real positive eigenvalue. We also show that the stationary solution at the lowest positive-slope branch of $\mathcal{I}_{L}\left(I_{\text {out }}\right)$ is spectrally stable, while the solution at the upper positive slope branches of $\mathcal{I}_{L}\left(I_{\text {out }}\right)$ has a single pair of complex eigenvalues with positive real part.

Technically, we rely on regularity estimates in the spirit of delay differential equations for analysis of bifurcations and asymptotic stability results in finite domains. Instability can be shown based on Evans function methods in the bounded interval or on Leray-Schauder degree arguments. Perturbation methods in the spirit of $[16,17]$, are then employed to derive expansions for spectra in the limit of infinite size. Key points are expansions for the location of the translational zero eigenvalue of the soliton in a large but finite domain, and the location of eigenvalues stemming from the essential spectrum, corresponding to radiation modes, in the infinite domain. Deriving asymptotics for the location of absolute (limiting) spectra under the influence of radiation loss through the boundary is similar to the derivation of expansions in [17]. Numerical computations of unstable eigenvalues are based on winding number arguments for the Evans function.

This paper is organized as follows. We start with the general mathematical framework in Section 2, introducing Hamiltonian formalism and settling regularity issues. We then introduce 
spatial symplectic dynamics and discuss existence and bifurcation diagrams for stationary solutions in both finite and semi-infinite structures in Section 3. We review recent literature on optical bistability in Section 4. In Section 5, we formulate and prove our main results on stability and instability in finite domains, based on degree arguments, and on center-manifold theory. In Section 6, we give an alternative proof based on Evans function arguments. We then extend the latter proof to semi-infinite structures in Section 7. In Section 8, we derive expansions for the location of the zero eigenvalue of the soliton and the absolute spectrum when truncating the semi-infinite domain $z \in[0, \infty)$ to a large interval $z \in[0, L]$. Explicit analytical and numerical computations for the potential (1.3) are described in Section 9. Section 10 summarizes the main results of the paper. Appendix A contains formulas for the kernel of the adjoint linearized operator as well as some useful relations for the stationary solutions of the system. Appendix B contains formulas for the derivative of the Evans function.

Acknowledgments D.P. was partially supported by the NSERC grant RGP-238931-01. A.S. was partially supported by the NSF grant DMS-0203301.

\section{Hamiltonian formalism and local existence}

Consider the phase space $X=\left(L^{2}(D, \mathbb{C})\right)^{4}$ on an interval $D \subset \mathbb{R}$ with standard scalar product $(\cdot, \cdot)_{X}$ and symplectic structure $\Omega(\cdot, \cdot)$ for $\mathbf{A}=\left(A_{1}, B_{1}, A_{2}, B_{2}\right) \in X$

$$
\begin{aligned}
\left(\mathbf{A}_{I}, \mathbf{A}_{I I}\right)_{X} & =\int_{D} \sum_{j=1}^{2}\left(A_{I, j} \bar{A}_{I I, j}+B_{I, j} \bar{B}_{I I, j}\right) d z \\
\Omega\left(\mathbf{A}_{I}, \mathbf{A}_{I I}\right) & =\left(\mathbf{A}_{I}, \mathcal{J} \mathbf{A}_{I I}\right)_{X} .
\end{aligned}
$$

Here, the symplectic matrix $\mathcal{J}=\operatorname{diag}(\mathrm{i}, \mathrm{i},-\mathrm{i},-\mathrm{i})$ is skew-symmetric and unitary. Most of the time, we restrict to the subspace $Y \subset X$ where $A_{2}=\bar{A}_{1}$, and $B_{2}=\bar{B}_{1}$. Consider the Hamiltonian function

$$
\mathcal{H}[A, \bar{A}, B, \bar{B}]=\int\left[\frac{\mathrm{i}}{2}\left(\bar{A} A_{z}-A \bar{A}_{z}\right)-\frac{\mathrm{i}}{2}\left(\bar{B}_{z}-B \bar{B}_{z}\right)+\delta(A \bar{B}+\bar{A} B)-W(A, B, \bar{A}, \bar{B})\right] d z,
$$

which is defined on a dense subset of $Y$. If we consider $D=\mathbb{R}$ or $D=[0, L]$ with periodic boundary conditions, we find the coupled mode system (1.2) as the Hamiltonian system on $Y$

$$
A_{t}=\mathrm{i} \nabla_{\bar{A}} \mathcal{H}, \quad B_{t}=\mathrm{i} \nabla_{\bar{B}} \mathcal{H}
$$

where the derivatives on the right side are understood as the components of the gradient of the densely defined functional. As a consequence, the Hamiltonian $\mathcal{H}$ is preserved as 
a function of time for sufficiently smooth solutions. The gauge symmetry in the potential $(A, B) \mapsto \mathrm{e}^{\mathrm{i} \varphi}(A, B)$ is generated by

$$
\mathcal{G}_{\varphi}[A, \bar{A}, B, \bar{B}]=\frac{1}{2} \int_{D}(A \bar{A}+B \bar{B}) d z .
$$

By Noether's theorem, the $L^{2}$ norm on $Y$ of solutions is therefore an additional conserved quantity. Similarly, the translation invariance in $z$ generates yet another conserved quantity

$$
\mathcal{G}_{z}[A, \bar{A}, B, \bar{B}]=\frac{\mathrm{i}}{2} \int_{D}\left(\bar{A} A_{z}-A \bar{A}_{z}+\bar{B} B_{z}-B \bar{B}_{z}\right) d z .
$$

None of these functionals is preserved when we consider the system on a finite interval with boundary conditions (1.4), for $L<\infty$ or (1.5), for $L=\infty$. We note that there exist other (symmetric) boundary conditions that preserve the Hamiltonian structure, e.g. $A(z)=B(z)$ for $z=0, L$.

We start with an analysis of the problem (1.2) and (1.4) with $I_{\text {in }}=0$, linearized in the trivial state $A=B=0$. We consider first the case $L<\infty$ and define the linear operator

$$
\begin{aligned}
\mathcal{L}: \mathcal{D}(\mathcal{L}) \subset Y & \rightarrow Y \\
(A, B, \bar{A}, \bar{B}) & \mapsto \quad\left(\mathrm{i} A_{z}+\delta B,-\mathrm{i} B_{z}+\delta A,-\mathrm{i} \bar{A}_{z}+\delta \bar{B}, \mathrm{i} \bar{B}_{z}+\delta \bar{A}\right),
\end{aligned}
$$

with domain of definition

$$
\mathcal{D}(\mathcal{L})=\left(H^{1}(0, L)\right)^{4} \cap\{A(0)=\bar{A}(0)=B(L)=\bar{B}(L)=0\}
$$

The composition $\mathcal{J} \mathcal{L}$ possesses the same domain of definition and represents the linear terms in the coupled-mode system (1.2).

Lemma 2.1 The operator $\mathcal{J} \mathcal{L}$ generates a strongly continuous contraction semigroup on either $X$ or $Y$, denoted by $\exp (\mathcal{J} \mathcal{L} t)$.

Proof. We claim that $(\mathcal{J} \mathcal{L} \mathbf{A}, \mathbf{A})_{Y} \leq 0$ for all $\mathbf{A}=(A, B, \bar{A}, \bar{B})$ in $\mathcal{D}(\mathcal{L})$. Indeed, a short computation shows that

$$
(\mathcal{J} \mathcal{L} \mathbf{A}, \mathbf{A})_{Y}=\int_{0}^{L} \frac{\mathrm{d}}{\mathrm{d} z}\left(|B|^{2}-|A|^{2}\right) d z=-|A(L)|^{2}-|B(0)|^{2} \leq 0 .
$$

Together with a standard resolvent estimate, this shows that $\mathcal{J} \mathcal{L}$ generates a contraction semigroup on $Y$, invoking the Lumer-Philips Theorem; see for example [12, Thm 4.3]. The operator $\mathcal{J} \mathcal{L}$ considered on the entire space $X$ possesses block diagonal structure. In each block, $\mathcal{J} \mathcal{L}$ is isomorphic to its restriction on $Y$, which proves the lemma.

The following proposition gives a more detailed information about the spectrum of the linear operator $\mathcal{J} \mathcal{L}$. 
Proposition 2.2 Let the linear operator $\mathcal{J} \mathcal{L}$ be defined by (2.6) with domain (2.7) for $0<$ $L<\infty$. Then the spectrum of $\mathcal{J} \mathcal{L}$ consists of isolated eigenvalues of finite multiplicities and is implicitly given through

$$
\Gamma:=\operatorname{spec}(\mathcal{J} \mathcal{L})=\{\lambda=-\nu \operatorname{coth}(\nu L) ; \nu \in \mathbb{C}, \operatorname{Re} \nu \geq 0: D(\nu)=0\}
$$

where

$$
D(\nu)=\frac{\sinh ^{2}(\nu L)}{(\nu L)^{2}}+\frac{1}{(\delta L)^{2}}
$$

The spectrum of the semigroup is discrete and coincides with $\overline{\exp (\Gamma)}$. For some constants $C, \eta>0$, we have

$$
|\exp (\mathcal{J} \mathcal{L} t)|_{X \rightarrow X} \leq C \mathrm{e}^{-\eta t}
$$

Proof. As a bounded perturbation of the first derivative operators $\operatorname{diag}\left(-\partial_{z}, \partial_{z},-\partial_{z}, \partial_{z}\right)$, $\mathcal{J} \mathcal{L}-\lambda$ possesses compact resolvent for sufficiently large positive Re $\lambda$. Therefore, the spectrum consists of isolated eigenvalues of finite multiplicities, only. Nontrivial solutions of the eigenvalue problem satisfy the differential equation

$$
-A_{z}+\mathrm{i} \delta B=\lambda A, \quad B_{z}+\mathrm{i} \delta A=\lambda B
$$

with boundary conditions $A(0)=B(L)=0$. Substituting the Ansatz $A=A_{0} \exp (\nu z)$, $B=B_{0} \exp (\nu z)$, we find $\mathrm{i} \delta B_{0}=(\nu+\lambda) A_{0}$ and $\nu^{2}=\delta^{2}+\lambda^{2}$. For $\nu \neq 0$, the general solution to $(2.12)$ is

$$
\left[\begin{array}{l}
A \\
B
\end{array}\right]=c_{1} \boldsymbol{\psi}_{\nu}(z)+c_{2} \boldsymbol{\psi}_{-\nu}(z), \quad \boldsymbol{\psi}_{\nu}(z)=\frac{1}{\delta}\left(\begin{array}{c}
\delta \\
-\mathrm{i}(\lambda+\nu)
\end{array}\right) \mathrm{e}^{\nu z}
$$

where $\nu=\sqrt{\delta^{2}+\lambda^{2}}$ such that $\operatorname{Re}(\nu) \geq 0$. The boundary condition at $z=0$ implies $c_{2}=-c_{1}$. At $z=L$, we find

$$
(\lambda+\nu) \mathrm{e}^{\nu L}=(\lambda-\nu) \mathrm{e}^{-\nu L}
$$

As a result, $\lambda=-\nu \operatorname{coth}(\nu L)$. Substituting $\lambda$ in the equation $\nu^{2}=\delta^{2}+\lambda^{2}$, we find the condition $D(\nu)=0$, where $D(\nu)$ is given by (2.10). This proves the claims on the spectrum of $\mathcal{L}$. Since the essential spectrum of $\mathcal{L}$ is empty, spectral mapping holds [1, p 95]. From $D(\nu)=0$, we see that there are no purely imaginary and purely real eigenvalues of $\mathcal{L}$. Also, all eigenvalues $\lambda$ and $\nu$ occur in complex conjugate pairs. In the limit $|\operatorname{Im} \lambda| \rightarrow \infty$, we can expand (2.14) and find

$$
\lambda \sim-\frac{1}{L} \log \left(\frac{2 \pi \ell}{\delta L}\right)+\frac{\mathrm{i} \pi \ell}{L}
$$

where $\ell$ is non-zero integer. Splitting real and imaginary part, we find

$$
\operatorname{Re} \lambda \sim-\frac{1}{L} \log \left(\frac{2|\operatorname{Im} \lambda|}{\delta}\right) .
$$


Invoking the energy estimate (2.8), this shows that the spectral radius of the semigroup is strictly less than one, and implies the contraction estimate (2.11).

We computed the complex eigenvalues of the operator $\mathcal{L}$ numerically for $\delta=0.1$ and $L=$ 10; see Fig. 1(a). All complex eigenvalues are in the left half-plane of $\lambda$. The asymptotic approximation (2.16) is shown on Fig. 1(a) by the dotted curve. The approximation is very good even for small values of $\lambda$. The eigenvalues closest to the origin $\lambda=0$ have the largest real part: $\operatorname{Re}(\lambda) \approx-0.1755$ and $\operatorname{Im}(\lambda) \approx \pm 0.2666$ for $\delta=0.1$ and $L=10$.

Considered on $X$, all eigenvalues of $\mathcal{J} \mathcal{L}$ are double. If $\delta=0$, the spectrum is empty for all $L<\infty$. As the size $L$ increases, dissipation in (2.16) becomes weaker. The spectrum for large $L$ is shown on Fig. 1(b) for $\delta=0.1$ and $L=100$.

We turn to the case $L=\infty$ and define the operator $\mathcal{L}_{\infty}$ by $(2.6)$ on

$$
\mathcal{D}\left(\mathcal{L}_{\infty}\right)=\left(H^{1}(0, \infty)\right)^{4} \cap\{A(0)=\bar{A}(0)=0\}
$$

as a closed operator in $Y$. Again, $\mathcal{J} \mathcal{L}_{\infty}$ is a closed operator with the same domain of definition.

Lemma 2.3 The spectrum of the closed operator $\mathcal{J} \mathcal{L}_{\infty}$ consists of continuous spectrum

$$
\Gamma_{\infty}:=\operatorname{spec}\left(\mathcal{J} \mathcal{L}_{\infty}\right)=\{\lambda: \operatorname{Re}(\lambda)=0, \operatorname{Im}(\lambda)=(-\infty,-\delta] \cup[\delta, \infty)\}
$$

Proof. The essential spectrum can be computed from the operator $\mathcal{L}_{\infty}$ on the entire real line by Fourier transform. It consists of the set $\lambda^{2}=-\delta^{2}+\nu^{2}$, where $\nu=\mathrm{i} k$ and $k \in \mathbb{R}$. The eigenvalue problem (2.12) does not possess nontrivial bounded solutions satisfying $A(0)=0$ for $\lambda \in \mathbb{C} / \Gamma_{\infty}$, i.e. in the complement of the essential spectrum. Indeed, the solution (2.13) with $c_{2}=-c_{1}$ has both decaying and growing terms as $z \rightarrow \infty$ for $\operatorname{Re}(\nu)>0$.

In the case $L=\infty$, the semigroup does not seem to regularize solutions. This is obvious in the case of pure transport $\delta=0$. On the contrary, for bounded domains $L<\infty$, we show that the semigroup still has some smoothing properties, which are important for solving the nonlinear problem.

We define $H_{\mathrm{bc}}^{1}:=\mathcal{D}(\mathcal{L})$, the domain of definition, equipped with the graph norm $|u|_{H_{\mathrm{bc}}^{1}}=$ $|u|_{X}+|\mathcal{L} u|_{X}$, which is equivalent to the norm in $H^{1}$.

Lemma 2.4 There are $C, \eta>0$ such that for all $t>0$, and any $\mathbf{r} \in C^{0}\left(\left(H^{1}\right)^{4},[0, T)\right)$, the solution A of

$$
\mathbf{A}_{t}=\mathcal{J} \mathcal{L} \mathbf{A}+\mathbf{r}(t), \quad \mathbf{A}(0) \in H_{\mathrm{bc}}^{1}
$$



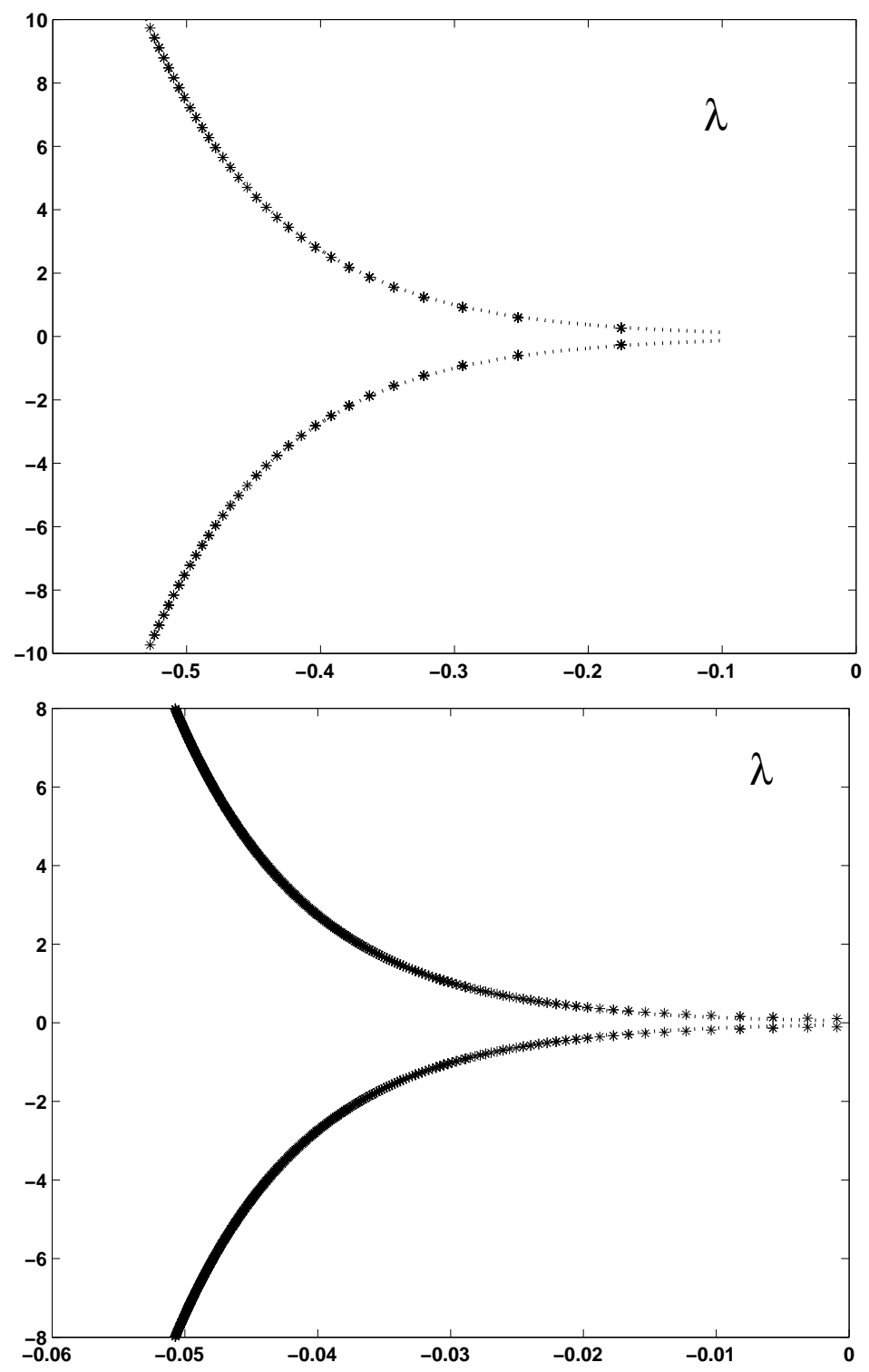

Figure 1: The stable spectrum of operator $\mathcal{J} \mathcal{L}$ for $\delta=0.1, L=10$ (a) and for $\delta=0.1, L=100$ (b). 
belongs to $C^{0}\left(H_{\mathrm{bc}}^{1},[0, T)\right)$ with bound

$$
\sup _{0 \leq t \leq T}|\mathbf{A}(t)|_{H_{\mathrm{bc}}^{1}} \leq C(T) \sup _{t}|\mathbf{r}|_{H^{1}}
$$

Moreover, the time- $T$ map $\exp (\mathcal{J} \mathcal{L} T)$ for $T>L$ is a compact operator on $X, H_{\mathrm{bc}}^{1}$, or $\left(H^{1}\right)^{4}$.

Proof. The lemma is obvious in the case of pure transport $\delta=0$, where we can use characteristics. We can extend the initial conditions $A_{0}(z), B_{0}(z)$, and the right-hand-side functions $r_{A}(z), r_{B}(z)$ to $z \in \mathbb{R}$, setting $r_{A}(z)=r_{B}(z)=A_{0}(z)=B_{0}(z)=0$ for $z \notin[0, L]$. The solution for $\delta=0$ is then defined through

$$
\begin{aligned}
& A(z, t)=A_{0}(z-t)+\int_{0}^{t} r_{A}(z-t+\tau, \tau) \mathrm{d} \tau \\
& B(z, t)=B_{0}(z+t)+\int_{0}^{t} r_{B}(z+t-\tau, \tau) \mathrm{d} \tau .
\end{aligned}
$$

In case $\delta \neq 0$, the same formula gives

$$
\begin{aligned}
& A(z, t)=A_{0}(z-t)+\int_{0}^{t}\left[r_{A}(z-t+\tau, \tau)+\mathrm{i} \delta B(z-t+\tau, \tau)\right] \mathrm{d} \tau \\
& B(z, t)=B_{0}(z+t)+\int_{0}^{t}\left[r_{B}(z+t-\tau, \tau)+\mathrm{i} \delta A(z+t-\tau, \tau)\right] \mathrm{d} \tau .
\end{aligned}
$$

The bounds (2.20) can be obtained from this representation and a fixed point argument for small $t$, observing that, indeed, $A(0, t)=B(L, t)=0$.

It remains to show compactness. Again, we exploit (2.22), with $\mathbf{r}(t) \equiv 0$. For $A_{0}, B_{0}$ in $X$, the terms $A_{0}(z-t)$ and $B_{0}(z+t)$ vanish for $t>L$. The integral terms belong to $H_{\mathrm{bc}}^{1}$, since they satisfy the boundary conditions, and, for example,

$$
\partial_{z} A=\mathrm{i} \delta \int_{0}^{t} \partial_{z} B(z-t+\tau, \tau) \mathrm{d} \tau, \quad t>L .
$$

If we now substitute the expression for $B$ from (2.22) and change the order of integration, we see after a short manipulation that $\partial_{z} A \in H^{1}$ if $B \in H^{1}$. A similar argument for $B$ then proves the lemma.

Since $H^{1}$ is an algebra, the nonlinearities are as smooth as the second derivative of the potential $W$, as mappings from $H^{1} \times H^{1}$ into itself. A standard variation of constant formula together with the contraction mapping principle gives the following existence result for the nonlinear equation with inhomogeneous boundary condition (1.4). Denote by $H_{\text {af }}^{1}$ the affine subspace of functions in $\left(H^{1}\right)^{4}$ which satisfy (1.4).

Proposition 2.5 The coupled mode equation (1.2) generates a strongly continuous, local semiflow $\Phi_{t}(\mathbf{A})$ on $H_{\mathrm{af}}^{1}$. The semiflow $\Phi_{t}(\cdot)$ is smooth if the potential $W$ is smooth, with derivatives uniformly bounded on bounded subsets of $H_{\mathrm{af}}^{1}$. 
The results on spectral mapping in Proposition 2.2 and the local existence result in Proposition 2.5 immediately imply existence of locally invariant stable, unstable, center manifolds near an equilibrium point. In particular, spectral stability (spectrum contained in $\operatorname{Re} \lambda \leq-\eta^{\prime}<0$ ) implies local asymptotic nonlinear stability.

More specifically, let $\mathbf{A}_{*} \in H_{\mathrm{af}}^{1}$ be a stationary solution $\Phi_{t}\left(\mathbf{A}_{*}\right)=0$. Decompose the solution into the stationary part and the perturbation

$$
\mathbf{A}(z, t)=\mathbf{A}_{*}(z)+\mathbf{A}_{\mathrm{p}}(z, t) .
$$

Then the perturbation $\mathbf{A}_{\mathrm{p}}(z, t)$ solves

$$
\frac{\mathrm{d}}{\mathrm{d} t} \mathbf{A}_{p}=\mathcal{J} \mathcal{L}_{*} \mathbf{A}_{\mathrm{p}}+\mathrm{O}\left(\left|\mathbf{A}_{\mathrm{p}}\right|_{H_{\mathrm{af}}^{1}}^{2}\right)
$$

Here the linearization $\mathcal{J} \mathcal{L}_{*}$ about the stationary solution $\mathbf{A}_{*}$ is defined by

$$
\mathcal{L}_{*}=\mathcal{L}-\mathcal{W}
$$

where

$$
\mathcal{W}=\left[\begin{array}{ll}
W_{1} & W_{2} \\
\bar{W}_{2} & \bar{W}_{1}
\end{array}\right]
$$

and

$$
W_{1}=\left[\begin{array}{cc}
\partial_{\bar{A} A} W & \partial_{\bar{A} B} W \\
\partial_{\bar{B} A} W & \partial_{\bar{B} B} W
\end{array}\right], \quad W_{2}=\left[\begin{array}{cc}
\partial_{\bar{A} \bar{A}} W & \partial_{\bar{A} \bar{B}} W \\
\partial_{\bar{B} \bar{A}} W & \partial_{\bar{B} \bar{B}} W
\end{array}\right]
$$

Since $\mathbf{A}(z, t)$ and $\mathbf{A}_{*}(z)$ satisfy the inhomogeneous boundary conditions in $z=0$, the boundary conditions for the difference $\mathbf{A}_{\mathrm{p}}=\left[A_{\mathrm{p}}, B_{\mathrm{p}}, \bar{A}_{\mathrm{p}}, \bar{B}_{\mathrm{p}}\right]$ are homogeneous

$$
A_{\mathrm{p}}(0, t)=\bar{A}_{\mathrm{p}}(0, t)=0, \quad B_{\mathrm{p}}(L, t)=\bar{B}_{\mathrm{p}}(L, t)=0 .
$$

In other words, the perturbation term $\mathbf{A}_{\mathrm{p}}(z, t)$ is not allowed to modify the intensity of the incident wave $I_{\text {in }}$. Stability or instability of stationary solution $\mathbf{A}_{*}(z)$ is considered with respect to internal perturbations of the light waves inside the periodic structure, alone.

The linear operator $\mathcal{J} \mathcal{L}_{*}$ on $H_{\mathrm{bc}}^{1}$ is a bounded perturbation of $\mathcal{J} \mathcal{L}$ and shares most regularity properties with $\mathcal{J} \mathcal{L}$.

Corollary 2.6 The operator $\mathcal{J} \mathcal{L}_{*}$ with domain of definition $H_{\mathrm{bc}}^{1}$ generates a strongly continuous semigroup $\Phi_{t}^{\prime}$ on $Y$. Moreover, $\left(\mathcal{J} \mathcal{L}_{*}-\lambda\right)^{-1}$ is a compact operator, wherever it exists. The boundary smoothing of Lemma 2.4 holds with $\mathcal{J} \mathcal{L}$ replaced by $\mathcal{J} \mathcal{L}_{*}$. Again, $\Phi_{T}^{\prime}$ is compact for $T>L$.

Proof. The proof is the same as for the linear operator $\mathcal{J} \mathcal{L}$ in Lemma 2.4. 
Assume that there exists an invariant decomposition of $Y$ into closed subspaces $Y=\mathcal{E}^{\mathrm{s}} \oplus \mathcal{E}^{\mathrm{c}} \oplus$ $\mathcal{E}^{\mathrm{u}}$. Any of the subspaces is allowed to be trivial. Note however that, by compactness, $\mathcal{E}^{\mathrm{c}} \oplus \mathcal{E}^{\mathrm{u}}$ is finite-dimensional. Assume that there exist constants $C, \eta>0$ such that for any $\varepsilon>0$, we have

$$
\begin{aligned}
& \left|\Phi_{t}^{\prime}\right|_{\mathcal{E}^{\mathrm{s}} \mapsto \mathcal{E}^{\mathrm{s}}} \leq C \mathrm{e}^{-\eta t}, \text { for all } t \geq 0 \\
& \left|\Phi_{t}^{\prime}\right|_{\mathcal{E}^{\mathrm{c}} \mathcal{E}^{\mathrm{c}}} \leq C \mathrm{e}^{\varepsilon|t|}, \text { for all } t \in \mathbb{R} \\
& \left|\Phi_{t}^{\prime}\right|_{\mathcal{E}^{\mathrm{u}_{\vdash}}} \leq \mathcal{E}^{\mathrm{u}} \leq C \mathrm{e}^{-\eta|t|}, \text { for all } t \leq 0 \text {. }
\end{aligned}
$$

Here $\Phi_{t}^{\prime}:=\left(\Phi_{-t}^{\prime}\right)^{-1}$ if $t<0$.

Proposition 2.7 Under the above assumptions, there exist stable, center, and unstable manifolds $\mathcal{W}^{j}, j=\mathrm{s}, \mathrm{c}, \mathrm{u}$, containing the equilibrium $\mathbf{A}_{*}(z)$. The manifolds are locally invariant under $\Phi_{t}, C^{1}$, and the tangent space in $\mathbf{A}_{*}(z)$ is given by $\mathcal{E}^{j}, j=\mathrm{s}, \mathrm{c}, \mathrm{u}$. The center-manifold $\mathcal{W}^{\mathrm{c}}$ contains all solutions, which stay in a sufficiently small neighborhood of the equilibrium $\mathbf{A}_{*}(z)$. If $\mathcal{E}^{\mathrm{u}}=\{0\}$, then $\mathcal{W}^{\mathrm{c}}$ attracts all solutions for $t \rightarrow \infty$, which remain in a sufficiently small neighborhood of $\mathbf{A}_{*}(z)$. If $\mathcal{E}^{\mathrm{u}}=\mathcal{E}^{\mathrm{c}}=\{0\}$, then $\mathbf{A}_{*}(z)$ is asymptotically stable in $Y$.

Proof. Compactness of the semiflow ensures existence of spectral projections. Cut-off functions as needed for the construction of center manifolds are provided by the norm in the Hilbert space $H_{\mathrm{bc}}^{1}$. After a cut-off for the nonlinearity $W^{\prime}$, acting on $\left(H^{1}\right)^{4}$, the manifolds are constructed as invariant manifolds for the time- $T$ map of the nonlinear semiflow. For a reference on the construction of invariant manifolds for maps in metric spaces; see [18].

Remark 2.8 The manifolds $W^{\mathrm{s}, \mathrm{c}, \mathrm{u}}$ are $C^{k}$ for any fixed $k$, if the potential $W$ is sufficiently smooth in the system (1.2). Also, dependence of the system (1.2) on parameters, such as the input intensity $I_{\mathrm{in}}$, is smooth.

Remark 2.9 The results of Lemma 2.4, Proposition 2.5, Corollary 2.6, and Proposition 2.7 carry over to the case $L=\infty$, except for the claims on compactness of resolvents and timeT-maps of semigroups.

Summarizing the results in this section, we have shown that stability properties on finite intervals are typically determined by spectral properties and instabilities are induced by point spectrum crossing the imaginary axis. This is in sharp contrast to the coupled mode system (1.2) when considered on the entire real line or even in the semi-infinite domain $[0, \infty)$. We have also shown that the zero solution $\mathbf{A}_{*}(z) \equiv 0$ for $I_{\text {in }}=0$ is asymptotically stable for the full nonlinear equation. The small perturbation terms $\mathbf{A}_{\mathrm{p}}(z, t)$ decay exponentially with $t$. Finite 
length of the structure is essential to this type of asymptotic stability, which can never occur in a Hamiltonian system. Perturbations radiate through the transparent boundary conditions (2.28), which eventually causes exponential decay.

\section{$3 \quad$ Stationary light transmission}

Here we study stationary solutions $\mathbf{A}_{*}(z)$ of the system (1.2) with boundary conditions (1.4) for $L<\infty$ and (1.5) for $L=\infty$. Consider the system of differential equations:

$$
\begin{aligned}
\mathrm{i} \frac{\mathrm{d} a}{\mathrm{~d} z}+\delta b & =\frac{\partial W}{\partial \bar{a}}(a, b, \bar{a}, \bar{b}), \\
-\mathrm{i} \frac{\mathrm{d} b}{\mathrm{~d} z}+\delta a & =\frac{\partial W}{\partial \bar{b}}(a, b, \bar{a}, \bar{b}),
\end{aligned}
$$

and the corresponding complex conjugate equations. Any solution to the system (3.1), which satisfies the boundary condition

$$
a(0)=I_{\text {in }}^{1 / 2} \mathrm{e}^{\mathrm{i} \theta_{\text {in }}}, \quad b(L)=0,
$$

yields a stationary solution $\mathbf{A}_{*}=(a, b, \bar{a}, \bar{b})$ to the coupled-mode system (1.2).

Although the temporal dynamics of the system (1.2) is not Hamiltonian with the given boundary conditions (1.4) and (1.5), the system (3.1) for stationary solutions possesses a Hamiltonian structure. Define the Hamiltonian function $h$ on the phase space $\mathbf{a}=(a, b, \bar{a}, \bar{b}) \in \mathbb{C}^{4}$ through

$$
h(a, \bar{a}, b, \bar{b})=\delta(\bar{a} b+a \bar{b})-W(a, b, \bar{a}, \bar{b}) .
$$

We equip the phase space $\mathbf{a}=\left(a_{1}, b_{1}, a_{2}, b_{2}\right)$ with the standard inner product

$$
\left(\mathbf{a}_{I}, \mathbf{a}_{I I}\right)=\sum_{j=1}^{2}\left(a_{I, j} \bar{a}_{I I, j}+b_{I, j} \bar{b}_{I I, j}\right)
$$

and a non-standard symplectic structure

$$
\omega\left(\mathbf{a}_{I}, \mathbf{a}_{I I}\right)=\left(\mathbf{a}_{I}, j \mathbf{a}_{I I}\right)
$$

with symplectic matrix $j=\operatorname{diag}(\mathrm{i},-\mathrm{i},-\mathrm{i}, \mathrm{i})$. Again, $a_{2}=\bar{a}_{1}$ and $b_{2}=\bar{b}_{1}$ on the subspace $Y \subset X$. The corresponding Hamiltonian system reads

$$
a_{z}=\mathrm{i} \frac{\partial h}{\partial \bar{a}}, \quad b_{z}=-\mathrm{i} \frac{\partial h}{\partial \bar{b}},
$$

together with the corresponding complex conjugate equation. The time inversion of the coupled-mode equations (1.2) provides the symmetry of the stationary system (3.5): $a \rightarrow \bar{b}$, $b \rightarrow \bar{a}$. The spatial reflection induces the reversibility symmetry: $a \rightarrow b, b \rightarrow a, z \rightarrow-z$. 
Besides the Hamiltonian $h$, the phase equivariance $\mathbf{a} \mapsto \mathrm{e}^{\mathcal{J} \varphi} \mathbf{a}$ enforces an additional conserved quantity, namely the pointwise transmission intensity

$$
I_{\mathrm{pt}}=|a|^{2}-|b|^{2}
$$

We define the output (transmitted) intensity and reflected intensity as

$$
I_{\text {out }}=|a(L)|^{2}, \quad I_{\text {ref }}=|b(0)|^{2} .
$$

Conservation of the pointwise intensity $I_{\mathrm{pt}}$ results in the balance equation

$$
I_{\text {in }}=I_{\text {out }}+I_{\text {ref }}
$$

The boundary conditions (3.2) fix the values of the conserved quantities to

$$
h=h_{s}\left(I_{\text {out }}\right), \quad I_{\mathrm{pt}}=I_{\text {out }},
$$

where $h_{s}=-W\left(I_{\text {out }}^{1 / 2}, 0, I_{\text {out }}^{1 / 2}, 0\right)$. As a Hamiltonian system with two degrees of freedom and two conserved quantities $h$ and $I_{\mathrm{pt}}$, the system (3.5) is integrable. Solutions to the boundaryvalue problem (3.1)-(3.2) can be constructed from a shooting argument. Indeed, solve (3.1) backwards in spatial time $z$ with the "initial" value: $a(L)=I_{\text {out }}^{1 / 2} \mathrm{e}^{\mathrm{i} \theta_{\text {out }}}$ and $b(L)=0$. Because of the phase (gauge) invariance, we can always fix $\theta_{\text {out }}=0$. By factoring the balance equation (3.6), the stationary solution is parameterized as

$$
a(z)=\sqrt{I_{\text {out }}+Q(z)} \mathrm{e}^{\mathrm{i} \theta(z)}, \quad b(z)=\sqrt{Q(z)} \mathrm{e}^{\mathrm{i} \phi(z)},
$$

with the "initial" condition $Q(L)=0$ and $\theta(L)=0$. At the left boundary, we have $I_{\text {ref }}=$ $I_{\text {in }}-I_{\text {out }}=Q(0)$.

Lemma 3.1 There exists a maximal output intensity $0 \leq I_{\lim } \leq \infty$, such that for all output intensities below this value, i.e. for $0 \leq I_{\text {out }}<I_{\text {lim }}$, there exists a unique (up to complex phase shift) stationary solution $\mathbf{A}_{*}(z)$ to (3.1) with real input intensity $I_{\mathrm{in}}=\mathcal{I}_{L}\left(I_{\text {out }}\right) \geq 0$, where $\mathcal{I}_{L}(0)=0$, and smoothly depending on the prescribed output intensity $I_{\text {out }}$. If $I_{\mathrm{lim}}<\infty$, then the unique branch of solutions diverges to infinity for $I_{\text {out }} \rightarrow I_{\mathrm{lim}}$.

Proof. By smooth dependence on the initial data, the value of the solutions to the ODE (3.1) in $z=0$ depends smoothly on the initial data in $z=L$ as long as the solution does not blow up at a finite spatial time $z_{0} \in(0, L)$. At $I_{\text {out }}=0$, we find the spatially homogeneous equilibrium $\mathbf{A}_{*}(z) \equiv \mathbf{0}$ such that $I_{\text {in }}=\mathcal{I}_{L}(0)=0$. At $I_{\text {out }}$ small, the solution $\mathbf{A}_{*}(z)$ remains in a neighborhood of the equilibrium $\mathbf{A}_{*}(z) \equiv \mathbf{0}$ for finite spatial time $z$, which excludes blowup. Therefore, there exists $0<I_{\lim } \leq \infty$ such that the solution $\mathbf{A}_{*}(z)$ is unique and finite for any output intensity $0 \leq I_{\text {out }}<I_{\text {lim }}$. 
We emphasize that for a prescribed value of the boundary-value parameter $I_{\text {in }}$, there may exist several output intensities $I_{\text {out }}^{j}$ such that $I_{\text {in }}=\mathcal{I}_{L}\left(I_{\text {out }}^{j}\right)$. In other words, the solution branch, with $I_{\text {out }}$ plotted over the parameter $I_{\text {in }}$ may have fold points where $\mathcal{I}_{L}^{\prime}\left(I_{\text {out }}\right)=0$. The slopes $\mathcal{I}_{L}^{\prime}\left(I_{\text {out }}\right)$ might be negative for some values of $I_{\text {out }}$.

As an example, we consider the system (3.1)-(3.2) with specific potential $W$ defined in (1.3). By exploiting the parameterization (3.10), it is shown in [15] that $Q(z)$ is a positive solution of the nonlinear problem:

$$
\left(\frac{\mathrm{d} Q}{\mathrm{~d} z}\right)^{2}=Q\left(I_{\text {out }}+Q\right)\left[4\left(\delta+m\left(I_{\text {out }}+2 Q\right)\right)^{2}-9 n^{2} Q\left(I_{\text {out }}+Q\right)\right],
$$

with the boundary conditions $Q(0)=I_{\text {in }}-I_{\text {out }}$ and $Q(L)=0$. The input-output transmission function $I_{\text {in }}=\mathcal{I}_{L}\left(I_{\text {out }}\right)$ is shown on Fig. 2(a) for $n=1, \delta=0.25, m=0$ and on Fig. 2(b) for $n=0, \delta=0.1, m=5$ (solid curve) and $n=0, \delta=0.1, m=-5$ (dashed curve). The length of the structure is fixed at $L=10$.

The stationary solutions exist for all output intensities $0 \leq I_{\text {out }}<\infty$ on Fig. 2(a), whereas the output intensities $I_{\text {out }}$ are bounded by the limiting value such that $0 \leq I_{\text {out }}<I_{\text {lim }}<\infty$ on Fig. 2(b). The case on Fig. 2(a) is generally described as optical bistability, whereas the case on Fig. 2(b) is all-optical limiting. An elementary analysis of (3.11) for large $Q$ shows that optical bistability occurs for $\left(16 m^{2}-9 n^{2}\right)<0$ and all-optical limiting occurs for $\left(16 m^{2}-9 n^{2}\right)>0$.

We now turn to the semi-infinite structures, when $L=\infty$. We are interested in localized solutions, that is, we impose the boundary conditions

$$
a(0)=I_{\text {in }}^{1 / 2} \mathrm{e}^{\mathrm{i} \theta_{\text {in }}}, \quad \lim _{z \rightarrow \infty} a(z)=\lim _{z \rightarrow \infty} b(z)=0 .
$$

Existence of non-decaying solutions can, in general, not be excluded. For example, the differential equation (3.1) could possess nontrivial equilibria $\mathbf{A}_{*}(z) \neq 0$ for $L<\infty$ and $I_{\text {out }} \neq 0$, which would then generate bounded solutions on $z \in[0, \infty)$ for suitable input intensities $I_{\text {in }}$. In order to understand the asymptotic behavior of possible localized stationary solutions when $z \rightarrow \infty$, we study the linearization of (3.1) at $a=b=0$. Since the potential is purely nonlinear, we find

$$
a_{z}=\mathrm{i} \delta b, \quad b_{z}=-\mathrm{i} \delta a .
$$

Two solutions exist in the form:

$$
\left[\begin{array}{l}
a_{ \pm} \\
b_{ \pm}
\end{array}\right]=\mathrm{e}^{ \pm \delta z}\left[\begin{array}{c}
1 \\
\mp \mathrm{i}
\end{array}\right]
$$

With the normalization $\delta>0$, the solution $\left(a_{+}, b_{+}\right)$diverges as $z \rightarrow+\infty$ and the solution $\left(a_{-}, b_{-}\right)$converges as $z \rightarrow+\infty$. They span the linear unstable and stable subspace of the 

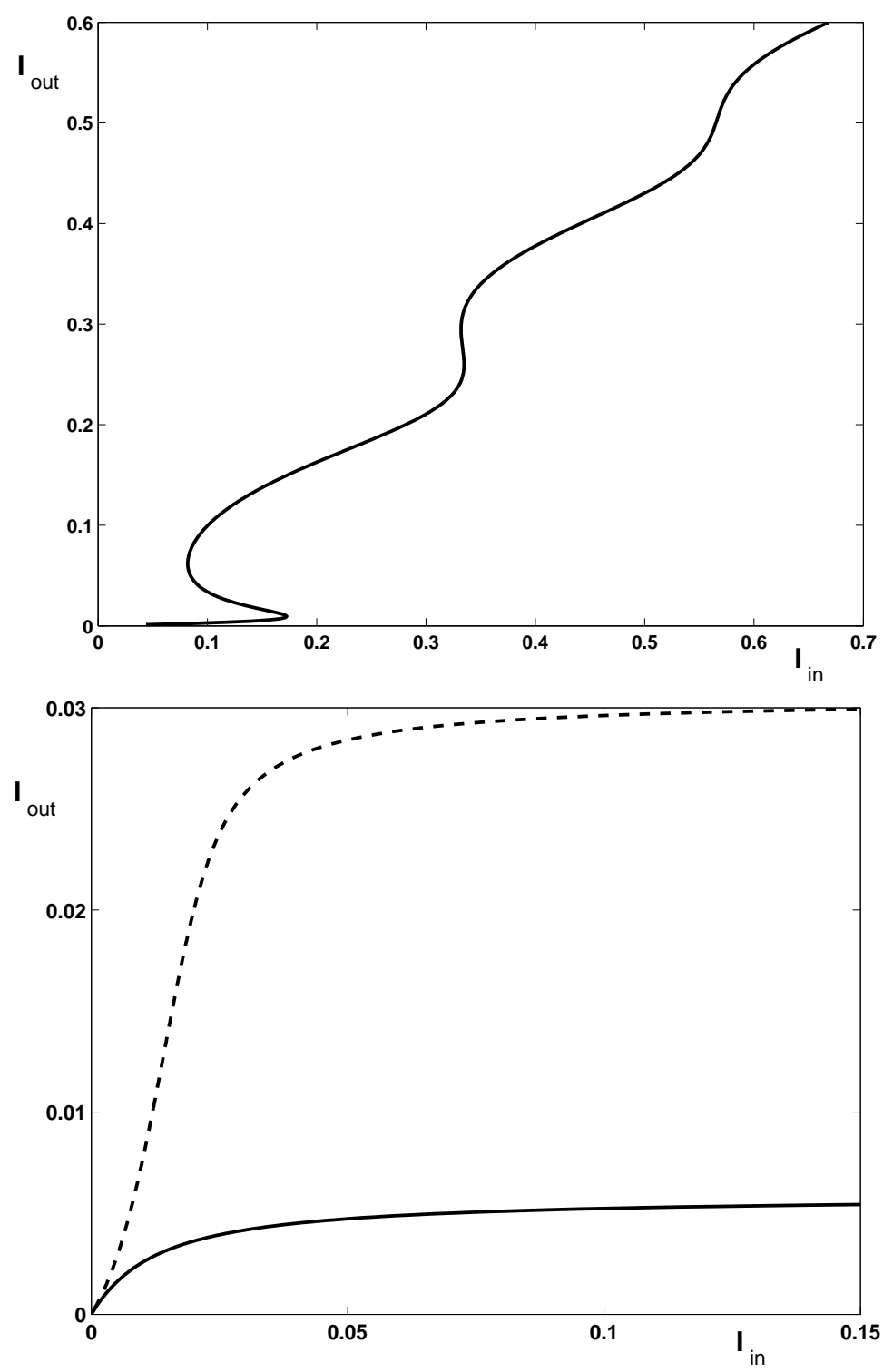

Figure 2: The transmission function $I_{\text {in }}=\mathcal{I}_{L}\left(I_{\text {out }}\right)$ for optical bistability (a) and all-optical limiting (b) in the system (3.11). See parameters of the system in the text. 
origin $a=b=0$, respectively. For the full nonlinear equation, the stable manifold is tangent to the stable (complex) linear subspace spanned by the vector $(1, \mathrm{i})$. By the conservation law (3.6), we check that $|a(z)|^{2}=|b(z)|^{2}=: Q(z)$ for all $z \geq 0$ and we can therefore parameterize the stationary localized solutions as:

$$
a(z)=\sqrt{Q(z)} \mathrm{e}^{\mathrm{i} \theta(z)}, \quad b(z)=\sqrt{Q(z)} \mathrm{e}^{\mathrm{i} \phi(z)} .
$$

Decay in the stable manifold follows the linear decay rate

$$
Q(z)=Q_{\infty} \mathrm{e}^{-2 \delta z}+\mathrm{o}\left(\mathrm{e}^{-2 \delta z}\right)
$$

By phase equivariance, we can obtain the complete set of solutions converging to zero for $z \rightarrow$ $+\infty$ from a single trajectory $\mathbf{A}_{*}(z)$ by simply rotating its phase. By translation invariance, we can shift the solution $\mathbf{A}_{*}(z)$ and find new solutions $\mathbf{A}_{*}\left(z+z_{0}\right)$. Uniqueness of the stable manifold implies that all solutions which decay to zero as $z \rightarrow+\infty$ are of this form. In particular, we can parameterize the set of localized solutions $Q(z)$ by the decay rate of the output intensity $Q_{\infty}$ and the complex phase. Note that the time- $t$ inversion symmetry $a \rightarrow \bar{b}$, $b \rightarrow \bar{a}$ fixes a direction in the stable eigenspace and therefore an orbit in the stable manifold. We may therefore choose $\phi(z)=-\theta(z)$, such that $\lim _{z \rightarrow \infty} \theta(z)=-\frac{\pi}{4}$ as follows from (3.13) and (3.14).

Lemma 3.2 Let $Q_{\infty}$ be the decay rate of the output intensity, as defined in (3.15), which parameterizes the set of localized stationary solutions. Then there exists a maximal decay of the output intensity $0<Q_{\mathrm{lim}} \leq \infty$ and a unique, smooth function $I_{\mathrm{in}}=\mathcal{I}_{\infty}\left(Q_{\infty}\right)$, which is defined for $0 \leq Q_{\infty}<Q_{\text {lim }}$, such that there exists a unique (up to complex phase shift) stationary solution $\mathbf{A}_{*}(z)$ to the system (3.1) with this prescribed decay of the output intensity $Q_{\infty}$ in (3.15). If $Q_{\mathrm{lim}}<\infty$, then the maximum of $Q(z)$ and $I_{\mathrm{in}}$ diverge to infinity as $Q_{\infty} \rightarrow Q_{\mathrm{lim}}$.

Proof. From the discussion above, any localized solution of (3.1) with (3.12) is of the form $\mathbf{A}\left(z ; \varphi, z_{0}\right)=\mathrm{e}^{\mathcal{J} \varphi} \mathbf{A}_{*}\left(z+z_{0}\right)$, generated from a unique solution $\mathbf{A}_{*}(z)$. With the expansions (3.14), (3.15), we have the decay of the output intensity defined as $Q_{\infty}=\mathrm{e}^{2 \delta z_{0}}\left|a_{*}\left(z+z_{0}\right)\right|^{2}$ and the input intensity defined as $I_{\text {in }}=Q(0)=Q_{*}\left(z_{0}\right)$. Since $\mathbf{A}_{*}(z)$ is smooth, we find the smooth dependence of $I_{\text {in }}$ on $\delta z_{0}$ and then on $Q_{\infty}$.

Remark 3.3 The stationary solutions can be parameterized equivalently by $z_{0}$, the shift of solutions in the unstable manifold, or the asymptotic decay rate, which are (monotonically) related by $Q_{\infty}=\mathrm{e}^{-2 \delta z_{0}}$. The advantage of the parameterization by $Q_{\infty}$ is the natural continuous extension to $Q_{\infty}=0$. Since $\mathcal{I}_{\infty}^{\prime}\left(Q_{\infty}\right)=Q^{\prime}(0) Q_{\infty}^{\prime}\left(z_{0}\right)$, the turning point of $I_{\mathrm{in}}=\mathcal{I}_{\infty}\left(Q_{\infty}\right)$ occurs exactly when $Q^{\prime}(0)=0$. 
Again, the stationary localized solution need not be unique for a fixed value of the incident intensity $I_{\text {in }}$. In fact, several stationary localized solutions may exist and have non-monotonically decreasing amplitude $Q(z)$.

As an example, we consider the system (3.1) with the potential $W$ in (1.3). Restricting to $a=\bar{b}$ and exploiting the Hamiltonian function $h$, we find the differential equation [15]

$$
\left(\frac{\mathrm{d} Q}{\mathrm{~d} z}\right)^{2}=Q^{2}\left[4(\delta+2 m Q)^{2}-9 n^{2} Q^{2}\right]
$$

for the localized solution $Q(z)$. The input intensity is given by $I_{\text {in }}=Q(0)$. Two different types of localized solutions may exist in the equation (3.16), as shown on Fig. 3(a,b). The first type on Fig. 3(a) exhibits only one monotonically decreasing solution for a given $I_{\text {in }}$, e.g. for $n=0$, $\delta=0.1, m=5$ (dashed curve) and for $n=0, \delta=0.1, m=-5$ (solid curve). The second type on Fig. 3(b) exhibits two localized solutions for a given $I_{\text {in }}$, e.g. for $n=5, \delta=1, m=0$. One solution is monotonically decreasing (solid curve) and the other solution has a unique maximum (dashed curve). As the decay rate $Q_{\infty}$ tends to infinity, the stationary solution on Fig. 3(b) converges to a reflection-symmetric pulse of the equation (3.16) considered on $z \in \mathbb{R}$, after an appropriate $z$-shift.

Let $\delta>0$. When $\left(16 m^{2}-9 n^{2}\right)>0$ and $m>0$, the localized solution is unique for all values of the input intensity, $0 \leq I_{\text {in }}<\infty$ (see Fig. 3(a), dashed curve). When $n=0$ and $m<0$, the stable manifold connects to a nontrivial equilibrium of (3.1) (see Fig. 3(a), dotted line). Again, the localized solution is unique for any $0<I_{\text {in }}<\frac{\delta}{2|m|}$ (see Fig. 3(a), solid curve). The solution approaches the constant solution when $I_{\text {in }} \rightarrow \frac{\delta}{2|m|}$. For all other parameter values, two stationary solutions coexist for each value of $0<I_{\text {in }}<I_{\text {sol }}$ (see Fig. 3(b), solid and dashed curves). Here $I=I_{\text {sol }}$ is the positive root of the quadratic equation $4(\delta+2 m I)^{2}-9 n^{2} I^{2}=0$

(see Fig. 3(b), dotted line). When $m=0, I_{\text {sol }}=\frac{2 \delta}{3|n|}$. One of the solutions is monotonically decreasing whereas the other possesses a unique maximum at $Q_{\max }=I_{\text {sol }}$. When $I_{\text {in }} \rightarrow I_{\text {sol }}$, the two solutions coalesce in a half-pulse with maximum at $z=0$. The half-pulse corresponds to the stationary solution called the Bragg soliton [20] with amplitude $I_{\text {sol }}$, centered at $z=0$ and restricted to $z \geq 0$. Thus, coexistence of localized solutions occurs precisely when the stable manifold of the origin in the system (3.1) coincides with the unstable manifold to form a homoclinic orbit on $z \in \mathbb{R}$.

\section{Review of optical bistability theory}

Optical bistability in photonic gratings of finite length is the regime, when the equation $I_{\text {in }}=\mathcal{I}_{L}\left(I_{\text {out }}\right)$ has at least two solutions for a given value of the input intensity $I_{\text {in }}$ (see Fig. $2(\mathrm{a}))$. In the physical theory of optical bistability, the branches with negative slopes of $\mathcal{I}_{L}^{\prime}\left(I_{\text {out }}\right)$ 

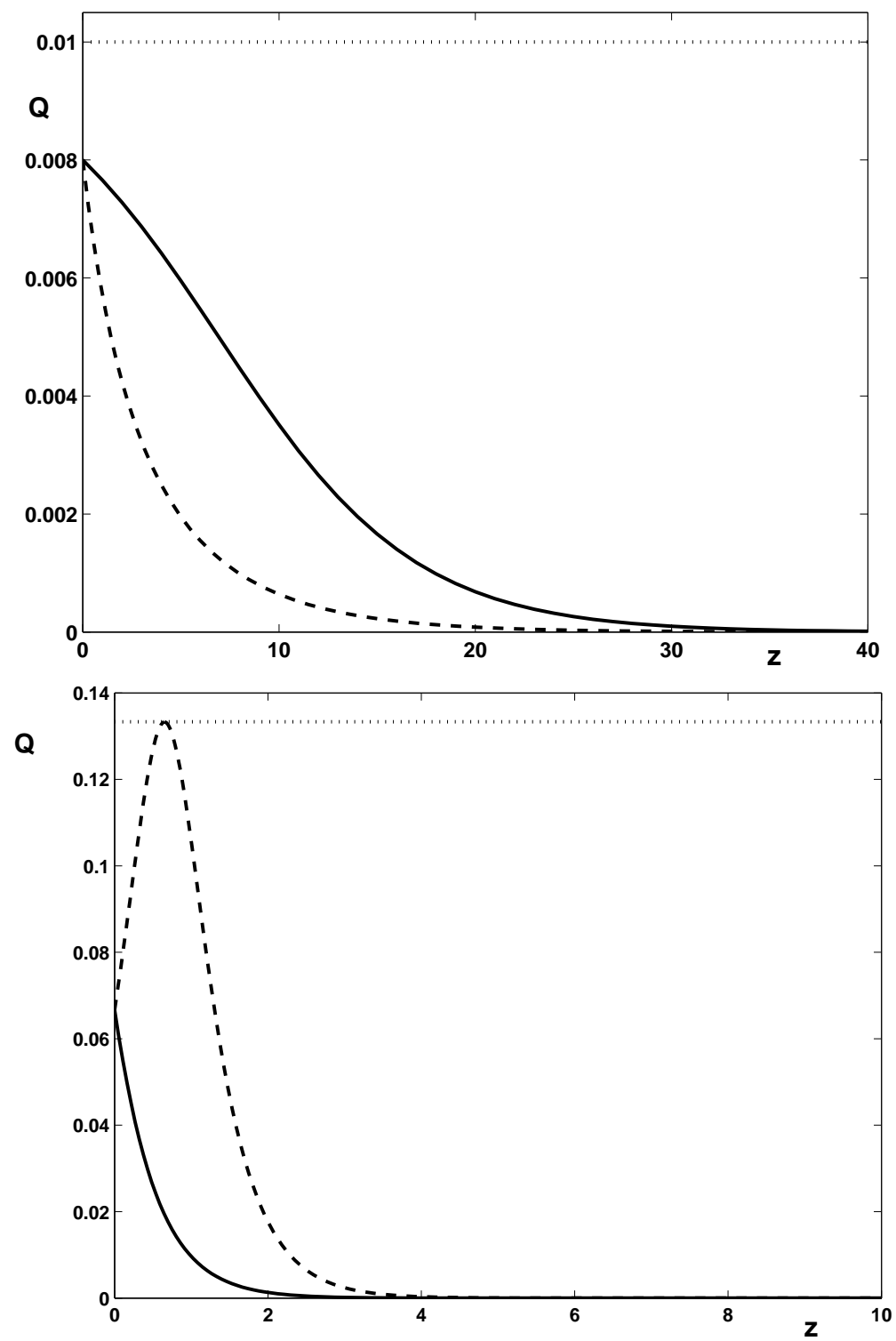

Figure 3: The stationary localized solutions $Q(z)$ of the system (3.16) with single monotonically decreasing solution (a) and with double non-monotonic solutions (b). See parameters of the system in the text. 
are expected to be unstable against small amplitude fluctuations. A physical description of the optical bistability theory is given by Gibbs [5, Appendix E] and by Sterke and Sipe [20, p.223].

A mathematical proof for optical bistability does not seem to be developed, neither for nonlinear Maxwell equations nor for the coupled-mode equations (1.2), although instability in case of negative-slope transmission function $\mathcal{I}_{L}^{\prime}\left(I_{\text {out }}\right)<0$ is generally expected to correspond to unstable eigenvalues of the linearized problem at the stationary solutions. We address this general problem in our analysis in Sections 5-6. Some previous results are listed below.

De Sterke solved the linear stability problem for the system (1.2)-(1.3) with $m=0$ numerically [19]. The numerical shooting method captured a single real unstable eigenvalue for the negative-slope time-independent solutions and a single pair of complex eigenvalues at the upper positive-slope branch of the function $I_{\text {in }}=\mathcal{I}_{L}\left(I_{\text {out }}\right)$.

Ovchinnikov used a direct solution method and solved the linear stability problem for the onedimensional Maxwell equation describing a finite-length uniform nonlinear optical material [10]. A positive unstable eigenvalue was identified for solutions with negative slope of $I_{\text {in }}=$ $\mathcal{I}_{L}\left(I_{\text {out }}\right)$. Complex eigenvalues were also approximated in [10]. Later, Ovchinnikov and Sigal showed that points of zero slope $\mathcal{I}_{L}^{\prime}\left(I_{\text {out }}\right)=0$ are bifurcation points, where an eigenvalue may cross the stability threshold at the origin [11].

Pelinovsky et al. [15] considered the all-optical limiting in the coupled-mode system (1.2)(1.3) for $n=0$ (see Fig. 2(b)). The linear stability problem was analyzed with the use of the AKNS spectral problem [15]. In accordance with the optical bistability theory, the asymptotic stability of all time-independent solutions was proved in the all-optical limiting regime with $n=0$. Numerical finite-difference approximations of unstable eigenvalues were constructed in the general case of $n \neq 0, m \neq 0$ by Pelinovsky and Sargent [14]. One, two, and more real unstable eigenvalues were identified for the negative-slope time-independent solutions after the finite-difference discretization. One, two, and more pairs of complex unstable eigenvalues were found for the upper branches of the positive-slope solutions. Complex eigenvalues were found numerically even for the lowest positive-slope branch in some parameter configurations. We will show in Section 9 that these results are not confirmed by the numerical method based on the Evans function. The additional eigenvalues in [14] are likely to be generated by the coarse finite-difference approximation.

The other major objective of this work is the analysis of spectral stability of localized solutions in semi-infinite and large photonic gratings. To the best of our knowledge, existence and stability of stationary solutions on the semi-infinite interval $z \in[0, \infty)$ have not been considered previously. We show in Section 7 that non-monotonic localized solutions exhibit 
optical bistability similar to stationary solutions in finite-length structures. In particular, the non-monotonic solutions with a positive-slope amplitude at $z=0$ are always spectrally unstable.

\section{$5 \quad$ Stability and instability in finite length structures}

We consider the system (1.2) and (1.4) on the affine space $H_{\text {af }}^{1}(0, L)$, with $L<\infty$. Recall from Section 2 that stability of stationary solutions of $\mathbf{A}_{*}(z)$ is determined by spectral stability of the linearized operator $\mathcal{J} \mathcal{L}_{*}$ whenever no spectrum is located in the closed right half-plane. We therefore consider the linearized equation

$$
\frac{\mathrm{d}}{\mathrm{d} t} \mathbf{A}_{p}=\mathcal{J} \mathcal{L}_{*} \mathbf{A}_{p}
$$

where the operator $\mathcal{J} \mathcal{L}_{*}$ is defined in (2.6), (2.25), (2.26), and (2.27). The perturbation vector $\mathbf{A}_{p}(z, t)$ satisfies the homogeneous boundary conditions (2.28). The linear operator $\mathcal{J} \mathcal{L}_{*}$ for the case $L<\infty$ possesses only isolated eigenvalues of finite multiplicity. In the case $I_{\text {out }}=0$, the spectrum of $\mathcal{J} \mathcal{L}_{*}=\mathcal{J} \mathcal{L}$ is contained in the left half-plane with $\operatorname{Re} \lambda<0$, see Lemma 2.1. The spectrum of $\mathcal{J} \mathcal{L}_{*}$ depends continuously on the solution $\mathbf{A}_{*}(z)$. Therefore, the stationary solution $\mathbf{A}_{*}(z)$ is asymptotically stable for small intensities $I_{\text {out }}$. The goal of this section is to derive an instability criterion based on the slope of $I_{\text {in }}=\mathcal{I}_{L}\left(I_{\text {out }}\right)$, which is the inverse transmission function. We prepare our main result with a necessary criterion for a nontrivial kernel of $\mathcal{L}_{*}$.

The linear eigenvalue problem for $\mathcal{J} \mathcal{L}_{*}$ is

$$
\mathcal{J} \mathcal{L}_{*} \psi=\lambda \psi
$$

where $\boldsymbol{\psi}(z)$ satisfies the homogeneous boundary conditions:

$$
\psi_{1}(0)=\psi_{3}(0)=0, \quad \psi_{2}(L)=\psi_{4}(L)=0
$$

If $\lambda \in \mathbb{C}$, the perturbation vector $\psi(z)$ has no complex conjugation symmetry, i.e. $\psi_{3} \neq \bar{\psi}_{1}$ and $\psi_{4} \neq \bar{\psi}_{2}$, in general.

Lemma 5.1 Define $I_{\text {in }}=\mathcal{I}_{L}\left(I_{\text {out }}\right)$ according to Lemma 3.1 and assume $I_{\text {out }}>0$. Then the operator $\mathcal{J L}_{*}$ is invertible if, and only if, $\mathcal{I}_{L}^{\prime}\left(I_{\text {out }}\right) \neq 0$. When $\mathcal{I}_{L}^{\prime}\left(I_{\text {out }}\right)=0$, the eigenvalue $\lambda=0$ is of geometric multiplicity one.

Proof. Denote by $\mathbf{A}_{*}\left(z ; I_{\text {out }}\right)$ the stationary solution, solving (3.1) with boundary conditions (3.2). We use shooting with the right boundary conditions: $\psi_{2}(L)=\psi_{4}(L)=0$. The subspace 
of solutions to (5.2) satisfying the right boundary condition is complex two-dimensional. The derivative $\boldsymbol{\psi}_{1}:=\partial_{I_{\mathrm{out}}} \mathbf{A}_{*}\left(z ; I_{\mathrm{out}}\right)$ of the family of solutions to the nonlinear equation (3.1) with respect to the boundary value $I_{\text {out }}$ provides one solution to the linear equation (5.2) with $\lambda=0$, satisfying the right boundary condition. Similarly, the derivative $\boldsymbol{\psi}_{2}:=\mathcal{J A}_{*}\left(z ; I_{\text {out }}\right)$ with respect to the family of solutions $\mathrm{e}^{\mathcal{J} \varphi} \mathbf{A}_{*}\left(z ; I_{\text {out }}\right)$, generated by the gauge invariance, provides a second solution to (5.2) with $\lambda=0$ satisfying the right boundary condition. Assuming the condition $\theta(L)=0$ in $(3.10)$, we check that $\psi_{1}(L)=\frac{1}{2 I_{\text {out }}^{1 / 2}}(1,0,1,0)^{T}$ and $\boldsymbol{\psi}_{2}(L)=$ $\mathrm{i} I_{\text {out }}^{1 / 2}(1,0,-1,0)$ and therefore these two solutions are complex linearly independent.

The general solution to (5.2) with $\lambda=0$ satisfying the right boundary conditions is $\boldsymbol{\psi}(z)=$ $c_{1} \psi_{1}(z)+c_{2} \psi_{2}(z)$. The general solution satisfies the left boundary conditions $\psi_{1}(0)=\psi_{3}(0)=$ 0 when a determinant of a linear system for $c_{1}$ and $c_{2}$ is zero, where the determinant is proportional to $\bar{a}(0) \frac{\partial a(0)}{\partial I_{\text {out }}}+a(0) \frac{\partial \bar{a}(0)}{\partial I_{\text {out }}}=\mathcal{I}_{L}^{\prime}\left(I_{\text {out }}\right)$. Since $a(0) \neq 0$, the rank of the coefficient matrix for $c_{1}$ and $c_{2}$ is one if $\mathcal{I}_{L}^{\prime}\left(I_{\text {out }}\right)=0$. Therefore, the kernel of $\mathcal{J} \mathcal{L}_{*}$ is at most onedimensional and is non-empty if $\mathcal{I}_{L}^{\prime}\left(I_{\text {out }}\right)=0$.

Corollary 5.2 When $\mathcal{I}_{L}^{\prime}\left(I_{\text {out }}\right)=0$, the eigenvector $\boldsymbol{\psi}_{0}(z)$ of the kernel of $\mathcal{J} \mathcal{L}_{*}$ is

$$
\boldsymbol{\psi}_{0}(z)=\frac{\partial}{\partial I_{\text {out }}} \mathbf{A}_{*}\left(z ; I_{\text {out }}\right)-\frac{\partial \theta(0)}{\partial I_{\text {out }}} \mathcal{J} \mathbf{A}_{*}\left(z ; I_{\text {out }}\right),
$$

where $\theta(z)$ is the argument of $a(z)$ according to the parameterization (3.10).

The kernel of the adjoint operator is studied in Appendix A. The zero eigenvalue $\lambda$ is algebraically simple if the eigenvectors of $\mathcal{J} \mathcal{L}_{*}$ and its adjoint are not orthogonal to each other. We were not able to prove that the zero eigenvalue is always algebraically simple for the case $L<\infty$. The proofs for the cases $L=\infty$ and $L \gg 1$ are given in Section 7 and Section 8 . However, we show numerically in Section 9 that the zero eigenvalue is simple for all examples considered here.

Whenever $\mathcal{I}_{L}^{\prime}\left(I_{\text {out }}\right) \neq 0$, we define the parity index of the stationary solution $\mathbf{A}_{*}(z)$ as

$$
i\left(\mathbf{A}_{*}\right)=(-1)^{i_{\mathrm{u}}}
$$

where $i_{\mathrm{u}}$ denotes the number of real positive eigenvalues of $\mathcal{J} \mathcal{L}_{*}$, counted with algebraic multiplicity. By compactness of the linearized flow, $\mathcal{E}^{\mathrm{c}} \oplus \mathcal{E}^{\mathrm{u}}$ is finite-dimensional. Therefore, the parity index is well defined. Let us check that the index is constant on a branch of the stationary solution $\mathbf{A}_{*}\left(z ; I_{\text {out }}\right)$, where $\mathcal{I}_{L}^{\prime}\left(I_{\text {out }}\right) \neq 0$. Observe that zero is not an eigenvalue along the branch. Therefore, the only possibility for a change of $i_{\mathrm{u}}$ along such a path is the collision of two complex conjugate eigenvalues on the positive real axis, which does not change the parity $i\left(\mathbf{A}_{*}\right)$. Note that our index is the Leray-Schauder degree of $\left(\exp \left(\mathcal{J} \mathcal{L}_{*} T\right)-\mathrm{id}\right)$, for 
$T$ large enough; see [2] for Leray-Schauder degree theory. Obviously, $i_{\mathrm{u}}=-1$ implies the existence of real positive eigenvalues and the spectral instability of the stationary solution $\mathbf{A}_{*}(z)$.

Proposition 5.3 Suppose the solution curve $I_{\text {in }}=\mathcal{I}_{L}\left(I_{\text {out }}\right)$ has only finitely many turning points $\mathcal{I}_{L}^{\prime}\left(I_{\mathrm{out}}\right)=0$ for $I_{\mathrm{out}} \in\left[0, I_{\mathrm{lim}}\right)$. Then the parity index $i\left(\mathbf{A}_{*}\right)$ is determined by the slope of the input-output transmission function $I_{\text {in }}=\mathcal{I}_{L}\left(I_{\text {out }}\right)$

$$
i\left(\mathbf{A}_{*}\right)=\operatorname{sign} \mathcal{I}_{L}^{\prime}\left(I_{\text {out }}\right)
$$

When $\mathcal{I}_{L}^{\prime}\left(I_{\text {out }}\right)<0$, the stationary solution is spectrally unstable.

Proof. First, notice that small amplitude solutions are stable as is the zero solution for $I_{\text {in }}=I_{\text {out }}=0$. This proves the lemma for small intensities $I_{\text {in }}$ and $I_{\text {out }}$, when $\mathcal{I}_{L}^{\prime}\left(I_{\text {out }}\right)>0$ and $i_{u}=0$. For large intensities $I_{\text {in }}$ and $I_{\text {out }}$, it is sufficient to investigate a point where $\mathcal{I}_{L}^{\prime}\left(I_{\text {out }}\right)=0$ and two branches of stationary solutions collide. At such a collision point, the dynamics can be reduced to a finite-dimensional center manifold; see Proposition 2.7 and Lemma 5.1. The flow is given by a finite-dimensional ordinary differential equation

$$
\dot{u}=f\left(u ; I_{\text {out }}\right) \text {. }
$$

By finite-dimensional degree-theory, we conclude that the parity index $i\left(\mathbf{A}_{*}\right)$ changes sign at any turning point of $I_{\text {in }}=\mathcal{I}_{L}\left(I_{\text {out }}\right)$ as a function of $I_{\text {out }}$.

Remark 5.4 The proof of Proposition 5.3 could be simplified if we could ensure compactness of the time-one map for the nonlinear flow, which would allow for an application of nonlinear Leray-Schauder degree theory, directly.

In the next section, we give yet another way to compute the index, exploiting a variant of the Evans function for the boundary-value problem associated with the linear operator $\mathcal{J} \mathcal{L}_{*}$.

\section{Evans function analysis in finite-length structures}

We present an alternative approach to the instability results reported in Section 5. We exploit the fact that the eigenvalue problem for $\mathcal{J} \mathcal{L}_{*}$ can be written as a system of first-order differential equations:

$$
\frac{\mathrm{d} \boldsymbol{\psi}}{\mathrm{d} z}=[\mathcal{A}(z)+\lambda \mathcal{B}] \boldsymbol{\psi}
$$

The results in this section are similar to those in the previous section. However, we will be able to improve Proposition 5.3 and drop the assumption of finitely many turning points. 
We define a complex analytic function $E_{L}(\lambda)$, called the Evans function, associated with the particular boundary conditions (5.3). The zeroes of the analytic function $E_{L}(\lambda)$ coincide precisely with the eigenvalues $\lambda$ of the linear operator $\mathcal{J} \mathcal{L}_{*}$. The multiplicity of zeroes of the Evans function coincides with the algebraic multiplicity of eigenvalues. The function $E_{L}(\lambda)$ is real for real values of $\lambda$. We will normalize this function such that $E_{L}(\lambda)>0$ for large positive $\lambda$. Note that for any function with these properties, the sign of $E_{L}(0)$ has to coincide with the parity index $i\left(\mathbf{A}_{*}\right)$, defined in Section 5. Indeed, the number of zeroes of the real analytic function on $\lambda \in(0, \infty)$ is even if $E_{L}(0)>0$, and odd if $E_{L}(0)<0$. Again, we have to count zeroes with multiplicity.

We now show how to construct such an analytic function $E_{L}(\lambda)$ for the finite interval $z \in[0, L]$. As a major advantage, this formulation carries over to the case of the unbounded interval $z \in[0, \infty)$, where we loose the compactness, which seems necessary in the construction of the Leray-Schauder type index. As a drawback, the construction is essentially one-dimensional in space $z$.

We define four particular solutions of the system (5.2) on $z \in[0, L]$ with initial conditions

$$
\mathbf{u}_{1}^{-}(0 ; \lambda)=\mathbf{e}_{2}, \quad \mathbf{u}_{2}^{-}(0 ; \lambda)=\mathbf{e}_{4}, \quad \mathbf{u}_{1}^{+}(L ; \lambda)=\mathbf{e}_{1}, \quad \mathbf{u}_{2}^{+}(L ; \lambda)=\mathbf{e}_{3}
$$

where $\mathbf{e}_{j}$ are unit vectors in $\mathbb{R}^{4}$ (note that the solutions $\mathbf{u}_{j}^{ \pm}(z ; \lambda) \in \mathbb{C}^{4}$ will be complex). The two solutions $\left[\mathbf{u}_{1}^{-}(z ; \lambda), \mathbf{u}_{2}^{-}(z ; \lambda)\right]$ span the subspace of solutions satisfying the left boundary conditions (5.3) at $z=0$. The other two solutions $\left[\mathbf{u}_{1}^{+}(z ; \lambda), \mathbf{u}_{2}^{+}(z ; \lambda)\right]$ span the subspace defined by the right boundary conditions (5.3) at $z=L$. The intersection between the two subspaces is traced by the Evans function $E_{L}(\lambda)$ defined as the determinant

$$
E_{L}(\lambda)=-\operatorname{det}\left[\mathbf{u}_{1}^{-}(z ; \lambda), \mathbf{u}_{2}^{-}(z ; \lambda), \mathbf{u}_{1}^{+}(z ; \lambda), \mathbf{u}_{2}^{+}(z ; \lambda)\right] \mathrm{e}^{-2 \lambda L},
$$

where the entire function $\mathrm{e}^{-2 \lambda L}$ is introduced for a normalization of $E_{L}(\lambda)$ for larger positive $\lambda$. Writing $\mathbf{u}_{j}^{ \pm}=\left(u_{j 1}^{ \pm}, u_{j 2}^{ \pm}, u_{j 3}^{ \pm}, u_{j 4}^{ \pm}\right)^{T}$ and taking into account the boundary conditions (6.2) at $z=0$, the $4 \times 4$-determinant in (6.3) reduces to the $2 \times 2$-determinant

$$
E_{L}(\lambda)=\left|\begin{array}{ll}
u_{11}^{+}(0 ; \lambda) & u_{21}^{+}(0 ; \lambda) \\
u_{13}^{+}(0 ; \lambda) & u_{23}^{+}(0 ; \lambda)
\end{array}\right| \mathrm{e}^{-2 \lambda L}
$$

We summarize the properties of the Evans function in the following lemma.

Lemma 6.1 Define the Evans function $E_{L}(\lambda)$ as the determinant in (6.3). Then the Evans function is well-defined, independent of $z$, and is an analytic function of $\lambda \in \mathbb{C}$. Zeros of $E_{L}(\lambda)$ coincide with the spectrum of $\mathcal{J} \mathcal{L}_{*}$ and the multiplicity of zeros of $E_{L}(\lambda)$ corresponds to algebraic multiplicity of eigenvalues of $\mathcal{J L}_{*}$. For real values of $\lambda, E_{L}(\lambda)$ is real and satisfies the normalization condition $E_{L}(\lambda)>0$ for real large positive $\lambda$. 
Proof. The determinant (6.3) is a Wronskian determinant of four particular solutions of a linear system of differential equations (6.1). The volume spanned by these four vectors is invariant under the linear flow since the matrices on the right side of (6.1) all have zero trace.

Since the system of differential equations (6.1) is analytic in $\lambda$, the solutions $\mathbf{u}_{1,2}^{ \pm}(z ; \lambda)$ with the initial values (6.2) are analytic functions of $\lambda$ for any finite $\lambda \in \mathbb{C}$, and so is the determinant $E_{L}(\lambda)$

From the definition, it is clear that $E_{L}(\lambda)$ vanishes precisely when the solutions $\mathbf{u}_{1,2}^{+}(z ; \lambda)$ and $\mathbf{u}_{1,2}^{-}(z ; \lambda)$ are linearly dependent. The system (6.1) then possesses a solution satisfying the boundary conditions (5.3). Following [3], it is straightforward to conclude that the multiplicity of zeroes of $E_{L}(\lambda)$ coincides with the algebraic multiplicity of eigenvalues $\lambda$.

The two equations for $\psi_{3}(z)$ and $\psi_{4}(z)$ in the system (6.1) are complex conjugate to the two equations for $\psi_{1}(z)$ and $\psi_{2}(z)$, with $\lambda$ replaced by $\bar{\lambda}$. This shows that

$$
\begin{aligned}
E_{L}(\lambda) & =-\operatorname{det}\left[\mathbf{u}_{1}^{-}(z ; \lambda), \mathbf{u}_{2}^{-}(z ; \lambda), \mathbf{u}_{1}^{+}(z ; \lambda), \mathbf{u}_{2}^{+}(z ; \lambda)\right] \mathrm{e}^{-2 \lambda L} \\
& =-\operatorname{det}\left[\overline{\mathbf{u}}_{2}^{-}(z ; \bar{\lambda}), \overline{\mathbf{u}}_{1}^{-}(z ; \bar{\lambda}), \overline{\mathbf{u}}_{2}^{+}(z ; \bar{\lambda}), \overline{\mathbf{u}}_{1}^{+}(z ; \bar{\lambda})\right] \mathrm{e}^{-2 \lambda L}=\bar{E}_{L}(\bar{\lambda}) \mathrm{e}^{2(\bar{\lambda}-\lambda) L} .
\end{aligned}
$$

In particular, $E_{L}(\lambda)$ is real for $\lambda \in \mathbb{R}$.

Next, consider the limit $\lambda \rightarrow+\infty, \lambda \in \mathbb{R}$. Set $\lambda=1 / \varepsilon$ and rescale $\zeta=(z-L) / \epsilon$. In the limit $\varepsilon \rightarrow 0$, the problem (6.1) becomes

$$
\begin{aligned}
\frac{\mathrm{d} \psi_{1}}{\mathrm{~d} \zeta}=-\psi_{1}+\mathrm{O}(\varepsilon), & \frac{\mathrm{d} \psi_{3}}{\mathrm{~d} \zeta}=-\psi_{3}+\mathrm{O}(\varepsilon), \\
\frac{\mathrm{d} \psi_{2}}{\mathrm{~d} \zeta}=\psi_{2}+\mathrm{O}(\varepsilon), & \frac{\mathrm{d} \psi_{4}}{\mathrm{~d} \zeta}=\psi_{4}+\mathrm{O}(\varepsilon) .
\end{aligned}
$$

For $\varepsilon=0$, we find explicit solutions of $(6.5)$ as $\mathbf{u}_{1}^{+}(z ; \lambda)=\mathbf{e}_{1} \mathrm{e}^{-\zeta}$ and $\mathbf{u}_{2}^{+}(z ; \lambda)=\mathbf{e}_{3} \mathrm{e}^{-\zeta}$. The formal limit $\varepsilon=0$ in the formula (6.4) gives: $\lim _{\lambda \rightarrow+\infty} E_{L}(\lambda)=\lim _{\varepsilon \rightarrow 0^{+}} E_{L}(\varepsilon)=1$. At $\varepsilon=0$, solutions of (6.5) generate a hyperbolic structure for $\zeta \in \mathbb{R}: \psi_{1,3}(\zeta) \sim \mathrm{e}^{-\zeta}$ and $\psi_{2,4}(\zeta) \sim \mathrm{e}^{\zeta}$. The $\zeta$-dependence of the $\mathrm{O}(\varepsilon)$-terms is slow. For finite $\varepsilon$, the hyperbolic structure persists: there exist unique complex two-dimensional stable and unstable subspaces $\mathcal{E}^{\mathrm{s} / \mathrm{u}}(\zeta)$ such that solutions in $\mathcal{E}^{\mathrm{s} / \mathrm{u}}(\zeta)$ decay exponentially for $\zeta \rightarrow \pm \infty$, respectively. The initial conditions at $\zeta=0$ lie $\mathrm{O}(\varepsilon)$ close to the stable subspace. Transporting the subspace $\left(\psi_{1}, \psi_{2}, \psi_{3}, \psi_{4}\right)=(*, 0, *, 0)$, spanned by these initial conditions, with the linear flow to $\zeta=-L / \varepsilon$, a $\lambda$-Lemma ensures that the subspace is $\mathrm{O}(-c /(\varepsilon L))$-close to the stable subspace $\left(\psi_{1}, \psi_{2}, \psi_{3}, \psi_{4}\right)=(0, *, 0$, *). This ensures that $E_{L}(\lambda)$ is positive, nonzero, for large positive $\lambda$. Note that the function $E_{L}(\lambda)$ therefore cannot vanish entirely and zeroes are therefore isolated. This recovers discreteness of the spectrum of the compact resolvent operator $\mathcal{J} \mathcal{L}_{*}$.

With the Evans function as a tool, we are able to extend Proposition 5.3 to the case of possibly infinitely many turning points. 
Proposition 6.2 The number $i_{\mathrm{u}}$ of real positive eigenvalues $\lambda$ of $\mathcal{J} \mathcal{L}_{*}$ is given by the sign of the derivative of the transmission function $I_{\text {in }}=\mathcal{I}_{L}\left(I_{\text {out }}\right)$ :

$$
\operatorname{sign} \mathcal{I}_{L}^{\prime}\left(I_{\text {out }}\right)=(-1)^{i_{\mathrm{u}}},
$$

whenever $\mathcal{I}_{L}^{\prime}\left(I_{\text {out }}\right) \neq 0$. In particular, the stationary solutions $\mathbf{A}_{*}(z)$ with negative-slope transmission function $\mathcal{I}_{L}^{\prime}\left(I_{\text {out }}\right)<0$ are always spectrally unstable.

Proof. We compute $E_{L}(0)$ in terms of the derivative $\mathcal{I}_{L}^{\prime}\left(I_{\text {out }}\right)$. At $\lambda=0$, the subspace of solutions satisfying the right boundary condition is spanned by

$$
\boldsymbol{\psi}_{1}(z)=\frac{\partial}{\partial I_{\text {out }}} \mathbf{A}_{*}\left(z ; I_{\text {out }}\right), \quad \psi_{2}(z)=\mathcal{J} \mathbf{A}_{*}\left(z ; I_{\text {out }}\right)
$$

The solutions $\mathbf{u}_{1,2}^{+}(z ; \lambda)$ required for the computation of the Evans function are then found explicitly at $\lambda=0$

$$
\begin{aligned}
& \mathbf{u}_{1}^{+}(z ; 0)=\sqrt{I_{\text {out }}}\left[\psi_{1}(z)-\frac{\mathrm{i}}{2 I_{\text {out }}} \psi_{2}(z)\right] \\
& \mathbf{u}_{2}^{+}(z ; 0)=\sqrt{I_{\text {out }}}\left[\boldsymbol{\psi}_{1}(z)+\frac{\mathrm{i}}{2 I_{\text {out }}} \psi_{2}(z)\right]
\end{aligned}
$$

with coefficients determined by the boundary conditions (6.2). A direct computation using (6.4) gives the simple result

$$
E_{L}(0)=\mathcal{I}_{L}^{\prime}\left(I_{\text {out }}\right)
$$

Due to normalization $E_{L}(\lambda)>0$ for large real positive $\lambda$, the real analytic function $E_{L}(\lambda)$, $\lambda \in \mathbb{R}$ possesses an odd number of zeroes in $\lambda>0$ if $\mathcal{I}_{L}^{\prime}\left(I_{\text {out }}\right)<0$.

Corollary 6.3 Assume that the transmission function $I_{\text {in }}=\mathcal{I}_{L}\left(I_{\text {out }}\right)$ only has finitely many extrema. Then the parity index $i\left(\mathbf{A}_{*}\right)$ coincides with the sign of the Evans function evaluated in $\lambda=0$, whenever $\mathcal{I}_{L}^{\prime}\left(I_{\text {out }}\right) \neq 0$

$$
i\left(\mathbf{A}_{*}\right)=\operatorname{sign} E_{L}(0)
$$

Proof. Both quantities, $i\left(\mathbf{A}_{*}\right)$ and $\operatorname{sign} E_{L}(0)$ are nonzero when $\mathcal{I}_{L}^{\prime}\left(I_{\text {out }}\right) \neq 0$ and the kernel is trivial. Also both quantities count the number of eigenvalues on $\operatorname{Re} \lambda>0$ modulo 2 , and therefore coincide.

\section{$7 \quad$ Stability and instability in semi-infinite length structures}

We consider the system (1.2) and (1.5) on the affine space $H_{\mathrm{af}}^{1}(0, \infty)$. We focus on spectral stability which is a necessary criterion for nonlinear stability. A nonlinear stability analysis is beyond the scope of this paper. 
Stationary, localized solutions in semi-infinite structures are described in Lemma 3.2. Spectral stability of such solutions refers to the spectrum of the linear operator $\mathcal{J} \mathcal{L}_{*}$ on $H^{1}\left([0, \infty), \mathbb{C}^{4}\right)$ and boundary conditions (1.5). The corresponding eigenvalue problem is defined by the system $(5.2)$,

$$
\mathcal{J} \mathcal{L}_{*} \psi=\lambda \psi
$$

together with boundary conditions

$$
\psi_{1}(0)=\psi_{3}(0)=0
$$

Since stationary solutions $\mathbf{A}_{*}(z)$ decay to zero as $z \rightarrow+\infty$, the operator $\mathcal{J} \mathcal{L}_{*}$ is a compact perturbation of $\mathcal{J} \mathcal{L}_{\infty}$. Therefore, the essential spectrum is given by $\lambda \in \Gamma_{\infty}$ as defined in Lemma 2.3. Outside of the essential spectrum at $\lambda \in \mathbb{C} \backslash \Gamma_{\infty}$ the eigenvalues $\lambda$ of the point spectrum correspond to exponentially localized eigenfunctions $\psi(z)$ as $z \rightarrow+\infty$. The point spectrum is empty for $I_{\text {in }}=0$.

We emphasize that $\mathcal{J} \mathcal{L}_{*}$ does not possess a compact resolvent and it therefore seems difficult to generalize the Leray-Schauder type reasoning from Section 5 to the semi-infinite interval $L=\infty$. We therefore pursue the approach based on the Evans function construction from Section 6 .

Using exponential dichotomies it is not difficult to see that eigenfunctions actually decay exponentially. Indeed, there is a complex analytic projection $P^{\mathrm{s}}\left(z_{0}\right)$ on the set of initial values at $z=z_{0}$ to bounded solutions of (7.1), with complex two-dimensional range $\mathcal{E}^{\mathrm{s}}\left(z_{0}\right) \subset \mathbb{C}^{4}$. We can choose analytic bases $\mathbf{u}_{1,2}^{+}(z ; \lambda)$ in $\mathcal{E}^{\mathrm{s}}\left(z_{0}\right)$ with prescribed asymptotic behavior:

$$
\lim _{z \rightarrow \infty} \mathrm{e}^{\nu z} \mathbf{u}_{1}^{+}(z ; \lambda)=\frac{1}{\delta}\left(\begin{array}{c}
\delta \\
\mathrm{i}(\nu-\lambda) \\
0 \\
0
\end{array}\right), \quad \lim _{z \rightarrow \infty} \mathrm{e}^{\nu z} \mathbf{u}_{2}^{+}(z ; \lambda)=\frac{1}{\delta}\left(\begin{array}{c}
0 \\
0 \\
\delta \\
\mathrm{i}(\lambda-\nu)
\end{array}\right)
$$

where $\nu=\sqrt{\delta^{2}+\lambda^{2}}$ such that $\operatorname{Re}(\nu)>0$. Note that the square-root is cut precisely along the essential spectrum, where the construction of stable manifolds is ambiguous. We can define two particular solutions of (7.1) through the left boundary condition, just like in the case of $L<\infty$

$$
\mathbf{u}_{1}^{-}(0 ; \lambda)=\mathbf{e}_{2}, \quad \mathbf{u}_{2}^{-}(0 ; \lambda)=\mathbf{e}_{4} .
$$

The two solutions $\left[\mathbf{u}_{1}^{-}(z ; \lambda), \mathbf{u}_{2}^{-}(z ; \lambda)\right]$ span the subspace associated with the left boundary conditions (7.2) at $z=0$ and the other two solutions $\left[\mathbf{u}_{1}^{+}(z ; \lambda), \mathbf{u}_{2}^{+}(z ; \lambda)\right]$ span the two-dimensional subspace $\mathcal{E}^{\mathrm{s}}$ of solutions which remain bounded as $z \rightarrow \infty$. The Evans function $E_{\infty}(\lambda)$ is now 
defined as the determinant

$$
E_{\infty}(\lambda)=-\operatorname{det}\left[\mathbf{u}_{1}^{-}(z ; \lambda), \mathbf{u}_{2}^{-}(z ; \lambda), \mathbf{u}_{1}^{+}(z ; \lambda), \mathbf{u}_{2}^{+}(z ; \lambda)\right]=\left|\begin{array}{cc}
u_{11}^{+}(0 ; \lambda) & u_{21}^{+}(0 ; \lambda) \\
u_{13}^{+}(0 ; \lambda) & u_{23}^{+}(0 ; \lambda)
\end{array}\right|
$$

Again, the Evans function traces intersections between the boundary and stable subspaces and its zeros therefore correspond to the point spectrum of the operator $\mathcal{J} \mathcal{L}_{*}$ with (7.2). We summarize the properties of the Evans function $E_{\infty}(\lambda)$ in the semi-infinite domain $L=\infty$, which are similar to the properties of the Evans function $E_{L}(\lambda)$ in the finite interval $L<\infty$.

Lemma 7.1 Define the Evans function $E_{\infty}(\lambda)$ as the determinant in (7.5). The Evans function is then well-defined, independent of $z$, and is an analytic function in the complement of the essential spectrum $\lambda \in \mathbb{C} \backslash \Gamma_{\infty}$. Zeros of $E_{\infty}(\lambda)$ coincide with point spectrum of $\mathcal{J} \mathcal{L}_{*}$ and multiplicity of zeros corresponds to algebraic multiplicity of eigenvalues. For real values of $\lambda$, $E_{\infty}(\lambda)$ is real and satisfies the normalization condition $E_{L}(\lambda)>0$ for real large positive $\lambda$.

The following proposition is similar to Proposition 6.2. The positive slope of $Q(z)$ at the left boundary $z=0$ plays the role of the negative slope of the transmission function $I_{\text {in }}=\mathcal{I}_{L}\left(I_{\text {out }}\right)$. The amplitude function $Q(z)$ is introduced in the parameterization (3.14) of the stationary localized solution $\mathbf{A}_{*}(z)$.

Proposition 7.2 The number $i_{\mathrm{u}}$ of real positive eigenvalues $\lambda$ of $\mathcal{J} \mathcal{L}_{*}$ is given by the sign of the derivative of the transmission function $I_{\mathrm{in}}=\mathcal{I}_{\infty}\left(Q_{\infty}\right)$ :

$$
\operatorname{sign} \mathcal{I}_{\infty}^{\prime}\left(Q_{\infty}\right)=-\operatorname{sign} Q^{\prime}(0)=(-1)^{i_{\mathrm{u}}}
$$

whenever $\mathcal{I}_{\infty}^{\prime}\left(Q_{\infty}\right) \neq 0$. In particular, the stationary solutions $\mathbf{A}_{*}(z)$ with negative-slope transmission function $\mathcal{I}_{\infty}^{\prime}\left(Q_{\infty}\right)<0$ (corresponding to positive slope $Q^{\prime}(0)>0$ ) are always spectrally unstable.

Proof. We follow the proof of Proposition 6.2. We compute $E_{\infty}(0)$ in terms of $Q^{\prime}(0)$. At $\lambda=0$, the subspace of solutions decaying as $z \rightarrow+\infty$ is spanned by

$$
\boldsymbol{\psi}_{2}(z)=\mathcal{J} \mathbf{A}_{*}(z), \quad \boldsymbol{\psi}_{3}(z)=\mathbf{A}_{*}^{\prime}(z)
$$

The solutions $\mathbf{u}_{1,2}^{+}(z ; \lambda)$ required in the computation of the Evans function are then found explicitly at $\lambda=0$ as

$$
\begin{aligned}
& \mathbf{u}_{1}^{+}(z ; 0)=\frac{-\mathrm{e}^{\frac{\mathrm{i} \pi}{4}}}{2 \delta \sqrt{Q_{\infty}}}\left[\boldsymbol{\psi}_{3}(z)+\mathrm{i} \delta \boldsymbol{\psi}_{2}(z)\right] \\
& \mathbf{u}_{2}^{+}(z ; 0)=\frac{-\mathrm{e}^{-\frac{\mathrm{i} \pi}{4}}}{2 \delta \sqrt{Q_{\infty}}}\left[\boldsymbol{\psi}_{3}(z)-\mathrm{i} \delta \boldsymbol{\psi}_{2}(z)\right]
\end{aligned}
$$


where the coefficients of the linear combinations are found from the boundary conditions (7.3). We evaluate $\mathbf{u}_{1,2}^{+}(z ; 0)$ at $z=0$ and find from (7.5) that

$$
E_{\infty}(0)=\frac{-Q^{\prime}(0)}{2 \delta Q_{\infty}}
$$

This proves the proposition in view of Remark 3.3 and the normalization $E_{\infty}(0)>0$ for large real positive $\lambda$.

Corollary 7.3 When $Q^{\prime}(0)=0, \lambda=0$ is an eigenvalue of $\mathcal{J} \mathcal{L}_{*}$, since $E_{\infty}(0)=0$. The corresponding eigenfunction $\boldsymbol{\psi}_{0}(z)$ can be found explicitly as

$$
\boldsymbol{\psi}_{0}(z)=\mathbf{A}_{*}^{\prime}(z)-\theta^{\prime}(0) \mathcal{J} \mathbf{A}_{*}(z)
$$

where $\theta(z)=\arg (a(z))$ in (3.14).

In the present case of a semi-infinite domain, we can prove that there cannot be any generalized eigenvectors to $\psi_{0}(z)$, i.e. the eigenvalue $\lambda=0$ of the operator $\mathcal{J} \mathcal{L}_{*}$ is always algebraically simple for the case $L=\infty$, when $Q^{\prime}(0)=0$.

To prepare the next lemma, we introduce the $\operatorname{adjoint}(\mathcal{J} \mathcal{L})_{*}^{\text {ad }}$ of the closed and densely defined operator $\mathcal{J L}_{*}$. A direct computation shows that

$$
\left(\mathcal{J} \mathcal{L}_{*}\right)^{\mathrm{ad}}: \mathcal{D}\left(\left(\mathcal{J} \mathcal{L}_{*}\right)^{\mathrm{ad}}\right)=H^{1}\left(\mathbb{R}^{+}, \mathbb{C}^{4}\right) \cap\left\{\phi_{2}(0)=\phi_{4}(0)=0\right\} \rightarrow L^{2}\left(\mathbb{R}^{+}, \mathbb{C}^{4}\right)
$$

Pointwise, the adjoint coincides with $\left(\mathcal{J} \mathcal{L}_{*}\right)^{\text {ad }}=-\mathcal{L}_{*} \mathcal{J}$ and only differs through the adjoint boundary conditions: $\phi_{2}(0)=\phi_{4}(0)=0$ for the adjoint eigenfunction $\phi(z)$. When $Q^{\prime}(0)=$ 0 , the one-dimensional kernel of the adjoint is spanned by a suitable linear combination of $\mathcal{J} \mathbf{u}_{1}^{+}(z ; 0)$ and $\mathcal{J} \mathbf{u}_{2}^{+}(z ; 0)$, namely

$$
\phi_{0}(z)=\mathcal{J} \mathbf{A}_{*}^{\prime}(z)-\theta^{\prime}(0) \mathbf{A}_{*}(z)
$$

Lemma 7.4 The eigenvalue $\lambda=0$ is at most of algebraic multiplicity one.

Proof. Suppose the kernel is nontrivial. We first show that the kernel is at most onedimensional. Shooting with the left initial conditions shows that the kernel is at most twodimensional. The subspace of solutions to the linearized equation which are bounded as $z \rightarrow \infty$ is spanned by $\mathbf{u}_{1}^{+}(z ; 0)$ and $\mathbf{u}_{2}^{+}(z ; 0)$ from (7.7). Inspecting the definition, we see that $\mathbf{u}_{2}^{+}(z ; 0)$ never satisfies the boundary condition $(7.2)$, which shows that the kernel is at most one-dimensional. It remains to show that $\boldsymbol{\psi}_{0}(z)$ does not belong to the range of $\mathcal{J} \mathcal{L}_{*}$. Since we are outside of the essential spectrum, we may use Fredholm's alternative and show that 
the eigenvector $\psi_{0}(z)$ belongs to the range if and only if it is perpendicular to the kernel of the adjoint. Computing the inner product $\left(\boldsymbol{\phi}_{0}, \boldsymbol{\psi}_{0}\right)_{Y}$ in $Y \subset\left[L^{2}(0, \infty)\right]^{4}$, and exploiting that $\mathcal{J}^{\text {ad }}=-\mathcal{J}=\mathcal{J}^{-1}$ is unitary, skew-adjoint, we find

$$
\left(\phi_{0}, \boldsymbol{\psi}_{0}\right)_{Y}=-2 \theta^{\prime}(0) \int_{0}^{\infty} \frac{\mathrm{d}}{\mathrm{d} z}\left(|a|^{2}+|b|^{2}\right) d z=4 \theta^{\prime}(0) Q(0) .
$$

If $Q^{\prime}(0)=0$ and $\theta^{\prime}(0)=0$, then $\mathbf{A}_{*}^{\prime}(0)=0$ and $\mathbf{A}_{*}(z) \equiv$ const, which contradicts the assumption that $\mathbf{A}_{*}(z)$ is the stationary localized solution. Therefore, the eigenfunction $\boldsymbol{\psi}_{0}(z)$ spanning the kernel of $\mathcal{J} \mathcal{L}_{*}$ does not lie in the range and the generalized kernel is one-dimensional as claimed.

We note that the lemma implies that $E_{\infty}^{\prime}(0) \neq 0$ whenever $E_{\infty}(0)=0$. We actually computed the derivatives $E_{\infty}^{\prime}(0)$ and $E_{L}^{\prime}(0)$ for later reference in Appendix B. When $Q^{\prime}(0)=0$ and $E_{\infty}(0)=0$, it follows from (B.12) of Appendix B that

$$
E_{\infty}^{\prime}(0)=\frac{I_{\text {in }}}{\delta Q_{\infty}}(>0)
$$

The sign of $E_{\infty}^{\prime}(0)$ actually gives the direction of crossing for the small eigenvalue near the turning point.

Proposition 7.5 Suppose the stationary solution $\mathbf{A}_{*}(z)$ satisfies the turning point condition $Q^{\prime}(0)=0$ at $I_{\text {in }}=Q(0)=I_{\text {sol }}$. Then, the two stationary localized solutions $\mathbf{A}_{*}(z)$ exist for $I_{\mathrm{in}}<I_{\mathrm{sol}}$ in a local open neighborhood of $I_{\mathrm{sol}}$. The operator $\mathcal{J L}_{*}$ has a small positive eigenvalue $\lambda$ for the branch of solutions with $Q^{\prime}(0)>0$ and a small negative eigenvalue $\lambda$ for the branch of solutions with $Q^{\prime}(0)<0$.

Proof. Consider the Taylor expansion of $E_{\infty}(\lambda)$ near $\lambda=0$ :

$$
E_{\infty}(\lambda)=E_{\infty}(0)+E_{\infty}^{\prime}(0) \lambda+\mathrm{O}\left(\lambda^{2}\right) .
$$

Let $\epsilon=I_{\text {sol }}-I_{\text {in }}>0$ and $Q^{\prime}(0)=\epsilon Q_{1}+\mathrm{O}\left(\epsilon^{2}\right)$. The asymptotic approximation for the eigenvalue $\lambda=\lambda_{0}(\epsilon)$ as zero of $E_{\infty}(\lambda)$ is

$$
\lambda_{0}(\epsilon)=-\frac{E_{\infty}(0)}{E_{\infty}^{\prime}(0)}+\mathrm{O}\left(\epsilon^{2}\right)=\frac{\epsilon Q_{1}}{2 I_{\mathrm{sol}}}+\mathrm{O}\left(\epsilon^{2}\right)
$$

The small eigenvalue $\lambda_{0}(\epsilon)$ is positive for $Q_{1}>0$, i.e. $Q^{\prime}(0)>0$, and it is negative for $Q_{1}<0$, i.e. $Q^{\prime}(0)<0$.

The Evans function $E_{\infty}(\lambda)$ traces eigenvalues in $\operatorname{Re} \lambda>0$ and thereby detects all possible instabilities of stationary localized solutions $\mathbf{A}_{*}(z)$. In order to detect the onset of possible instabilities, it is necessary to extend the Evans function $E_{\infty}(\lambda)$ across the imaginary axis, where the essential spectrum of $\mathcal{J} \mathcal{L}_{*}$ is located, see Lemma 2.3. 
Lemma 7.6 [4, 7] There is $\varepsilon>0$ such that the Evans function $E_{\infty}(\lambda)$ possesses a unique analytic continuation into $\{\operatorname{Re} \lambda>-\varepsilon\} \backslash\{|\operatorname{Im} \lambda|=\delta, \operatorname{Re} \lambda \leq 0\}$. The Evans function $E_{\infty}(\lambda)$ is an analytic function of $\sqrt{\lambda^{2}+\delta^{2}}$ in a neighborhood of $\lambda= \pm \mathrm{i} \delta$. In particular, $E_{\infty}(\lambda)$ is continuous in $\operatorname{Re} \lambda \geq 0$. Moreover, $E_{\infty}(\lambda)$ depends smoothly on $Q_{\infty}$ and is continuous in $Q_{\infty}=0$ as an analytic function of $\lambda$ and $\sqrt{\lambda^{2}+\delta^{2}}$, respectively.

Proof. Analyticity follows from analyticity of the eigenvectors and uniform exponential convergence of the coefficients via a strong-stable manifold argument as in $[4,7]$. Dependence on the decay rate $Q_{\infty}$ is smooth since the coefficients of the linearized equation depend smoothly on $Q_{\infty}$.

Lemma 7.7 For $Q_{\infty}=0$, we have $E_{\infty}(\lambda)>0$ for all $\operatorname{Re} \lambda \geq 0$.

Proof. A straightforward computation shows that the left boundary condition (7.2) does not intersect the eigenspace corresponding to (7.3) in the case $\mathbf{A}_{*}(z) \equiv 0$, for $\operatorname{Re} \lambda \geq 0$, and $\nu=\sqrt{\delta^{2}+\lambda^{2}}$.

We give a physical interpretation of Lemma 7.7. Zeroes of the Evans function on the imaginary axis correspond to radiative modes. The branch point of the Evans function $\lambda=\mathrm{i} \delta$ represents spectrum with a spatially asymptotically constant mode, which possesses zero group velocity. The lemma states that the boundary conditions do not generate either type of modes.

Lemma 7.8 Consider the Evans function depending on $0 \leq Q_{\infty}<Q_{\lim } \leq \infty$. Then there is $M>0$ such that the Evans function does not vanish for $|\lambda|>M$ :

$$
\left|E_{\infty}(\lambda)\right| \geq E_{\infty}>0 \text { for all } \operatorname{Re} \lambda \geq 0,|\lambda| \geq M>0
$$

Proof. The arguments are very similar to [7, Section 2.4] and we omit details.

The previous three lemmas allow us to immediately conclude spectral stability of small amplitude structures in semi-infinite domains.

Corollary 7.9 There is $Q_{*}>0$ such that for all $0 \leq Q_{\infty}<Q_{*}$, the Evans function $E\left(\lambda ; Q_{\infty}\right)$ does not vanish in $\operatorname{Re} \lambda \geq 0$.

\section{Stability and instability in large structures}

The purpose of this section is to bring together the results in the previous three sections and investigate the limit of large structures $L \rightarrow \infty$. This section is organized as follows. We first 
show that the nonlinear stationary bifurcation diagram converges in the limit $L \rightarrow \infty$, Proposition 8.1. We then investigate the behavior of the linearization about a particular stationary solution and show that point spectra of the linearized operator converge in a complement of the essential spectrum of the limiting problem, Proposition 8.2. We then describe the fate of the essential spectrum when truncating the semi-infinite domain. After motivating the results by simple convection-diffusion and scalar coupled-mode equations, we state two results on set-wise convergence of spectra including a neighborhood of the essential spectrum, Propositions 8.4 and 8.6. We also give expansions for the location of eigenvalues approximating the essential spectrum. Together, these results show stability in arbitrarily large structures in the low input intensity regime. We conclude with an expansion for the location of fold points of the inverse transmission function for large domain-size, Proposition 8.9.

We denote by $I_{\text {in }}=\mathcal{I}_{L}\left(I_{\text {out }}\right)$ the (inverse) transmission function in a bounded domain $z \in[0, L]$, Lemma 3.1, and by $I_{\text {in }}=\mathcal{I}_{\infty}\left(Q_{\infty}\right)$ the transmission function in the semi-infinite domain $z \in[0, \infty)$, Lemma 3.2. Recall that $I_{\text {in }}=\mathcal{I}_{L}\left(I_{\text {out }}\right)$ is defined on $I_{\text {out }} \in\left[0, I_{\text {lim }}\right)$ with $I_{\text {lim }} \leq \infty$ and $I_{\text {in }}=\mathcal{I}_{\infty}\left(Q_{\infty}\right)$ is defined on $Q_{\infty} \in\left[0, Q_{\text {lim }}\right)$ with $Q_{\text {lim }} \leq \infty$.

Proposition 8.1 Fix an interval of input intensities $\left\{0 \leq I_{\mathrm{in}} \leq I_{0}\right\}=\mathcal{I}_{\infty}\left(\left[0, I_{+}\right]\right)$such that $\mathcal{I}_{\infty}\left(\left[0, I_{+}\right]\right)$is a compact subset of $\mathcal{I}_{\infty}\left(\left[0, I_{\mathrm{S}}\right)\right)$ and let $L$, the length of the interval be sufficiently large. Then the (inverse) transmission function $\mathcal{I}_{L}\left(I_{\text {out }}\right)$ is defined on $\left[0, I_{+}(L)\right]$ with

$$
I_{+}(L) \geq 2 I_{+} \mathrm{e}^{-\delta L}
$$

The (unique) stationary solution $\mathbf{A}_{*}\left(z ; L, I_{\text {out }}\right)$ corresponding to an output intensity $I_{\text {out }}<$ $I_{+}(L)$ is exponentially close to the stationary solution $\mathbf{A}_{*}\left(z ; \infty, Q_{\infty}\right)$ in the unbounded domain with asymptotic decay rate

$$
Q_{\infty}\left(I_{\text {out }}\right)=\frac{I_{\text {out }}}{2} \mathrm{e}^{-2 \delta L}
$$

such that

$$
\left|\mathbf{A}_{*}\left(z ; L, I_{\text {out }}\right)-\mathbf{A}_{*}\left(z ; \infty, Q_{\infty}\right)\right| \leq C \mathrm{e}^{-\delta L} \mathrm{e}^{-\delta(L-z)} .
$$

Proof. Consider the initial-value problem for the stationary system $(3.1)$ with $a(L)=I_{\text {out }}^{1 / 2}$, $b(L)=0$, such that $I_{\text {out }}$ is small. Since the Hamiltonian system is integrable, the stable manifold of $a=b=0$ possesses a smooth unstable fibration. Projecting the initial value along this fibration onto the stable manifold, we find the following expansion for the solution $\mathbf{A}_{*}\left(z ; L, I_{\text {out }}\right)$ :

$$
\left|\mathbf{A}_{*}\left(z ; L, I_{\text {out }}\right)-\mathrm{e}^{\mathrm{i} \theta} \mathbf{A}_{*}\left(z ; \infty, Q_{\infty}\right)\right|=\mathrm{O}\left(I_{\text {out }} \mathrm{e}^{\delta(z-L)}\right) .
$$

Here, $\mathbf{A}_{*}\left(z ; \infty, Q_{\infty}\right)$ denotes the (unique up to complex phase) solution in the stable manifold with decay $\sqrt{Q_{\infty}} \mathrm{e}^{-\delta z}$. Smoothness of the fibration ensures that the decay rate $Q_{\infty}$ and the 
phase $\theta$ depend smoothly on $I_{\text {out }}>0$. The leading order term is found from the projection of the subspace defined by the right boundary condition along the unstable subspace onto the stable subspace. A straightforward computation gives (8.2) such that the lemma follows from combining (8.4) with the expansion for $I_{\text {out }}$.

We emphasize that the converse of the proposition need not be true: there may exist stationary solutions, which, as $L \rightarrow \infty$, do not converge to a solution in the unbounded domain. As a prototype of such solutions, the reader may think of concatenated pulses, which are forced to remain stationary by the boundary conditions, but, in the semi-infinite domain would interact in time (we actually showed that there cannot be a stationary solution on the semi-unbounded domain consisting of two concatenated copies of the primary pulse, by integrability).

Proposition 8.2 Denote by $\Sigma_{L}$ and $\Sigma_{\infty}$ the spectra of the linearization about $\mathbf{A}_{*}\left(z ; L, I_{\text {out }}\right)$ and $\mathbf{A}_{*}\left(z ; \infty, Q_{\infty}\right)$, described in Proposition 8.1. Then for any compact subset $G$ of the complement of the essential spectrum $\mathbb{C} \backslash \Gamma_{\infty}$, there exists constants $C, \eta>0$ such that

$$
\operatorname{dist}_{\mathrm{H}}\left(\Sigma_{L} \cap G, \Sigma_{\infty} \cap G\right) \leq C \mathrm{e}^{-\eta L},
$$

where dist $_{\mathrm{H}}$ denotes the symmetric Hausdorff distance. If $\lambda_{*} \in \Sigma_{\infty}$, is of algebraic multiplicity $\ell$, then for $L$ sufficiently large there is $\varepsilon>0$ such that there are precisely $\ell$ eigenvalues (counted with multiplicity) in $\Sigma_{L} \cap B_{\varepsilon}\left(\lambda_{*}\right)$, $\varepsilon$ sufficiently small.

Proof. The proof is identical to [17, Lemma 4.3]. Note that the absolute spectrum described there coincides with the essential spectrum due to the reflection symmetry $a \rightarrow b, b \rightarrow a$, $z \rightarrow-z$, which fixes the asymptotic state $a=b=0$.

Corollary 8.3 Let $L>L_{*}$ be sufficiently large. Then the first turning point of the transmission function $\mathcal{I}_{L}^{\prime}\left(I_{\mathrm{out}}\right)=0$ corresponds to a simple eigenvalue of the linearization about the steady state.

If we assume absence of purely imaginary eigenvalues at the turning point, the corollary guarantees existence of a one-dimensional center-manifold, where the dynamics are given by the standard saddle-node $\dot{u}=\mu\left(I_{\text {in }}\right)+u^{2}$, where $u$ parameterizes the kernel (approximately given by the derivative of the half-soliton), and $\mu^{\prime}\left(I_{\text {in }}\right)>0$.

The above results on convergence of point spectrum are complemented by convergence of the essential spectrum. The general setup is similar to [17, Thm $3 \& 5]$. Since the resolvent of the differential operator in a bounded domain is compact, essential spectra disappear. For example, the essential spectrum $(-\infty, 0]$ of $\partial_{z z}$ considered as a closed operator on $L^{2}(\mathbb{R})$ breaks up 
into the countable set $\left\{\frac{n^{2} \pi^{2}}{L^{2}} ; n \in \mathbb{N}\right\}$ when the operator is considered on $z \in[0, L]$ with Dirichlet boundary conditions. Note that as $L \rightarrow \infty$, every individual eigenvalue converges to 0 , but the set of eigenvalues fills out the negative real axis in the limit. Gaps between eigenvalues become small, uniformly in bounded subsets of the complex plane. In particular, point spectra converge to the essential spectrum in the symmetric Hausdorff distance, restricted to compact subsets of the complex plane. In [17], it is shown that this behavior is typical for eigenvalue problems on the real line versus a large finite interval, with asymptotically constant or periodic coefficients, if the essential spectrum is replaced by the absolute spectrum. For example, the spectrum of the convection-diffusion operator $\partial_{z z}+\partial_{z}$ with Dirichlet boundary conditions on $[0, L]$ converges to $(-\infty,-1 / 4]$, and not to the essential spectrum $\lambda=-k^{2}+\mathrm{i} k, k \in \mathbb{R}$. However, whenever the asymptotic problem at $z= \pm \infty$ possesses a reflection symmetry as in our case, essential and absolute spectra coincide.

In order to motivate the following results, we consider the simplest example, where the phenomenon of spectral breakup is visible. Consider the set of two transport equations

$$
u_{t}=u_{x}, \quad v_{t}=-v_{x}
$$

with boundary conditions:

$$
\alpha_{-} u(0)+\beta_{-} v(0)=0, \quad \alpha_{+} u(L)+\beta_{+} v(L)=0 .
$$

Essential and absolute spectra of the operator on the right-hand side, when considered on the entire real axis, coincide with the imaginary axis. The eigenvalue problem in the bounded domain,

$$
\lambda u=u_{x}, \quad \lambda v=-v_{x}, \quad \alpha_{-} u(0)+\beta_{-} v(0)=0, \quad \alpha_{+} u(L)+\beta_{+} v(L)=0,
$$

can be explicitly solved, substituting $u(L)=\mathrm{e}^{\lambda L} u(0), v(L)=\mathrm{e}^{-\lambda L} v(0)$. Eigenvalues correspond to zeroes of the determinant

$$
D(\lambda)=\left(\begin{array}{cc}
\alpha_{-} & \beta_{-} \\
\alpha_{+} \mathrm{e}^{\lambda L} & \beta_{+} \mathrm{e}^{-\lambda L}
\end{array}\right) .
$$

Expanding shows that the equation $D(\lambda)=0$ can be written as a quadratic equation in $\eta=\mathrm{e}^{\lambda L}$,

$$
\alpha_{+} \beta_{-} \eta^{2}-\alpha_{-} \beta_{+}=0
$$

This equation encodes all the scattering information on reflections at the boundary and we call the left-hand-side of (8.9) the scattering polynomial associated with (8.8). The zero $\eta=\eta_{*}$ is given by the average reflection coefficient

$$
\eta_{*}=\sqrt{\frac{\alpha_{-} \beta_{+}}{\beta_{-} \alpha_{+}}} .
$$


In particular, the case $\eta_{*}>1$ gives instability with real positive $\lambda$, while the case $\eta_{*}<1$ gives stability with real negative $\lambda$. The main result in [17] shows that eigenvalue problems for nonlinear systems typically reduce to quadratic polynomial equations of the type (8.9).

When trying to apply the results from [17] to the present setup, we face two major complications. First, the essential spectrum here is double, represented by forward and backward propagating linear waves, together with the complex phase invariance. This violates a "genericity" assumption, Hypothesis 8, in [17]. As a second difficulty, convergence of spectra is not sufficient in order to decide on spectral stability or instability, since the limiting spectrum is located on the (neutrally stable) imaginary axis. We therefore compute the first order correction to the location of the eigenvalues. Since convergence of spectra is different at the edge $\lambda_{*}=\mathrm{i} \delta$ of the essential spectrum, we state the result in this case as a separate proposition. Together, the expansions below allow us to give necessary and sufficient open conditions for spectral stability, uniformly in the size of the domain.

In order to state the result on the asymptotics near the essential spectrum, we have to introduce the equivalent of the scattering polynomial (8.9). Since our eigenvalue problem is posed in $\mathbb{C}^{4}$, and nonautonomous in space $z$, the construction is slightly more involved. However, due to the exponential decay of the stationary solution $\mathbf{A}_{*}(z)$, the linear evolution operator $\varphi_{\infty}(0, L ; \lambda)$ to the system (7.1) is conjugate to the evolution operator $\varphi_{0}(0, L ; \lambda)$ for the constant coefficient system (7.1) with zero potential $W(z) \equiv 0$,

$$
\varphi_{\infty}(0, L ; \lambda)=\tau(\lambda) \varphi_{0}(0, L ; \lambda)+\mathrm{O}\left(\mathrm{e}^{-\delta L}\right) .
$$

We choose a basis $\mathbf{u}_{1,2}^{-}(0 ; \lambda)$ of the linear subspace of $\mathbb{C}^{4}$ which satisfy the boundary conditions (5.3) at $z=0$ and a corresponding basis $\mathbf{u}_{1,2}^{+}(L ; \lambda)$ for $z=L$, see (6.2). Eigenvalues correspond to zeros of the determinant:

$$
D(\lambda)=\operatorname{det}\left(\tau(\lambda) \varphi_{0}(0, L ; \lambda) \mathbf{u}_{1}^{-}(0 ; \lambda), \tau(\lambda) \varphi_{0}(0, L ; \lambda) \mathbf{u}_{2}^{-}(0 ; \lambda), \mathbf{u}_{1}^{+}(L ; \lambda), \mathbf{u}_{2}^{+}(L ; \lambda)\right)
$$

We freeze $\tau(\lambda)$ at a given value $\lambda=\lambda_{*}$. Expliciting the determinant $D(\lambda)$, we find the fourth order scattering polynomial

$$
c_{4}\left(\lambda_{*}\right) \eta^{4}+c_{2}\left(\lambda_{*}\right) \eta^{2}+c_{0}\left(\lambda_{*}\right)=0, \quad \eta=\mathrm{e}^{2 \nu(\lambda) L},
$$

where $\nu(\lambda)=\sqrt{\delta^{2}+\lambda^{2}}$.

Proposition 8.4 Assume that for some $\lambda_{*} \in \mathrm{i}(\delta, \infty)$, the scattering polynomial (8.11) possesses a simple root $\eta=\eta_{*} \neq 0$. Then, in the setting of Proposition 8.2, the point spectra in the bounded domain converge to the union of essential and point spectrum in the unbounded domain,

$$
\operatorname{dist}_{\mathrm{H}}\left(\Sigma_{L} \cap G, \Sigma_{\infty} \cap G\right) \rightarrow 0 \text { as } L \rightarrow \infty
$$


Here dist $_{\mathrm{H}}$ denotes the symmetric Hausdorff distance and $G$ any compact subset of the complex plane. In particular, the number of eigenvalues in a small neighborhood of a point $\lambda_{*}$ of the essential spectrum converges to infinity as $L \rightarrow \infty$.

Moreover, if all four roots of (8.11) satisfy $\left|\eta_{*}\right|<1$, then all eigenvalues in a neighborhood of $\lambda_{*}$ possess negative real part for $L$ sufficiently large. If there exists a root with $\left|\eta_{*}\right|>1$, then there exist unstable eigenvalues in a neighborhood of $\lambda_{*}$ for $L$ sufficiently large.

Proof. Choose a point $\lambda_{*}=\mathrm{i} \omega$ with $\omega>\delta$ such that $\eta_{*}$ is a simple root of the scattering polynomial (8.11). We show that in any fixed neighborhood of this point, the number of eigenvalues tends to infinity. Denote by $\mathcal{E}_{\mp} \subset \mathbb{C}^{4}$ the subspaces corresponding to the left and right boundary conditions (5.3). Let $\varphi_{\infty}(z, \zeta ; \lambda)$ and $\varphi_{L}(z, \zeta ; \lambda)$ denote the linear evolution operator on $\mathbb{C}^{4}$ to $(7.1)$ and (5.2), with potential $W(z)$ evaluated for the stationary solutions $\mathbf{A}_{*}(z)$ in the semi-infinite and unbounded structure, respectively. Since the nonlinear stationary states converge exponentially to the stationary state in the semi-infinite structure (8.3), we have $\left|\varphi_{\infty}(z, \zeta ; \lambda)-\varphi_{L}(z, \zeta ; \lambda)\right| \leq C \mathrm{e}^{-\delta L}$, uniformly in $0 \leq z, \zeta \leq L$ and $\lambda$ close to $\lambda_{*}$.

Denote by $\mathcal{E}^{\mathrm{s}}(z ; \lambda) \subset \mathbb{C}^{4}$ the stable subspace of initial values $\boldsymbol{\psi}(z)$ at $z=0$ such that $\left|\varphi_{\infty}(\zeta, z ; \lambda) \psi(z)\right| \rightarrow 0$ as $\zeta \rightarrow \infty$. From Lemma 7.6 and its proof we can conclude that $\mathcal{E}^{\mathrm{s}}(z ; \lambda) \subset \mathbb{C}^{4}$ is analytic in $\lambda$ in a neighborhood of $\lambda_{*}$.

Eigenvalues in the bounded domain $[0, L]$ correspond to nontrivial intersections between the subspaces $\varphi_{L}(0, L ; \lambda) \mathcal{E}_{-}$with $\mathcal{E}_{+}$.

Let $\varphi_{0}(z, \zeta ; \lambda)$ denote the solution operator to $(7.1)$ with zero potential $W(z) \equiv 0$. Exponential decay of the stationary solution implies that there exists a linear invertible transformation $\tau(\lambda)$, analytic in $\lambda$, such that

$$
\left|\varphi_{\infty}(0, L ; \lambda)-\tau(\lambda) \varphi_{0}(0, L ; \lambda)\right| \leq C \mathrm{e}^{-\delta L}
$$

In particular $\varphi_{\infty}(0, L ; \lambda) \mathcal{E}_{-}$is exponentially close to $\tau(\lambda) \varphi_{0}(0, L ; \lambda) \mathcal{E}_{+}$as a linear subspace of $\mathbb{C}^{4}$. The linear transformation $\tau(\lambda)$ measures the influence of the $z$-dependence of the coefficient. Note that $\tau(\lambda)$ is independent of $L$. It is therefore sufficient to investigate nontrivial intersections between the subspaces $\tau(\lambda) \varphi_{\infty}(0, L ; \lambda) \mathcal{E}_{-}$and $\mathcal{E}_{+}$. The linear evolution $\varphi_{0}(0, L ; \lambda)$ is explicitly known from $(2.13)$. We next choose bases in $\mathcal{E}_{ \pm}$and compute $D(\lambda)$ as in (8.10). Again, $D(\lambda)$ vanishes precisely when $\lambda$ is an eigenvalue. Exploiting the explicit form of $\varphi_{0}(0, L ; \lambda)$, we find that

$$
D(\lambda)=c_{4}(\lambda) \mathrm{e}^{2 \nu(\lambda) L}+c_{2}(\lambda)+c_{0}(\lambda) \mathrm{e}^{-2 \nu(\lambda) L}
$$

with analytic coefficients $c_{0,2,4}(\lambda)$. We may multiply by $\mathrm{e}^{2 \nu(\lambda) L} \neq 0$ and substitute $\eta=$ 
$\mathrm{e}^{2 \nu(\lambda) L} \neq 0$ to arrive at the eigenvalue equation

$$
c_{4}(\lambda) \eta^{4}+c_{2}(\lambda) \eta^{2}+c_{0}(\lambda)=0
$$

Since $\eta^{\prime}(\lambda)$ is large, of order $\mathrm{O}(L)$, we first study the system with frozen coefficients $c_{0,2,4}(\lambda)$ at $\lambda=\lambda_{*}$. We arrive at the scattering polynomial (8.11), which possesses a nonzero simple root $\eta=\eta_{*}$, by assumption. We may therefore locally solve (8.14) with $\eta=\tilde{\eta}(\lambda)$ near $\lambda=\lambda_{*}$, $\eta=\eta_{*}$. Substituting into the relation between $\eta$ and $\lambda$ gives

$$
\mathrm{e}^{\nu(\lambda) L}= \pm \tilde{\eta}(\lambda)
$$

Since $\nu^{\prime}\left(\lambda_{*}\right) \neq 0$, we may as well consider $\nu$ as the independent variable

$$
\mathrm{e}^{\nu L}= \pm \eta(\nu)
$$

where $\eta(\nu)=\tilde{\eta}(\lambda(\nu))$. Expanding $\nu=\mathrm{i} k_{*}+\rho / L$ gives

$$
\mathrm{e}^{\rho}= \pm \mathrm{e}^{-\mathrm{i} k_{*} L}\left(\eta_{*}+\mathrm{O}\left(\rho L^{-1}\right)\right) .
$$

Neglecting the error term, which is small together with its derivatives, we find infinitely many solutions $\rho_{*}^{ \pm}+2 \pi \mathrm{i} \ell, \ell \in \mathbb{Z}$. For each fixed $\ell_{0}$, a winding number argument shows that for $L$ large enough, all solutions with $|\ell| \leq \ell_{0}$ persist for the full equation with error terms. This proves the proposition when $\eta_{*}$ is a simple zero.

By analyticity, the set of $\lambda_{*}$ such that $\eta_{*} \neq 0$ is a simple zero of (8.11) is dense. This shows that almost every point in the essential spectrum is an accumulation point. By compactness, the spectra inside $G$ then converge in the symmetric Hausdorff distance to a compact set, which has to coincide with the union of the essential and point spectra inside $G$.

Note that

$$
\operatorname{Re} \nu=\operatorname{Re} \rho^{ \pm}=\frac{1}{L} \log \left|\eta_{*}\right|+\mathrm{O}\left(L^{-2}\right),
$$

such that $\operatorname{sign}(\operatorname{Re} \lambda)=\operatorname{sign}(\operatorname{Re} \nu)<0$ if $\left|\eta_{*}\right|<1$ and $\operatorname{sign}(\operatorname{Re} \lambda)>0$ if $\left|\eta_{*}\right|>1$, which proofs the claims on stability.

Corollary 8.5 The conclusions of Proposition 8.4 hold for all but possibly finitely many values of the decay rate $Q_{\infty}$ if the potential $W$ is an analytic function on $\mathbb{C}^{4}$.

Proof. The coefficients $c_{0,2,4}(\lambda)$ of the scattering polynomial $D(\lambda)$ in (8.13) are analytic functions of $\lambda$. For $Q_{\infty}=0$, the roots are disjoint and nonzero. Therefore, multiple roots or vanishing of $c_{4}(\lambda)$ occurs for at most a discrete set of values of $Q_{\infty}$. 
We emphasize that most (all but finitely many) eigenvalues $\lambda$ for the linearization in a bounded domain with $L<\infty$ typically converge to i $\delta$ (or $-\mathrm{i} \delta$ ), which is the edge of the essential spectrum in the unbounded domain $L=\infty$. Still, multiplicity in a neighborhood of any fixed point of the essential spectrum goes to infinity because of new eigenvalues coming in from infinity as $L \rightarrow \infty$.

We next address the asymptotics of the spectrum near the double root $\lambda=\mathrm{i} \delta$ of the dispersion relation. Going through the construction of the scattering polynomial (8.11) at $\lambda_{*}=\mathrm{i} \delta$, and substituting the explicit form of the linear evolution $\varphi_{0}(0, L ; \lambda)$ with zero potential $W(z) \equiv 0$, we find the scattering polynomial in the form

$$
c_{2}\left(\lambda_{*}\right) \eta^{2}+c_{1}\left(\lambda_{*}\right) \eta+c_{0}\left(\lambda_{*}\right)=0, \quad \eta=\frac{\tanh (\nu L)}{\nu} .
$$

Proposition 8.6 Assume that the scattering polynomial (8.16) at the edge $\lambda_{*}=\mathrm{i} \delta$ of the essential spectrum possesses two simple roots $\eta_{1,2} \neq 0$. Then, the spectrum in a neighborhood of $\lambda_{*}=\mathrm{i} \delta$ satisfies the expansion

$$
\lambda_{\ell, j}=\mathrm{i} \delta+2 \pi^{2} \mathrm{i} \frac{\ell^{2}}{L^{2} \delta}\left(1+\frac{2 \eta_{j}}{L}+\mathrm{O}\left(L^{-2}\right)\right), \quad j=1,2, \quad \ell=1,2,3, \ldots
$$

In particular, all eigenvalues in a neighborhood of $\lambda_{*}$ are stable if $\operatorname{Im} \eta_{1,2}>0$ and $L$ is sufficiently large. If $\operatorname{Im} \eta_{1}<0$ or $\operatorname{Im} \eta_{2}<0$, then there exist unstable eigenvalues $\lambda$ for sufficiently large $L$.

Proof. The proof is similar to the proof of Proposition 8.4. Eigenvalues are zeros of the determinant $D(\lambda)$ in (8.10). Near the double root $\lambda=\lambda_{*}$ of the dispersion relation, the evolution operator $\varphi_{0}(0, L ; \lambda)$ is generated by $\cosh (\nu L)$ and $\sinh (\nu L) / \nu$ as in $(2.13)$ such that we derive the expansion

$$
D(\lambda)=c_{0}\left(\lambda_{*}\right) \cosh ^{2}\left(\nu\left(\lambda_{*}\right) L\right)+c_{1}\left(\lambda_{*}\right) \cosh \left(\nu\left(\lambda_{*}\right) L\right) \frac{\sinh \left(\nu\left(\lambda_{*}\right) L\right)}{\nu\left(\lambda_{*}\right)}+c_{2}\left(\lambda_{*}\right) \frac{\sinh ^{2}\left(\nu\left(\lambda_{*}\right) L\right)}{\nu^{2}\left(\lambda_{*}\right)} .
$$

Dividing by $\cosh ^{2}\left(\nu\left(\lambda_{*}\right) L\right)$, we arrive at the scattering polynomial (8.16) at the edge of the essential spectrum $\lambda_{*}=\mathrm{i} \delta$. By assumption, there are two distinct zeroes of (8.16), such that $\eta_{1 / 2} \neq 0$. We may therefore solve (8.16) for $\eta=\eta_{j}(\nu)$ and substitute into

$$
\frac{\tanh (\nu L)}{\nu L}=\frac{\eta(\nu)}{L}
$$

Solutions are located close to $\nu L=2 \pi \mathrm{i} \mathbb{Z}$. We therefore expand $\nu L=2 \pi \mathrm{i} L+y$ and solve for $y$ close to zero

$$
y=\frac{2 \pi \mathrm{i} \ell \eta_{j}}{L}+\mathrm{O}\left(L^{-2}\right), \quad \ell \in \mathbb{Z}
$$


Substituting this expansion into the expression for $\lambda$ as a function of $\nu$, we find

$$
\lambda=\mathrm{i} \delta+\frac{\nu^{2}}{2 \mathrm{i} \delta}+\mathrm{O}\left(\nu^{3}\right)=\mathrm{i} \delta+2 \pi^{2} \mathrm{i} \frac{\ell^{2}}{L^{2} \delta}\left(1+\frac{2 \eta_{j}}{L}+\mathrm{O}\left(L^{-2}\right)\right) .
$$

This proves the proposition.

The results until now show that existence and stability properties are "convergent" in the limit $L \rightarrow \infty$. In particular, unstable stationary solutions in the semi-infinite domain are approximated by unstable solutions in large bounded intervals. In the semi-infinite domain, stability of the stationary solutions is always understood as marginal stability since the essential spectrum is located on the imaginary axis, which marks the stability threshold. The previous propositions give necessary criteria, when marginal stability in the unbounded domain induces stability in the bounded domain.

Corollary 8.7 Assume that the stability criteria of Propositions 8.4 and 8.6 are met. In particular, assume that the roots $\eta_{*}$ of (8.11) are disjoint and of modulus less than 1 for all $\lambda$ in the essential spectrum, and assume that the roots $\eta_{1,2}$ of (8.16) are disjoint and possess strictly positive imaginary part. Moreover, assume that there is no point spectrum in $\{\lambda \in \mathbb{C} ; \operatorname{Re} \lambda \geq 0\}$. Then the family of stationary solutions in the domain $[0, L]$ approximating the stationary solutions in the semi-infinite domain is asymptotically stable for sufficiently large $L$.

Proof. From Propositions 8.4 and 8.6, we conclude that the essential spectrum is contained in the left half-plane of $\lambda$ for bounded imaginary parts. On the other hand, for each $L$ there exists $M(L)>0$ such that any unstable eigenvalue $\lambda$ possesses imaginary part bounded by $M(L)$. The only remaining possibility for unstable eigenvalues is a sequence $\lambda\left(L_{k}\right)$ with $\operatorname{Im} \lambda\left(L_{k}\right) \rightarrow \infty, \operatorname{Re} \lambda\left(L_{k}\right)>0$. We claim, however that there is $M>0$ such that there is no spectrum in $\{|\operatorname{Im} \lambda|>M\} \cap\{\operatorname{Re} \lambda \geq 0\}$, independent of $L$. To see this, we note that the Evans function $E_{\infty}(\lambda)$ in the unbounded domain can be extended across the imaginary axis and does not vanish for large imaginary parts of $\lambda$, Lemma 7.8. Since the Evans function $E_{L}(\lambda)$ in bounded domains is $\mathrm{e}^{-\delta L}$-close to the Evans function $E_{\infty}(\lambda)$ in the unbounded domain, we can exclude unstable spectrum with large imaginary part, uniformly in the size $L \geq L_{0}$ of the domain.

Corollary 8.8 There exists $I_{*}>0$ such that all stationary solutions $\mathbf{A}_{*}(z)$ with parameter $I_{\text {in }}$ in the domain $0 \leq I_{\text {in }}<I_{*}$ are asymptotically stable, for any size $L<\infty$ of the domain.

We expect zeroes of the Evans function $E_{\infty}(\lambda)$ on the imaginary axis to be non-generic in the parameter domain $0 \leq I_{\text {in }}<I_{*}$. There is one particular case, where a zero on the imaginary 
axis can be computed, namely when the parameter $I_{\text {in }}$ equals the maximal amplitude $I_{\text {sol }}$ of the pulse (Bragg soliton) solution. We already noticed in Corollary 8.3 that the turning point of $I_{\text {in }}=\mathcal{I}_{\infty}\left(Q_{\infty}\right)$ persists in finite structures $L<\infty$. We now give an expansion for the location of the turning point of $I_{\text {in }}=\mathcal{I}_{L}\left(I_{\text {out }}\right)$.

Proposition 8.9 Let $I_{\text {sol }}$ denote the input intensity at the turning point of the transmission function $\mathcal{I}_{\infty}\left(Q_{\infty}\right)$ and denote the corresponding stationary solution by $\mathbf{A}_{\mathrm{sol}}(z)$. Then the transmission function for the finite-length structure possesses a unique turning point close to $I_{\text {sol }}$ with maximal input intensity

$$
I_{\text {in }}=I_{\text {sol }}+4 Q_{\infty} \mathrm{e}^{-2 \delta L}+\mathrm{o}\left(\mathrm{e}^{-2 \delta L}\right) .
$$

Proof. For convenience, we assume that $a=\bar{b}$, thus fixing the argument of $a$ and $b$ such that $\theta(z)=-\phi(z)$ in (3.14). Together with the linearized equation (7.1) and (7.2), we consider the adjoint problem (7.10) at the turning point $I_{\text {sol }}$. Since $h$ and $I_{\mathrm{pt}}$ are conserved quantities, $\nabla h$ and $\nabla I_{\mathrm{pt}}$ evaluated in $\mathbf{A}_{\text {sol }}$ are eigenfunctions of $\mathcal{J} \mathcal{L}_{*}$. We therefore define

$$
\begin{aligned}
\phi_{1}(z) & =\nabla h\left(\mathbf{A}_{\mathrm{sol}}(z)\right)=\left(\mathrm{i} a^{\prime}(z),-\mathrm{i} b^{\prime}(z),-\mathrm{i} \bar{a}^{\prime}(z), \mathrm{i} \bar{b}^{\prime}(z)\right)^{T} \\
\phi_{2}(z) & =\frac{1}{2} \nabla I_{\mathrm{pt}}\left(\mathbf{A}_{\mathrm{sol}}(z)\right)=(a(z),-b(z), \bar{a}(z),-\bar{b}(z))^{T} .
\end{aligned}
$$

To leading order, the perturbation of the profile in the semi-infinite domain solves the linearized equation (7.1). The scalar product of solutions to this equation with solutions to the adjoint variational equation is preserved. In order to find the correction in the amplitude of the stationary solution, we have to find the projection of the solution to the linearized equation on $(a(0), 0, \bar{a}(0), 0)$. The corresponding solution to the adjoint variational equation is

$$
\phi(z)=\phi_{1}(z)+\beta \phi_{2}(z), \quad \beta=-\mathrm{i} \frac{b^{\prime}(0) \bar{b}(0)}{b(0) \bar{b}(0)} \in \mathbb{R},
$$

such that

$$
\phi(0)=\left(2 \mathrm{i} a^{\prime}(0), 0,-2 \bar{i}^{\prime}(0), 0\right)^{T} .
$$

Here, we used repeatedly $a(z)=\bar{b}(z)$ and $\operatorname{Re}\left(a^{\prime}(0) \bar{a}(0)\right)=\operatorname{Re}\left(b^{\prime}(0) \bar{b}(0)\right)=0$ since the amplitude is maximal in $z=0$, by assumption. Note that $\mathrm{i} a^{\prime}(0) a(0)<0$ such that $\phi(0)$ indicates the direction of decreasing input intensity.

The stationary solution $\mathbf{A}_{*}(z)$ for finite $L<\infty$ possesses the expansion,

$$
\mathbf{A}_{*}(L)=(1-\mathrm{i}, 0,1+\mathrm{i}, 0)^{T} \sqrt{2 Q_{\infty}} \mathrm{e}^{-\delta L}+\mathrm{o}\left(\mathrm{e}^{-\delta L}\right) .
$$

The correction to the input intensity $\Delta I_{\text {in }}$ at the fold point is therefore given to leading order by a scalar product

$$
\sqrt{\Delta I_{\text {in }}}=-\left(\phi(L), \mathbf{A}_{*}(L)\right)_{X}+\mathrm{o}\left(\mathrm{e}^{-2 \delta L}\right) .
$$


In order to evaluate the scalar product, we use the explicit representations (8.20) and (8.21) and derive:

$$
\sqrt{\Delta I_{\text {in }}}=2 \operatorname{Re}\left[-\mathrm{i}\left(a^{\prime}(L)-\frac{\bar{a}^{\prime}(0) a(0)}{a(0) \bar{a}(0)} a(L)\right)(1-\mathrm{i}) \sqrt{2 Q_{\infty}} \mathrm{e}^{-\delta L}\right]+\mathrm{o}\left(\mathrm{e}^{-2 \delta L}\right) .
$$

Expanding

$$
a(L)=\left((1-\mathrm{i}) \sqrt{Q_{\infty} / 2}+\mathrm{o}(1)\right) \mathrm{e}^{-\delta L}, \quad a^{\prime}(L)=-\delta\left((1-\mathrm{i}) \sqrt{Q_{\infty} / 2}+\mathrm{o}(1)\right) \mathrm{e}^{-\delta L},
$$

we arrive at

$$
\sqrt{\Delta I_{\text {in }}}=4 Q_{\infty} \mathrm{e}^{-2 \delta L}+\mathrm{o}\left(\mathrm{e}^{-2 \delta L}\right)
$$

This proves the proposition.

\section{$9 \quad$ Example of cubic nonlinearities}

We analyze the example of the system (1.2) with the potential function $W$ in (1.3). We prove analytically that the stationary solutions are spectrally stable in the case $n=0$. Then, we study numerically the unstable real and complex eigenvalues of the spectral problem in the case $m=0$.

Case $n=0$ : The transmission function $I_{\text {in }}=\mathcal{I}_{L}\left(I_{\text {out }}\right)$ is shown on Fig. 2(b) for $m>0$ (solid curve) and for $m<0$ (dashed curve). It displays all-optical limiting, such that no turning points exist, where $\mathcal{I}_{L}^{\prime}\left(I_{\text {out }}\right)=0$. Spectral stability can not be deduced from the parity index analysis of the present paper, since unstable complex eigenvalues or an even number of positive eigenvalues might exist for the linearized problem with monotonically increasing transmission characteristic $I_{\text {in }}=\mathcal{I}_{L}\left(I_{\text {out }}\right)$. Nevertheless, we prove spectral stability of the stationary solutions $\mathbf{A}_{*}(z)$ in the case $n=0$ by a direct method. Recall the normalization $\delta \geq 0$.

Proposition 9.1 Let $\mathbf{A}_{*}(z)$ be the stationary solution of the system (3.1) on $z \in[0, L]$ with boundary conditions (3.2) and with potential $W$ in (1.3) with $n=0, m>0$. The spectrum of the linear operator $\mathcal{J} \mathcal{L}_{*}$ is located in the open left-half plane $\{\lambda: \operatorname{Re} \lambda<0\}$, that is, all stationary solutions $\mathbf{A}_{*}(z)$ are unique (for a fixed input intensity $I_{\text {in }}$ ) and asymptotically stable.

Proof. The stationary solutions $\mathbf{A}_{*}(z)$ are parameterized in $(3.10)$ with $\theta(z) \equiv 0$ and $\phi(z) \equiv \pi / 2$, or explicitly

$$
a(z)=\sqrt{I_{\text {out }}+Q(z)}, \quad b(z)=\mathrm{i} \sqrt{Q(z)}
$$


where $Q(z)$ solves the first-order problem:

$$
\frac{\mathrm{d} Q}{\mathrm{~d} z}=-2 \sqrt{Q\left(I_{\text {out }}+Q\right)}\left[\delta+m\left(I_{\text {out }}+2 Q\right)\right] \leq 0 .
$$

The linear operator $\mathcal{J} \mathcal{L}_{*}$ in $(5.2)$ can be explicitly written in the form (2.6), (2.25), and (2.26) with

$$
\begin{aligned}
& W_{1}=-2 m\left(I_{\text {out }}+2 Q\right)\left[\begin{array}{ll}
0 & 1 \\
1 & 0
\end{array}\right] \\
& W_{2}=-m I_{\text {out }}\left[\begin{array}{ll}
0 & 1 \\
1 & 0
\end{array}\right]-2 \mathrm{i} m \sqrt{Q\left(I_{\text {out }}+Q\right)}\left[\begin{array}{ll}
1 & 0 \\
0 & 1
\end{array}\right] .
\end{aligned}
$$

The eigenvalue problem $\left(\mathcal{J} \mathcal{L}_{*}-\lambda\right) \boldsymbol{\psi}(z)=0$ for $\boldsymbol{\psi}(z)=\left(\psi_{1}, \psi_{2}, \psi_{3}, \psi_{4}\right)^{T}$ decomposes into two eigenvalue problems for $\boldsymbol{\psi}_{ \pm}(z)$ :

$$
\boldsymbol{\psi}_{ \pm}(z)=\left[\begin{array}{c}
\psi_{1} \pm \psi_{3} \\
\psi_{2} \mp \psi_{4}
\end{array}\right] .
$$

The new uncoupled problems take the form

$$
\begin{aligned}
&-\mathrm{i} \frac{\mathrm{d} \psi_{ \pm 1}}{\mathrm{~d} z}-\left[\delta+2 m\left(I_{\text {out }}+2 Q\right)\right] \psi_{ \pm 2} \mp 2 \mathrm{i} m \sqrt{Q\left(I_{\text {out }}+Q\right)} \psi_{ \pm 1} \pm m I_{\text {out }} \psi_{ \pm 2}=\mathrm{i} \lambda \psi_{ \pm 1}, \\
& \mathrm{i} \frac{\mathrm{d} \psi_{ \pm 2}}{\mathrm{~d} z}-\left[\delta+2 m\left(I_{\text {out }}+2 Q\right)\right] \psi_{ \pm 1} \pm 2 \mathrm{i} m \sqrt{Q\left(I_{\text {out }}+Q\right)} \psi_{ \pm 2} \mp m I_{\text {out }} \psi_{ \pm 1}=\mathrm{i} \lambda \psi_{ \pm 2} .
\end{aligned}
$$

Transforming further by the $z$-dependent change of coordinates

$$
\boldsymbol{\psi}_{ \pm}(z)=\left[\begin{array}{c}
\mathrm{i} U_{ \pm} \\
V_{ \pm}
\end{array}\right] \mathrm{e}^{\mp 2 m \int_{0}^{z} \sqrt{Q(\zeta)\left(I_{\text {out }}+Q(\zeta)\right)} d \zeta}
$$

we find two uncoupled eigenvalue problems with real coefficients:

$$
\begin{aligned}
-\frac{\mathrm{d} U_{ \pm}}{\mathrm{d} z}+\left[\delta+2 m\left(I_{\text {out }}+2 Q\right) \mp m I_{\text {out }}\right] V_{ \pm} & =\lambda U_{ \pm}, \\
\frac{\mathrm{d} V_{ \pm}}{\mathrm{d} z}-\left[\delta+2 m\left(I_{\text {out }}+2 Q\right) \pm m I_{\text {out }}\right] U_{ \pm} & =\lambda V_{ \pm} .
\end{aligned}
$$

The boundary conditions (5.3) have transformed to $U_{ \pm}(0)=V_{ \pm}(L)=0$. Using the energy principle, we find the following two relations for the system (9.4)-(9.5)

$$
(\lambda-\bar{\lambda})\left(V_{ \pm} \bar{U}_{ \pm}+U_{ \pm} \bar{V}_{ \pm}\right)=\frac{\mathrm{d}}{\mathrm{d} z}\left(V_{ \pm} \bar{U}_{ \pm}-\bar{V}_{ \pm} U_{ \pm}\right) .
$$

and

$$
(\lambda+\bar{\lambda})\left(\left|U_{ \pm}\right|^{2}+\left|V_{ \pm}\right|^{2}\right)=-\frac{\mathrm{d}}{\mathrm{d} z}\left(\left|U_{ \pm}\right|^{2}-\left|V_{ \pm}\right|^{2}\right) \mp 2 m I_{\text {out }}\left(\bar{U}_{ \pm} V_{ \pm}+U_{ \pm} \bar{V}_{ \pm}\right) .
$$

If $\operatorname{Im} \lambda \neq 0$, then

$$
\int_{0}^{L}\left(\bar{V}_{ \pm} U_{ \pm}+V_{ \pm} \bar{U}_{ \pm}\right) d z=0
$$


and therefore

$$
(\lambda+\bar{\lambda}) \int_{0}^{L}\left(\left|U_{ \pm}\right|^{2}+\left|V_{ \pm}\right|^{2}\right) d z=-\left|V_{ \pm}\right|^{2}(0)-\left|U_{ \pm}\right|^{2}(L)<0 .
$$

The last inequality follows from the fact that $V_{ \pm}(0) \neq 0$ and $U_{ \pm}(L) \neq 0$, because the first-order initial-value problem with zero initial data has a unique zero solution for $U(z), V(z)$. Thus, if $\lambda \in \mathbb{C}$, then $\operatorname{Re}(\lambda)<0$.

If $\lambda \in \mathbb{R}$, then $U(z)$ and $V(z)$ are real. Consider

$$
\frac{\mathrm{d}}{\mathrm{d} z}\left(U_{ \pm} V_{ \pm}\right)=\left[\delta+2 m\left(I_{\text {out }}+2 Q\right) \mp m I_{\text {out }}\right] V_{ \pm}^{2}+\left[\delta+2 m\left(I_{\text {out }}+2 Q\right) \pm m I_{\text {out }}\right] U_{ \pm}^{2}>0 .
$$

The last inequality is valid for $\delta \geq 0$ and $m>0$. As a result, for any $\lambda \in \mathbb{R}$

$$
\int_{0}^{L} U_{ \pm} V_{ \pm} d z>0
$$

Real eigenvalues $\lambda \in \mathbb{R}$ appear when complex eigenvalues coalesce. At the bifurcation point, $\int_{0}^{L} U_{ \pm} V_{ \pm} d z=0$, see (9.6). All eigenvalues $\lambda$ are complex for $I_{\text {out }}=0$. Since the potential $W$ is a bounded perturbation of $\mathcal{L}$, all eigenvalues of $\mathcal{J} \mathcal{L}_{*}$ stay complex for $I_{\text {out }}>0$ because of the constraint (9.8).

Thus, the spectrum of the problem $(9.4)-(9.5)$ in $L^{2}([0, L])$ is located in the open left half-plane $\operatorname{Re}(\lambda)<0$. No zero eigenvalues exist, and therefore no turning points where $\mathcal{I}_{L}^{\prime}\left(I_{\text {out }}\right)=0$, see Lemma 5.1. Therefore, the stationary solution $\mathbf{A}_{*}(z)$ is unique for a given value $I_{\mathrm{in}}$.

Remark 9.2 For the case $m<0$ and $\delta+m I_{\text {out }}>0$, the sign of $\int_{0}^{L} U_{ \pm} V_{ \pm} d z$ is not definite. Therefore, real eigenvalues are generally allowed. However, since $\mathcal{I}_{L}^{\prime}\left(I_{\text {out }}\right)>0$ for $n=0$ and complex eigenvalues have $\operatorname{Re}(\lambda)<0$, all real eigenvalues stay in the left half-plane of $\lambda$ for any $0<I_{\text {out }}<\frac{\delta}{|m|}$. For the case $m<0$ and $\delta+m I_{\text {out }}<0$, the sign of $\int_{0}^{L} U_{ \pm} V_{ \pm} d z$ is definite again (it is negative) and the proof of Proposition 9.1 applies to this case, as well.

Proposition 9.3 In the semi-infinite domain $z \in[0, \infty)$, stationary localized solutions $\mathbf{A}_{*}(z)$ of (3.1) with boundary conditions (3.12) and with potential $W$ in (1.3) with $n=0$ are spectrally stable for any $\delta$ and $m$.

Proof. We formally follow the proof of Proposition 9.1 with $I_{\text {out }}=0$ and $L=\infty$. Note that the transformation $(9.3)$ maps $L^{2}(0, \infty)$ into itself since $Q(z)$ decays exponentially. The energy principle for the system (9.4)-(9.5) with $I_{\text {out }}=0$ for eigenfunctions of the point spectrum with the boundary condition $U_{ \pm}(0)=0$ and sufficient decay at infinity takes the form:

$$
(\lambda+\bar{\lambda}) \int_{0}^{\infty}\left(|U|^{2}+|V|^{2}\right) d z=-\left.\left(|U|^{2}-|V|^{2}\right)\right|_{z=0} ^{z=\infty}=-|V|^{2}(0)<0 .
$$

Thus, $\operatorname{Re}(\lambda)<0$ for eigenvalues of the point spectrum both for $\lambda \in \mathbb{C}$ and for $\lambda \in \mathbb{R}$. 
Remark 9.4 Proposition 9.3 follows also from convergence results in Proposition 8.2. Indeed, the stationary localized solutions $\mathbf{A}_{*}\left(z ; \infty, Q_{\infty}\right)$ in $z \in[0, \infty)$ cannot be unstable if all stationary solutions $\mathbf{A}_{*}\left(z ; L, I_{\text {out }}\right)$ in the bounded interval $z \in[0, L]$ are stable.

Case $m=0$ : The transmission function $I_{\text {in }}=\mathcal{I}_{L}\left(I_{\text {out }}\right)$ is shown on Fig. 2(a) for $\delta=0.25$ and $n=1$. It displays optical bistability such that four turning points exist, where $\mathcal{I}_{L}^{\prime}\left(I_{\text {out }}\right)=0$. Studying this particular example, we show numerically several general results on unstable real and complex eigenvalues in the spectrum of $\mathcal{J} \mathcal{L}_{*}$ :

(i) Stationary solutions $\mathbf{A}_{*}(z)$ at the lowest positive-slope branch of $I_{\text {in }}=\mathcal{I}_{L}\left(I_{\text {out }}\right)$ are spectrally stable, see Proposition 5.3.

(ii) Stationary solutions $\mathbf{A}_{*}(z)$ at the negative-slope branch of $I_{\text {in }}=\mathcal{I}_{L}\left(I_{\text {out }}\right)$ are spectrally unstable with a single real positive eigenvalue $\lambda$, which is predicted by the sign of $E_{L}(0)$, see Proposition 6.2.

(iii) The real positive eigenvalue $\lambda$ disappears at both the lower and upper turning points of the negative-slope branch of $I_{\text {in }}=\mathcal{I}_{L}\left(I_{\text {out }}\right)$, see Proposition 7.5 for $L=\infty$.

(iv) Stationary solutions $\mathbf{A}_{*}(z)$ at the upper positive-slope branches of $I_{\text {in }}=\mathcal{I}_{L}\left(I_{\text {out }}\right)$ have a pair of complex eigenvalues $\lambda$ with $\operatorname{Re}(\lambda)>0$. The pair of complex eigenvalues occur after a Hopf bifurcation, where time-periodic solutions are born.

(v) The pair of complex eigenvalues $\lambda$ is preserved on the upper negative-slope branches of the function $I_{\text {in }}=\mathcal{I}_{L}\left(I_{\text {out }}\right)$.

For the example with $\delta=0.25$ and $n=1$, the transmission function $I_{\text {in }}=\mathcal{I}_{L}\left(I_{\text {out }}\right)$ has only two branches with negative slope and only four turning points where $\mathcal{I}_{L}\left(I_{\text {out }}\right)=0$ (see Fig. $2(\mathrm{a}))$. The Evans function $E_{L}(\lambda)$ is computed numerically from the determinant (6.4) for different values of $I_{\text {out }}$. It is shown on Fig. 4(a) for real positive $\lambda$. The values $I_{\text {out }}=0.008$ and $I_{\text {out }}=0.09$ belong to the lowest and second positive-slope branches of the function $I_{\text {in }}=\mathcal{I}_{L}\left(I_{\text {out }}\right)$, respectively, while the value $I_{\text {out }}=0.05$ belongs to the first negative-slope branch of $I_{\text {in }}=\mathcal{I}_{L}\left(I_{\text {out }}\right)$. The Evans function $E_{L}(\lambda)$ has a single positive zero for $I_{\text {out }}=0.05$ and no positive zeros for the other two values of $I_{\text {out }}$. In the limit of large positive $\lambda, E_{L}(\lambda)>0$ according to Lemma 5.1.

The unstable (real positive) eigenvalue $\lambda$ is predicted by the negative sign of $E_{L}(0)$ according to Proposition 6.2. If $E_{L}^{\prime}(0)>0$, the unstable eigenvalue $\lambda$ is associated with the negativeslope branch of $I_{\text {in }}=\mathcal{I}_{L}\left(I_{\text {out }}\right)$ and it disappears at the turning point where $\mathcal{I}_{L}^{\prime}\left(I_{\text {out }}\right)=0$. We 
compute numerically $E_{L}(0)$ and $E_{L}^{\prime}(0)$ as a function of $I_{\text {out }}$ and plot them on Fig. 4(b). The figure shows that there are exactly four values of $I_{\text {out }}$, where $E_{L}(0)=0$, which match the turning points of the function $\mathcal{I}_{L}\left(I_{\text {out }}\right)$. At all four turning points, $E_{L}^{\prime}(0)>0$ (including the first point, which can be seen from the figure after zoom). Thus, the zero eigenvalue $\lambda=0$ is algebraically simple at the turning points for this example. The result is always valid for the case $L=\infty$, see Proposition 7.5. We are not able to compute analytically the sign of $E_{L}^{\prime}(0)$ which is given by $E_{L}^{\prime}(0)=\left(\phi_{0}, \boldsymbol{\psi}_{0}\right)_{X}$ as in (B.10) of Appendix B, where $\boldsymbol{\psi}_{0}(z)$ is defined in (5.4) and $\phi_{0}(z)$ is defined in (A.5) of Appendix A.

The function $E_{L}(\lambda)$ for $\lambda \in \mathbb{R}$ does not give information on existence of complex unstable eigenvalues $\lambda$ with $\operatorname{Re}(\lambda)>0$. In order to study complex eigenvalues $\lambda$, we integrate $E_{L}(\lambda)$ in a complex plane of $\lambda$ and compute numerically the winding number

$$
N=\frac{1}{2 \pi \mathrm{i}} \int_{C} \frac{E_{L}^{\prime}(\lambda) \mathrm{d} \lambda}{E_{L}(\lambda)}
$$

where the contour $C$ is designed to trace all unstable eigenvalues, e.g. $C$ is the boundary of the rectangle:

$$
D=\{\lambda \in \mathbb{C}: 0 \leq \operatorname{Re}(\lambda) \leq \Lambda, \quad-M \leq \operatorname{Im}(\lambda) \leq M\},
$$

where $\Lambda$ and $M$ are sufficiently large. The winding number $N$ gives the number of zeros of $E_{L}(\lambda)$, which coincides with the number of unstable real $\left(N_{r}\right)$ and complex $\left(2 N_{c}\right)$ eigenvalues such that $N=N_{r}+2 N_{c}$.

An individual computation of the complex values of $E_{L}(\lambda)$ along the contour $C$ is shown on Fig. 5(a) for $I_{\text {out }}=0.09$. The value $I_{\text {out }}$ belongs to the second positive-slope branch of the function $I_{\text {in }}=\mathcal{I}_{L}\left(I_{\text {out }}\right)$, when no real positive eigenvalues $\lambda$ exist (see Fig. 4(a)). However, there exists a single pair of complex eigenvalues with $\operatorname{Re}(\lambda)>0$, since the argument of $E_{L}(\lambda)$ is increased by $4 \pi$ after a complete loop, $N=2$.

We compute the number $N$ as a function of $I_{\text {out }}$ and display it on Fig. 5(b). The lowest positive-slope and negative-slope branches of $\mathcal{I}_{L}\left(I_{\text {out }}\right)$ have $N=0$ and $N=1$, respectively, according to the discussion above. The pair of complex eigenvalues with $N=2$ crosses the imaginary axis at $I_{\text {out }} \approx 0.065$ generating a Hopf bifurcation. The stationary solution is spectrally stable in a narrow region at the second positive-slope branch of $\mathcal{I}_{L}\left(I_{\text {out }}\right)$ between the second turning point at $I_{\text {out }} \approx 0.06$ and the Hopf bifurcation point at $I_{\text {out }} \approx 0.065$. The pair of complex eigenvalues is preserved for any stationary solution with $I_{\text {out }}>0.065$, including the solution at the second negative-slope branch of $\mathcal{I}_{L}\left(I_{\text {out }}\right)$, where $N=3$. A new pair of complex eigenvalues arises at $I_{\text {out }} \approx 0.950$ via a new Hopf bifurcation and stays for all $I_{\text {out }}>0.950$. 

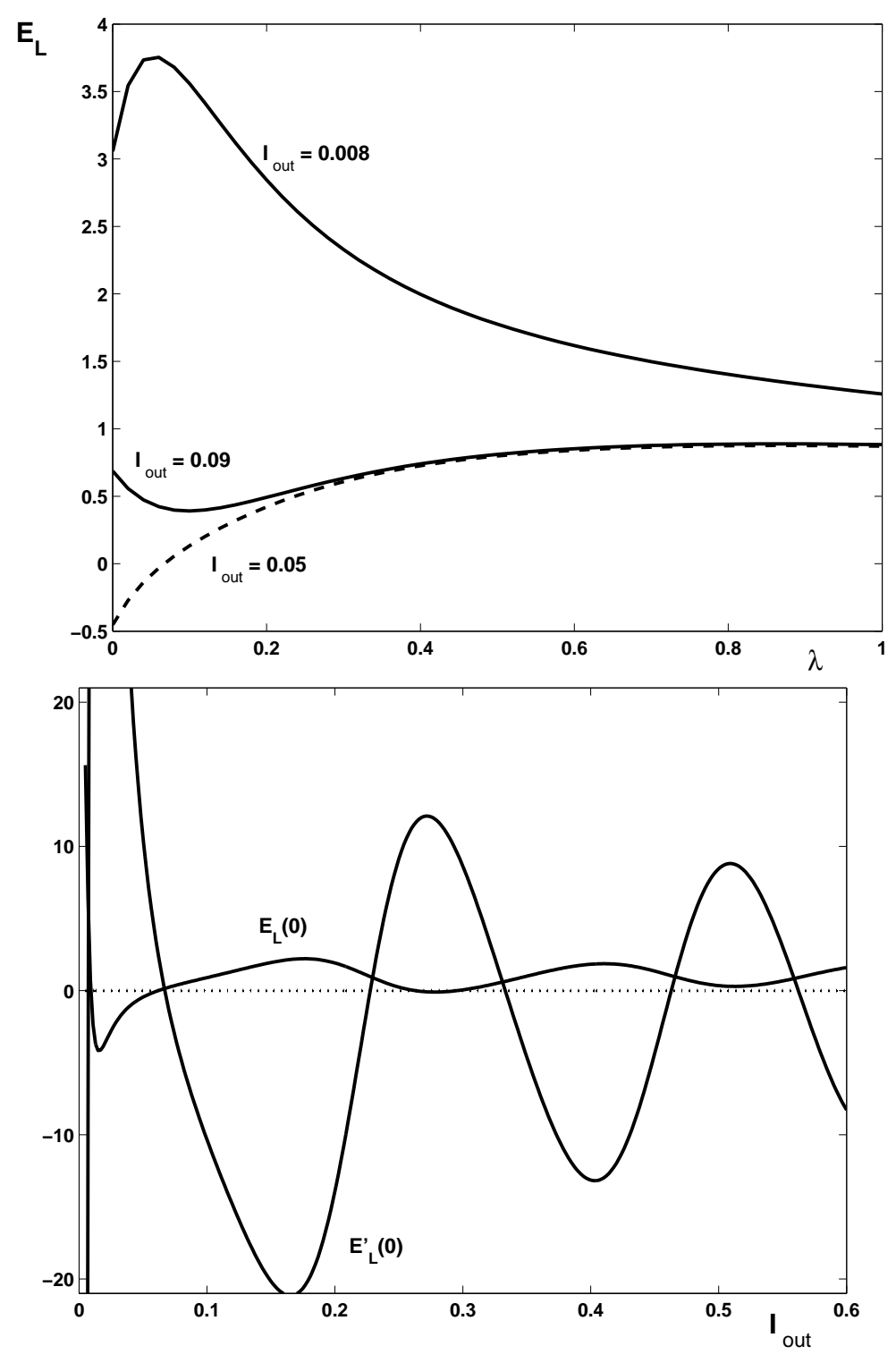

Figure 4: (a) The Evans function $E_{L}(\lambda)$ for real positive $\lambda$ at three values of $I_{\text {out }}$. Parameter values are provided in the text. There exists a positive zero of $E_{L}(\lambda)$ for $I_{\text {out }}=0.05$ (dotted curve). (b) The values $E_{L}(0)$ and $E_{L}^{\prime}(0)$ as functions of $I_{\text {out }}$. Zero eigenvalues at the turning points, where $E_{L}(0)=0$, are always algebraically simple since $E_{L}^{\prime}(0)>0$. 

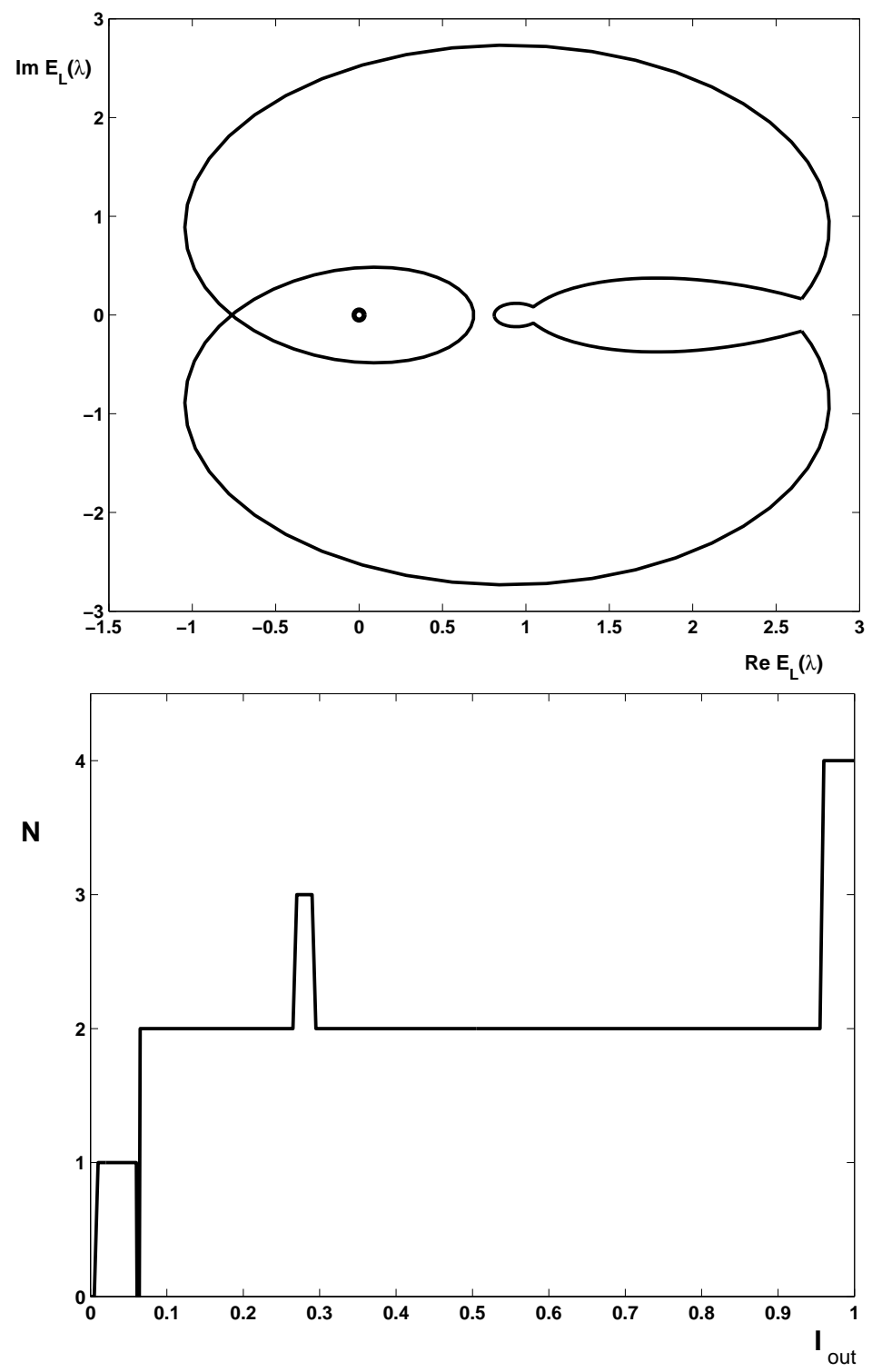

Figure 5: (a) The trace of $E_{L}(\lambda)$ along the contour $\lambda \in \mathbb{C}$. The argument of $E_{L}(\lambda)$ is increased by $4 \pi$ in a complete loop. (b) The winding number $N$ of the Evans function $E_{L}(\lambda)$ versus $I_{\text {out }}$. The pair of complex eigenvalues is preserved for any $I_{\text {out }}>0.065$. 


\section{Conclusion}

We have developed mathematical tools for the study of light transmission in the Bragg resonance gap of nonlinear photonic gratings. We therefore study a set of nonlinear coupled mode equations for the amplitudes of forward and backward propagating light. Focusing on existence and stability of stationary light transmission, we obtain spectral stability and instability results in finite, large, and semi-infinite structures. In all cases, a positive slope of the (inverse) transmission functions $I_{\text {in }}=\mathcal{I}_{L}\left(I_{\text {out }}\right)$ and $I_{\text {in }}=\mathcal{I}_{\infty}\left(I_{\text {out }}\right)$, is necessary for a stable light transmission.

We show that in finite structures, spectral stability implies nonlinear asymptotic stability. The system, although conservative in the absence of boundary conditions, effectively behaves like a dissipative dynamical system, due to radiation loss through the boundaries. The main technical difficulty is to show that the radiation loss actually implies smoothing after finite time, similar to the smoothing properties of delay equations. This smoothing property allows us to overcome regularity problems at the boundary.

Instabilities of stationary light transmission typically occur at fold points of the transmission function or when a pair of complex eigenvalues crosses the imaginary axis. Both bifurcations can be analyzed on one- and two-dimensional smooth center-manifolds, respectively, and the reduced dynamics show the typical exchange of stability for these bifurcations. As a main technical tool for actual spectral computations, we make use of the Evans function. We relate the instability criterion $E_{L}(0)<0$ to the more classical Leray-Schauder degree of the linearization. We use numerical computations of winding numbers of $E_{L}(\lambda)$ to accurately determine the number of complex unstable eigenvalues.

In semi-infinite structures, finite-dimensional reductions and degree arguments would have to be much more delicate. We focus on spectral stability, using again the Evans function $E_{\infty}(\lambda)$. We show that factoring the exponential decay of the output intensity, we can define a renormalized transmission function $I_{\text {in }}=\mathcal{I}_{\infty}\left(Q_{\infty}\right)$. The sign of $\mathcal{I}_{\infty}^{\prime}\left(Q_{\infty}\right)$ coincides with the sign of $E_{\infty}(0)$ and with the negative slope $Q^{\prime}(0)$ of the intensity of the stationary solution at the boundary $z=0$. In particular, at fold points of the transmission function, the solution branches exchange their stability, while the amplitude of the stationary solution reaches its maximal value.

We also investigate the limit when the length of the structure tends to infinity. In this limit, we show that the bifurcation diagram for stationary solutions and spectra of the linearization at stationary solutions converge. The (neutrally stable) essential spectrum is approximated by dense clusters of eigenvalues in large structures. We compute the asymptotic location 
of these clusters depending on the zeroes of a scattering polynomial, which measures the strength of the reflection and transmission of linear waves at the nonlinear structure. For small incident intensities, the clusters are located in the stable complex plane, such that small intensity light transmission is stable, for all sizes of the structure. Bogdanov-Takens points, potentially generating more complicated dynamics, can be excluded in semi-infinite and in large structures.

For a specific cubic nonlinearity, we have considered examples of all-optical limiting and optical bistability. For all-optical limiting, we show that the (inverse) transmission function $I_{\text {in }}=$ $\mathcal{I}_{L}\left(I_{\text {out }}\right)$ has positive slope for all intensities and all stationary solutions are asymptotically stable. For optical bistability, we show that the instabilities of negative-slope stationary solutions are complicated due to Hopf bifurcations which destabilize stationary solutions at upper positive-slope branches.

All of our spectral instability results imply nonlinear instability. We show that in finite structures, spectral stability implies nonlinear asymptotic stability. We did not address asymptotic stability in semi-infinite structures or asymptotic stability in finite-length structures, with uniform bounds on convergence rates or on the basin of attraction in large structures.

In summary, we have shown how spatial dynamics and Evans function methods can serve as tools in the analysis and numerics of bifurcation scenarios. As compared to functional-analytic methods, they allow a smooth passage between finite, large, and semi-infinite structures. Even with an ad hoc implementation, numerical computations of the Evans function, following the spirit of the analysis, seem to provide more reliable results than direct finite-difference approximations.

\section{Appendix A: The adjoint operator of $\mathcal{J} \mathcal{L}_{*}$ and its kernel}

We consider eigenvectors $\psi(z)$ of the operator $\mathcal{J} \mathcal{L}_{*}$, see (5.2) and (5.3), and eigenvectors $\phi(z)$ of the adjoint operator $\left(\mathcal{J} \mathcal{L}_{*}\right)^{a d}=-\mathcal{L}_{*} \mathcal{J}$ with adjoint boundary conditions. Since we merely discuss ordinary differential equations rather than abstract operators on function spaces, we will refer to the operators $\mathcal{J} \mathcal{L}_{*},\left(\mathcal{J} \mathcal{L}_{*}\right)^{\text {ad }}$ in a pointwise sense, incorporating the boundary conditions in a second stage. In particular, by an eigenvector, we understand a solution of the differential equation associated with $\mathcal{J L}_{*} \boldsymbol{\psi}(z)=\lambda \boldsymbol{\psi}(z)$, without any restriction on the boundary values.

Recall that we equipped the phase space $X=\left[L^{2}(0, L)\right]^{4}$ with the standard scalar product

$$
(\phi, \psi)_{X}=\int_{0}^{L}\left(\bar{\phi}_{1} \psi_{1}+\bar{\phi}_{2} \psi_{2}+\bar{\phi}_{3} \psi_{3}+\bar{\phi}_{4} \psi_{4}\right) \mathrm{d} z
$$


The eigenvector $\phi(z)$ to the eigenvalue $\bar{\lambda}$ solves the linear system of differential equations

$$
-\mathcal{L}_{*} \mathcal{J} \phi=\bar{\lambda} \phi
$$

with the boundary conditions

$$
\phi_{2}(0)=\phi_{4}(0)=0, \quad \phi_{1}(L)=\phi_{3}(L)=0
$$

Denote by

$$
P[\boldsymbol{\phi}, \boldsymbol{\psi}]:=\mathrm{i} \omega(\boldsymbol{\phi}, \boldsymbol{\psi})=\mathrm{i}(\boldsymbol{\phi}, j \boldsymbol{\psi})=-\bar{\phi}_{1} \psi_{1}+\bar{\phi}_{2} \psi_{2}-\bar{\phi}_{3} \psi_{3}+\bar{\phi}_{4} \psi_{4},
$$

a scalar multiple of the symplectic two-form, introduced in (3.4). A short computation shows that this two-form is independent of $z$ when evaluated on eigenvectors $\psi(z)$ to an eigenvalue $\lambda$ and the adjoint eigenvectors to $\bar{\lambda}$

$$
\frac{\mathrm{d}}{\mathrm{d} z} P[\phi, \boldsymbol{\psi}]=0
$$

Let $\mathbf{A}_{*}\left(z ; I_{\mathrm{pt}}, h\right)$ be a stationary solution of (3.1), parameterized by the Hamiltonian $h$ in (3.3) and the additional conserved quantity $I_{\mathrm{pt}}$ in (3.6). The stationary solution $\mathbf{A}_{*}(z) \equiv$ $\mathbf{A}_{*}\left(z ; I_{\text {out }}, h_{s}\left(I_{\text {out }}\right)\right)$ satisfies the boundary conditions $(3.2)$. We show that, if $\mathcal{I}_{L}^{\prime}\left(I_{\text {out }}\right)=0$, the eigenvector $\phi_{0}(z)$ in the kernel of $\mathcal{L}_{*} \mathcal{J}$ is given by

$$
\phi_{0}(z)=\mathcal{J}\left[\left.Q^{\prime}(0) \frac{\partial \mathbf{A}_{*}}{\partial h}\left(z ; I_{\text {out }}, h\right)\right|_{h=h_{s}\left(I_{\text {out }}\right)}-\left.\frac{\partial Q(0)}{\partial h}\right|_{h=h_{s}\left(I_{\text {out }}\right)}\left(\mathbf{A}_{*}^{\prime}(z)-\theta^{\prime}(L) \mathcal{J} \mathbf{A}_{*}(z)\right)\right] .
$$

We therefore derive relations between eigenfunctions in the kernel of $\mathcal{L}_{*}$. We define four fundamental eigenvectors $\boldsymbol{\psi}_{1,2,3,4}(z)$ in the kernel of $\mathcal{L}_{*}$ as derivatives of the family of stationary solutions $\mathbf{A}_{*}\left(z ; I_{\mathrm{pt}}, h\right)$ with respect to the parameters

$$
\begin{gathered}
\boldsymbol{\psi}_{1}=\left.\frac{\partial \mathbf{A}_{*}}{\partial I_{\text {out }}}\left(z ; I_{\text {out }}, h\right)\right|_{h=h_{s}\left(I_{\text {out }}\right)}, \quad \boldsymbol{\psi}_{2}=\mathcal{J} \mathbf{A}_{*}(z), \\
\boldsymbol{\psi}_{3}=\mathbf{A}_{*}^{\prime}(z), \quad \boldsymbol{\psi}_{4}=\left.\frac{\partial \mathbf{A}_{*}}{\partial h}\left(z ; I_{\text {out }}, h\right)\right|_{h=h_{s}\left(I_{\text {out }}\right)} .
\end{gathered}
$$

The four corresponding eigenvectors of the adjoint operator are $\phi_{j}(z)=\mathcal{J} \boldsymbol{\psi}_{j}(z)$. We construct the matrix elements $P_{i, j}$ from the two-forms $P\left[\boldsymbol{\phi}_{i}, \boldsymbol{\psi}_{j}\right]$ between these eigenvectors:

$$
P_{i, j}=P\left[\mathcal{J} \boldsymbol{\psi}_{i}, \boldsymbol{\psi}_{j}\right]=\mathrm{i}\left[\bar{\psi}_{i 1} \psi_{j 1}-\bar{\psi}_{i 2} \psi_{j 2}-\bar{\psi}_{i 3} \psi_{j 3}+\bar{\psi}_{i 4} \psi_{j 4}\right]
$$

The matrix elements $P_{i, j}$ are constant in $z$ but non-zero in general, since the eigenvectors $\boldsymbol{\psi}_{j}(z)$ do not satisfy the boundary conditions (5.3) and (A.3). The matrix elements $P_{i, j}$ can be computed explicitly from (3.5), (3.6), and (3.9): 


$$
\begin{array}{llll}
P_{1,1}=0, & P_{1,2}=-1, & P_{1,3}=-h_{\mathrm{s}}^{\prime}\left(I_{\text {out }}\right), & P_{1,4}=\alpha \\
P_{2,1}=1, & P_{2,2}=0, & P_{2,3}=0, & P_{2,4}=0 \\
P_{3,1}=h_{\mathrm{s}}^{\prime}\left(I_{\text {out }}\right), & P_{3,2}=0, & P_{3,3}=0, & P_{3,4}=1 \\
P_{4,1}=-\alpha, & P_{4,2}=0, & P_{4,3}=-1, & P_{4,4}=0,
\end{array}
$$

where

$$
\alpha=\mathrm{i}\left(\left.\frac{\partial \bar{a}}{\partial I_{\text {out }}} \frac{\partial a}{\partial h}\right|_{h=h_{s}}-\left.\frac{\partial \bar{b}}{\partial I_{\text {out }}} \frac{\partial b}{\partial h}\right|_{h=h_{s}}-\left.\frac{\partial a}{\partial I_{\text {out }}} \frac{\partial \bar{a}}{\partial h}\right|_{h=h_{s}}+\left.\frac{\partial b}{\partial I_{\text {out }}} \frac{\partial \bar{b}}{\partial h}\right|_{h=h_{s}}\right) .
$$

We parameterize the stationary solutions (3.10) through the boundary values: $Q(L)=0$ for $h=h_{s}\left(I_{\text {out }}\right)$ and $\theta(L)=0$ for any $h$. Since $\boldsymbol{\psi}_{3}(z)$ and $\boldsymbol{\psi}_{4}(z)$ are non-singular at $z=L$, we have

$$
\lim _{z \rightarrow L-0} \frac{1}{\sqrt{Q(z)}} Q^{\prime}(z)=\gamma_{1}<\infty,\left.\quad \lim _{z \rightarrow L-0} \frac{1}{\sqrt{Q(z)}} \frac{\partial Q(z)}{\partial h}\right|_{h=h_{s}\left(I_{\text {out }}\right)}=\gamma_{2}<\infty
$$

and, therefore,

$$
Q^{\prime}(L)=0,\left.\quad \frac{\partial Q(L)}{\partial h}\right|_{h=h_{s}\left(I_{\text {out }}\right)}=0 .
$$

The matrix elements $P_{i, j}$ are constants in $z$ and can be computed separately at $z=0$ and $z=L$. We evaluate $P_{i, j}$ at the right end $z=L$ and derive a set of relations for the parameters of stationary solutions $\mathbf{A}_{*}(z)$ :

$$
\begin{gathered}
P_{1,3}=-\theta^{\prime}(L)=-h_{\mathrm{s}}^{\prime}\left(I_{\text {out }}\right), \\
P_{1,4}=0=\alpha, \\
P_{3,4}=\left.\lim _{z \rightarrow L^{-}} Q^{\prime}(z) \frac{\partial \phi(z)}{\partial h}\right|_{h=h_{s}\left(I_{\text {out }}\right)}=1 .
\end{gathered}
$$

When we evaluate $P_{i, j}$ at the left boundary, $z=0$, we find another set of relations for the parameters of $\mathbf{A}_{*}(z)$ :

$$
\begin{gathered}
P_{1,3}=-\mathcal{I}^{\prime}\left(I_{\text {out }}\right)\left[\theta^{\prime}(0)-\phi^{\prime}(0)\right]-\phi^{\prime}(0)+Q^{\prime}(0) \frac{\partial}{\partial I_{\text {out }}}[\theta(0)-\phi(0)]=-h_{\mathrm{s}}^{\prime}\left(I_{\text {out }}\right), \\
P_{1,4}=-\left.\mathcal{I}^{\prime}\left(I_{\text {out }}\right) \frac{\partial}{\partial h}[\theta(0)-\phi(0)]\right|_{h=h_{s}}-\left.\frac{\partial \phi(0)}{\partial h}\right|_{h=h_{s}}+\left.\frac{\partial Q(0)}{\partial h}\right|_{h=h_{s}} \frac{\partial}{\partial I_{\text {out }}}[\theta(0)-\phi(0)]=0 \\
P_{3,4}=-\left.Q^{\prime}(0) \frac{\partial}{\partial h}[\theta(0)-\phi(0)]\right|_{h=h_{s}}+\left.\frac{\partial Q(0)}{\partial h}\right|_{h=h_{s}}\left[\theta^{\prime}(0)-\phi^{\prime}(0)\right]=1
\end{gathered}
$$

Now consider the eigenfunctions $\boldsymbol{\psi}_{3}(z)-\theta^{\prime}(L) \boldsymbol{\psi}_{2}(z)$ and $\boldsymbol{\psi}_{4}(z)$. These eigenfunctions are linearly independent and satisfy the right boundary conditions $\phi_{1}(L)=\phi_{3}(L)=0$ because of (A.10) and (A.11). A general solution to (A.2) with $\lambda=0$ satisfying the right boundary conditions is $\mathcal{J} \phi(z)=c_{1}\left[\boldsymbol{\psi}_{3}(z)-\theta^{\prime}(L) \boldsymbol{\psi}_{s}(z)\right]+c_{2} \boldsymbol{\psi}_{4}(z)$. The general solution satisfies the 
left boundary conditions $\phi_{2}(0)=\phi_{4}(0)=0$ when the determinant $\mathcal{D}$ of a linear system for $c_{1}$ and $c_{2}$ is zero. The determinant of this linear system is:

$$
\mathcal{D}=\mathrm{i}\left[\left.\frac{\partial Q(0)}{\partial h}\right|_{h=h_{s}}\left[\phi^{\prime}(0)-\theta^{\prime}(L)\right]-\left.Q^{\prime}(0) \frac{\partial \phi(0)}{\partial h}\right|_{h=h_{s}}\right] .
$$

By virtue of relations (A.11), (A.14), (A.15), and (A.16), we verify that $\mathcal{D}=-\mathrm{i} \mathcal{I}_{L}^{\prime}\left(I_{\text {out }}\right)$. When $\mathcal{I}_{L}^{\prime}\left(I_{\text {out }}\right)=0$, the rank of the coefficient matrix for $c_{1}$ and $c_{2}$ is one, since $Q^{\prime}(0)$ and $\left.\frac{\partial Q(0)}{\partial h}\right|_{h=h_{s}}$ may not vanish simultaneously, see (A.16). The eigenvector $\phi_{0}(z)$ is then given by (A.5).

\section{Appendix B: Derivative formulas for the Evans functions $E_{L}(\lambda)$ and $E_{\infty}(\lambda)$}

If $\lambda=\lambda_{0}$ is an eigenvalue of $\mathcal{J} \mathcal{L}_{*}$, the Evans function $E_{L}(\lambda)$ has the Taylor expansion at $\lambda=\lambda_{0}$ :

$$
E_{L}(\lambda)=c_{m}\left(\lambda-\lambda_{0}\right)^{m}+\mathrm{O}\left(\lambda-\lambda_{0}\right)^{m+1},
$$

where $m$ is the algebraic multiplicity of $\lambda_{0}$. In order to determine if the eigenvalue is algebraically simple, we therefore need to determine whether $E_{L}^{\prime}\left(\lambda_{0}\right)$ vanishes. Since eigenvalues are geometrically simple in our case, we may use Fredholm's alternative and find that $E_{L}^{\prime}\left(\lambda_{0}\right)$ vanishes precisely when the scalar product between nontrivial elements in the kernel and the kernel of the adjoint banishes. Alternatively, we can actually compute the derivative of the determinant $E_{L}(\lambda)$. The following lemma states an explicit formula for this derivative after an appropriate normalization.

Lemma B.1 Let $\lambda=\lambda_{0}$ be an eigenvalue of $\mathcal{J} \mathcal{L}_{*}$ in the case $L<\infty$ with a single eigenvector $\hat{\boldsymbol{\psi}}_{0}(z)$, normalized by $\hat{\psi}_{01}(L)=u_{23}^{+}(0 ; \lambda)$. Let $\hat{\boldsymbol{\phi}}_{0}(z)$ be the eigenvector of $\left(\mathcal{J} \mathcal{L}_{*}\right)^{\text {ad }}$, normalized by $\hat{\phi}_{01}(0)=\mathrm{e}^{-2 \bar{\lambda}_{0} L}$. Then,

$$
E_{L}^{\prime}\left(\lambda_{0}\right)=\left(\hat{\phi}_{0}, \hat{\psi}_{0}\right)_{X}
$$

Proof. Let the function $\boldsymbol{\psi}_{\lambda}(z)$ be defined by the following linear combination for any $\lambda \in \mathbb{C}$ :

$$
\boldsymbol{\psi}_{\lambda}(z)=u_{23}^{+}(0 ; \lambda) \mathbf{u}_{1}^{+}(z ; \lambda)-u_{13}^{+}(0 ; \lambda) \mathbf{u}_{2}^{+}(z ; \lambda) .
$$

It follows from (6.2) and (6.4) that the eigenfunction $\boldsymbol{\psi}_{\lambda}(z)$ satisfies the boundary conditions (5.3), except for the first component:

$$
\psi_{\lambda 1}(0)=\left|\begin{array}{cc}
u_{11}^{+}(0 ; \lambda) & u_{22}^{+}(0 ; \lambda) \\
u_{13}^{+}(0 ; \lambda) & u_{23}^{+}(0 ; \lambda)
\end{array}\right|=E_{L}(\lambda) \mathrm{e}^{2 \lambda L} .
$$


It follows from (B.3) and (B.4) that $\boldsymbol{\psi}_{\lambda}(z)=\hat{\boldsymbol{\psi}}_{0}(z)$ at $\lambda=\lambda_{0}$. By direct computations from the eigenvalue problems for $\hat{\boldsymbol{\psi}}_{0}(z)$ and $\hat{\boldsymbol{\psi}}_{0}(z)$, we verify that

$$
\left(\hat{\boldsymbol{\phi}}_{0}, \hat{\boldsymbol{\psi}}_{0}\right)_{X}=\left.P\left[\hat{\boldsymbol{\phi}}_{0},\left.\frac{\partial \boldsymbol{\psi}_{\lambda}}{\partial \lambda}\right|_{\lambda=\lambda_{0}}\right]\right|_{z=0} ^{z=L}=\overline{\hat{\phi}}_{01}(0) E_{L}^{\prime}\left(\lambda_{0}\right) \mathrm{e}^{2 \lambda_{0} L}=E_{L}^{\prime}(\lambda)
$$

Lemma B.2 Let $\lambda=\lambda_{0}$ be an eigenvalue of $\mathcal{J} \mathcal{L}_{*}$ in the case $L=\infty$ with a single eigenvector $\hat{\boldsymbol{\psi}}_{0}(z)$, normalized by $\lim _{z \rightarrow \infty} \mathrm{e}^{\nu z} \psi_{01}(z)=u_{32}^{+}(0 ; \lambda)$, where $\nu=\sqrt{\delta^{2}+\lambda_{0}^{2}}$ such that $\operatorname{Re}(\nu)>0$. Let $\hat{\phi}_{0}(z)$ be the eigenvector of $\left(\mathcal{J} \mathcal{L}_{*}\right)$ ad , normalized by $\hat{\phi}_{01}(0)=1$. Then,

$$
E_{\infty}^{\prime}\left(\lambda_{0}\right)=\left(\hat{\phi}_{0}, \hat{\psi}_{0}\right)_{X}
$$

Proof. Define a function $\boldsymbol{\psi}_{\lambda}(z)$ by the linear combination for any $\lambda \in \mathbb{C}$ :

$$
\boldsymbol{\psi}_{\lambda}(z)=u_{32}^{+}(0 ; \lambda) \mathbf{u}_{1}^{+}(z ; \lambda)-u_{32}^{+}(0 ; \lambda) \mathbf{u}_{2}^{+}(z ; \lambda) \text {. }
$$

This function is localized as $z \rightarrow+\infty$, satisfying $\psi_{\lambda 3}(0)=0$ and also $\psi_{\lambda 1}(0)=E_{\infty}(0)$. It follows from (B.7) that $\boldsymbol{\psi}_{\lambda}(z)=\hat{\boldsymbol{\psi}}_{0}(z)$ at $\lambda=\lambda_{0}$. Using direct computations, we verify again that

$$
\left(\hat{\boldsymbol{\phi}}_{0}, \hat{\boldsymbol{\psi}}_{0}\right)_{X}=\left.P\left[\hat{\boldsymbol{\phi}}_{0},\left.\frac{\partial \boldsymbol{\psi}_{\lambda}}{\partial \lambda}\right|_{\lambda=\lambda_{0}}\right]\right|_{z=0} ^{z=\infty}=\hat{\bar{\phi}}_{01}(0) E_{\infty}^{\prime}\left(\lambda_{0}\right)=E_{\infty}^{\prime}(0) .
$$

Below we apply these derivative formulas to actual computations of $E_{L}^{\prime}(0)$ and $E_{\infty}^{\prime}(0)$ when the kernel of $\mathcal{J} \mathcal{L}_{*}$ is non-empty. For the case $L<\infty$, when $\mathcal{I}_{L}^{\prime}\left(I_{\text {out }}\right)=0$, we use (5.4), (6.6) and (A.5), (A.11)-(A.16) of Appendix A to find the normalized eigenvectors in the form

$$
\hat{\boldsymbol{\psi}}_{0}=\bar{a}(0) \boldsymbol{\psi}_{0}(z), \quad \hat{\phi}_{0}=\frac{1}{a(0)} \phi_{0}(z)
$$

such that $\hat{\psi}_{01}(L)=u_{23}^{+}(0 ; \lambda)$ and $\hat{\phi}_{01}(0)=1$, where $a(z)$ is the first component of the stationary solution $\mathbf{A}_{*}(z)$. As a result, $E_{L}^{\prime}(0)=\left(\hat{\phi}_{0}, \hat{\boldsymbol{\psi}}_{0}\right)_{X}=\left(\phi_{0}, \boldsymbol{\psi}_{0}\right)_{X}$. Using parameterization (3.10), we may explicit this formula as

$$
\begin{gathered}
E_{L}^{\prime}(0)=2 \int_{0}^{L} d z\left[\frac{\partial Q(z)}{\partial h} \frac{\partial \phi(z)}{\partial I_{\text {out }}} Q^{\prime}(0)-\frac{\partial Q(z)}{\partial I_{\text {out }}} \frac{\partial \phi(z)}{\partial h} Q^{\prime}(0)\right. \\
+\frac{\partial Q(z)}{\partial I_{\text {out }}} \phi^{\prime}(z) \frac{\partial Q(0)}{\partial h}-Q^{\prime}(z) \frac{\partial \phi(z)}{\partial I_{\text {out }}} \frac{\partial Q(0)}{\partial h} \\
\left.+Q^{\prime}(z) \frac{\partial Q(0)}{\partial h} \frac{\partial \theta(0)}{\partial I_{\text {out }}}-\frac{\partial Q(z)}{\partial h} Q^{\prime}(0) \frac{\partial \theta(0)}{\partial I_{\text {out }}}-\frac{\partial Q(z)}{\partial I_{\text {out }}} \frac{\partial Q(0)}{\partial h} \theta^{\prime}(L)\right] .
\end{gathered}
$$


It seems difficult to draw any conclusion on the sign of $E_{L}^{\prime}(0)$ from (B.10). However, in the case $L=\infty$ the sign of $E_{\infty}^{\prime}(0)$ is strictly positive. For the case $L=\infty$, when $Q^{\prime}(0)=0$, we define the normalized eigenvectors in the form:

$$
\hat{\boldsymbol{\psi}}_{0}(z)=-\frac{\bar{a}(0)}{2 \delta Q_{\infty}} \boldsymbol{\psi}_{0}(z), \quad \hat{\phi}_{0}(z)=-\frac{1}{2 a(0) \theta^{\prime}(0)} \phi_{0}(z),
$$

such that $\lim _{z \rightarrow \infty} \mathrm{e}^{\delta z} \hat{\psi}_{01}(z)=u_{32}^{+}(0 ; 0)$ and $\hat{\phi}_{01}(0)=1$. As a result,

$$
E_{\infty}^{\prime}(0)=\left(\hat{\boldsymbol{\phi}}_{0}, \hat{\boldsymbol{\psi}}_{0}\right)_{X}=\frac{I_{\mathrm{in}}}{\delta Q_{\infty}}(>0)
$$

\section{References}

[1] W. Arendt et al. One-Parameter Semigroups of Positive Operators. Lecture Notes in Mathematics 1184, Springer-Verlag, Berlin, 1986.

[2] K. Deimling. Nichtlineare Gleichungen und Abbildungsgrade. Springer-Verlag, Berlin-New York, 1974.

[3] R. Gardner and C. K. R. T. Jones. A stability index for steady state solutions of boundary value problems for parabolic systems. J. Differential Equations 91 (1991), 181-203.

[4] R.A. Gardner and K. Zumbrun. The gap lemma and geometric criteria for instability of viscous shock profiles. Comm. Pure Appl. Math. 51 (1998), 797-855.

[5] H.M. Gibbs, Optical Bistability: Controlling Light with Light (Academic Press, New York, 1985).

[6] R.H. Goodman, M.I. Weinstein, and P.J. Holmes, "Nonlinear propagation of light in one-dimensional periodic structures", J. Nonlin. Science 11 (2001), 123-168.

[7] T. Kapitula and B. Sandstede. Stability of bright solitary wave solutions to perturbed nonlinear Schrödinger equations. Physica D 124 (1998), 58-103.

[8] T. Kato, Quasi-linear equations of evolution, with applications to partial differential equations, in Spectral theory and differential equations (Proc. Sympos., Dundee, 1974), pp. 25-70. Lecture Notes in Math. 448, Springer, Berlin, 1975.

[9] C. Kittel, "Introduction to solid-state physics" (John Wiley \& Sons, 1996).

[10] Yu.N. Ovchinnikov, "Stability problem in nonlinear wave propagation", JETP 87, 807 (1998). 
[11] Yu.N. Ovchinnikov and I.M. Sigal, "Optical bistability", JETP 93, 1004-1016 (2001).

[12] A. Pazy. Semigroups of linear operators and applications to partial differential equations. Applied Mathematical Sciences, 44. Springer-Verlag, New York, 1983.

[13] R.L. Pego and M.I. Weinstein, Convective linear stability of solitary waves for Boussinesq equations. Stud. Appl. Math. 99 (1997), 311-375.

[14] D. Pelinovsky and E.H. Sargent, "Stable all-optical limiting in nonlinear periodic structures. II: Computations", J. Opt. Soc. Am. B 19, 1873-1889 (2002).

[15] D. Pelinovsky, J. Sears, L. Brzozowski, and E.H. Sargent, "Stable all-optical limiting in nonlinear periodic structures. I: Analysis", J. Opt. Soc. Am. B 19, 43-53 (2002).

[16] B. Sandstede and A. Scheel, "On the stability of periodic travelling waves with large spatial period", J. Diff. Eqns. 172 (2001), 134-188.

[17] B. Sandstede and A. Scheel, "Absolute and convective instabilities of waves on unbounded and large bounded domains", Physica D 145, 233-277 (2000).

[18] M. Shub. Global stability of dynamical systems. Springer-Verlag, New York, 1987.

[19] C.M. de Sterke, "Stability analysis of nonlinear periodic media", Phys. Rev. A 45, 8252 (1992).

[20] C.M. de Sterke and J.E. Sipe, "Gap solitons", Progress in Optics, 33, 203 (1994). 